\title{
Land Condition Trend Analysis Avian Database: Ecological Guild-based Summaries
}

by

Eric R. Schreiber and William R. Whitworth

\begin{tabular}{|l|l|}
\hline Common Name & Barred Owl \\
\hline Scientific Name & Strix varia \\
\hline LCTA Code & STVA \\
\hline Alpha Code & BAOW \\
\hline AOU\# & 3680 \\
\hline $\begin{array}{l}\text { Neotropical } \\
\text { Migrant Status }\end{array}$ & Resident \\
\hline General Habitat & Forest, Swamp/Marsh \\
\hline Nest Type & Cavity \\
\hline Nest Location & Snag, Woody Upper Canopy \\
\hline Food Type & Small Mammals, Birds \\
\hline $\begin{array}{l}\text { Foraging } \\
\text { Technique }\end{array}$ & Low Patrol, Hover \& Pounce \\
\hline
\end{tabular}

Land Condition Trend Analysis (LCTA) bird database documentation capabilities often are limited to generating installation-wide species checklists, estimates of relative abundance, and evidence of breeding activity. The conservation and characterization of biological diversity is becoming a management objective of both government and private organizations, and as such, more ecologically descriptive summaries are being required.

Ecological guild analyses have been proposed as a means to link wildlife to a specific biodiversity component, and thus represent an option to military trainers in evaluating impacts to wildlife communities.

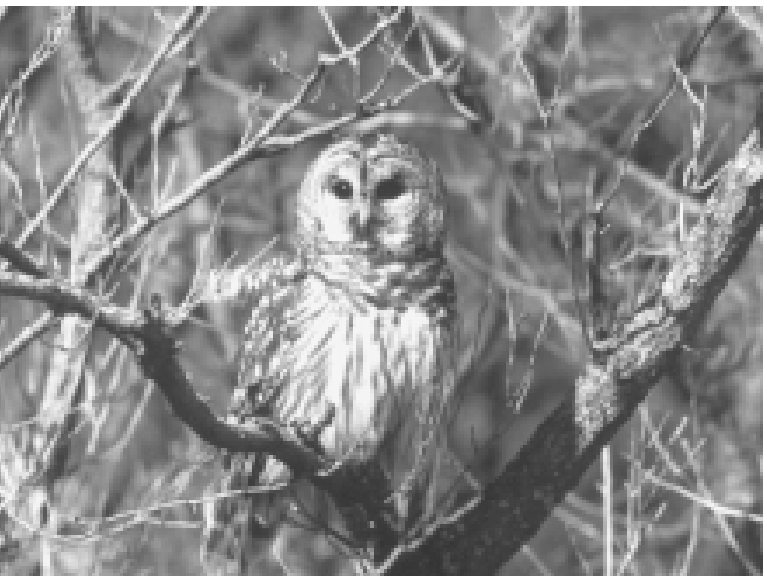

This report documents an LCTA database update that provides selected ecological attributes (particularly for the nesting season) for 676 bird species occurring within the continental United States. This database upgrade provides land managers the capability to: (1) generate ecological guild-based summaries with no additional fieldwork, (2) identify specific groups of birds most likely to be impacted by a spatially discrete training impact, (3) customize the local database by adjusting individual attributes for species specific to the installation, and (4) conveniently cross reference or simply convert all LCTA species codes to more globally recognized American Ornithological Union "AOU numbers" or the Bird Banding Laboratories "Alpha codes." 


\section{Foreword}

This study was conducted for the Office of the Directorate of Environmental Programs (ODEP), Assistant Chief of Staff for Installation Management (ACSIM) under Project 4A162720A896, "Environmental Quality Technology"; Work Unit EN-UD5, "LCTA Applications for Integrated Natural Resources Management and Environmental Compliance." The technical monitor was Dr. Victor E. Diersing, DAIM-ED-N.

The work was performed by the Natural Resources and Management Division (LL-N) of the Land Management Laboratory (LL), U.S. Army Construction Engineering Research Laboratories (USACERL). Mr. Schreiber was employed as a Research Assistant under an interagency agreement with the U.S. Forest Service, Rocky Mountain Range and Forest Experiment Station, and Colorado State University. Special thanks are given to Alan Anderson, USACERL researcher, for developing the bird macro used in conjunction with this report. The authors thank and give credit to Samuel Orr, Fort Sill, Oklahoma, for the Barred Owl cover photo. The USACERL Principal Investigator was Dr. David L. Price. Dr. J ohn T. Bandy is Acting Laboratory Operations Chief, CECER-LL. The USACERL technical editor was Gloria J. Wienke, Technical Information Team.

COL J ames A. Walter is the Commander of USACERL, and Dr. Michael J . O'Connor is Director. 


\section{Contents}

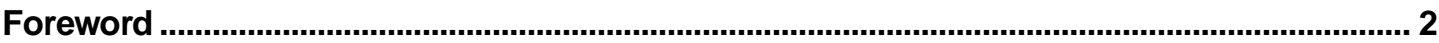

1 Introduction

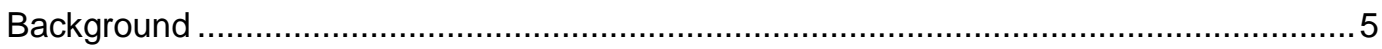

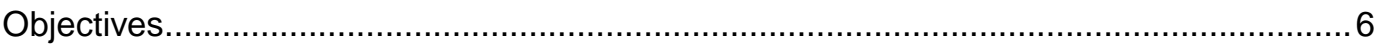

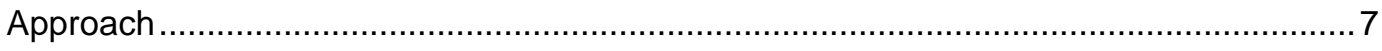

Scope

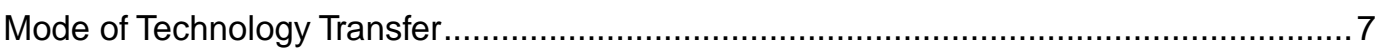

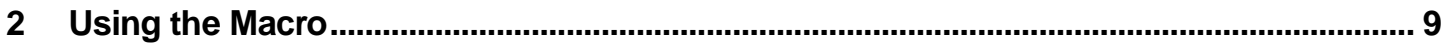

3 Survey Methodology

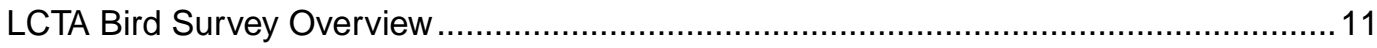

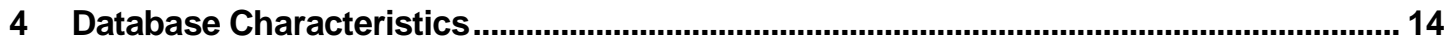

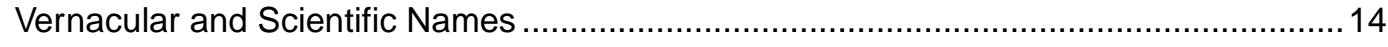

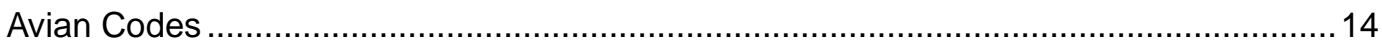

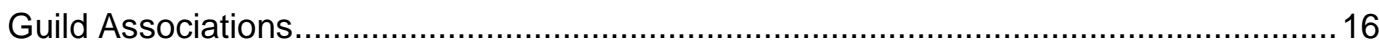

5 Summary

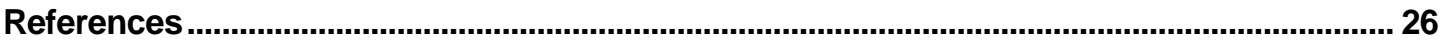

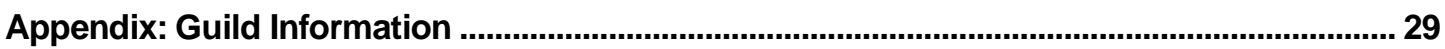

Distribution 


\section{Introduction}

\section{Background}

The U.S. Army is responsible for managing 5 million hectares (12.4 million acres) of land on approximately 120 military installations throughout the United States (Tazik and Pierce 1994). As part of an integrated land management approach and under the sponsorship of the U.S. Army Engineering and Housing Support Center (USAEHSC), the U.S. Army Land Condition Trend Analysis (LCTA) program was developed at the U.S. Army Construction Engineering Research Laboratories (USACERL). The foundation of the LCTA program is the use of standardized methods to collect, analyze, and report natural resources data and is the Army's standard for land inventory and monitoring (Technical Note [TN] 420-74-3 1990). Approximately 50 major military training and testing installations in the United States and Germany have initiated or completed implementation of the LCTA program (Tazik et al. 1992).

In addition to providing sufficient training opportunities, land managers must consider a variety of multiple use demands and endangered species issues on a daily basis. For example, the conservation of biological diversity increasingly is a management goal for many state and federal agencies, including the Army, as outlined in section 11-1(c) of Army Regulation (AR) 200-3 (1995). Unfortunately, the funds to support research efforts under conservation of biological diversity an extremely broad subject area, will likely be difficult to obtain in the future, and installation staff must continue to do more with less. An economic alternative to obtaining new data is to maximize analyses done on existing data from past and current efforts.

Avian guild theory has been reported as a relatively simple option for evaluating impacts of a variety of military land use actions on an installation's avian community (Severinghaus 1981), and can be readily incorporated into a relational database such LCTA. Root (1967) was among the first to discuss the guild concept, defining guilds as "a group of species that exploit the same class of environmental resources in a similar way." A less restrictive definition is provided by Landres (1983), who defines a guild simply as "...a group of species that use a similar resource." An important premise in the guild concept is that wildlife species are associated with structures and/or spatial layers or zones 
within habitats. Moreover, as more layers occur in a community, potentially more niches are included, and more wildlife species likely to occur (Short 1983). Assuming guild members are correctly identified, actions that affect environmental resources will similarly affect the members of the guilds using the resources.

Numerous avian guilds have been recognized or proposed; indude groupings based on nesting substrate/location, feeding strategy, migratory tendency, and general nesting and feeding associations (Short 1983; Terres 1987; Peterjohn and Sauer 1993; Droege and Sauer 1989). Admittedly, the definition and application of guild theory is a point of debate among researchers, but the strong appeal of guild-based summaries is the ability to present plot data from a functional perspective rather than rely solely on species richness (plot, habitat, or installation-wide), similarity indices, and other traditional (numerical) measures of diversity. Moreover, associating species with specific environmental attributes or resources can aid in the risk assessment process. For instance, once the resource(s) to be affected by training activities is known, avian guilds (species) likely to be affected can be readily identified. Avian guilds are easily defined from LCTA survey data and can provide an understanding of how species assemblages change over time relative to a resource. Grouping of species has the added benefit of reducing bias associated with individual species detection rates while effectively increasing sample size and the power (beta) of statistical tests.

\section{Objectives}

The primary objectives of this report are to:

1. Expand the utility of LCTA data by providing published ecological attributes for all bird species that occur in, or those that migrate through, the United States,

2. Describe common guild associations and provide documentation enabling installation natural resources personnel to adjust their LCTA database to reflect bird species characteristic of their installation and region, and

3. Allow the simple conversion of LCTA codes to the more universally recognized Bird Banding Laboratory Alpha codes and American Ornithologists' Union (AOU) numbers, and vice versa. 


\section{Approach}

Information for this database was derived from a variety of sources that included bird field guides (Peterson 1980, 1990), encyclopedias (Terres 1987), and field handbooks (American Ornithologists' Union 1983; Ehrlich, Dobkin, and Wheye 1988). Data quality checks were initiated after species-attribute information had been incorporated to ensure agreement among the various sources. A limited number of new species codes were developed by USACERL to represent species not recognized either by USACERL (Kowalski and Whitworth 1991), the Bird Banding Laboratory (Canadian Fish and Wildlife Service and the U.S. Fish and Wildlife Service 1991), or the American Ornithologists' Union (1983).

\section{Scope}

This report describes an LCTA database update that provides sel ected ecological attributes of 676 bird species occurring within the continental United States. Ecological attributes for each species includes: neotropical status, nest location, nest type, food type, foraging substrate, foraging technique, and general habitat type.

\section{Mode of Technology Transfer}

This report is intended to assist natural resources personnel in customizing and updating their LCTA bird database, thus permitting a greater range of potential analyses and facilitating the interpretation of their LCTA bird data. The database update may also be incorporated into future versions of the automated LCTA frontend program and linked to additional databases. This report represents one in a series of user-friendly reports documenting the utility, limitations, and potential applications of LCTA data (Price at al. 1995; Anderson et al. 1995; Rice, Demarais, and Hansen 1997; Anderson, Guertin, and Price 1996; Whitworth and Hill 1997).

An electronic database containing all guild information and the macro can be obtained from USACERL. For further information about the program, contact the authors at 1-800-USA-CERL, or write to USACERL, Attn: LL-N, P.O. Box 
9005, Champaign, IL 61826-9005. Use of the macro requires Microsoft Excel $97^{*}$ or newer version.

* Citing manufacturer and software names is not intended to imply endorsement by the Department of Defense or the U.S. Army. 


\section{Using the Macro}

After installing the file, open Microsoft ${ }^{\circledR}$ Excel and select $\langle$ bird_db.xls $>$. Refer to the appropriate Microsoft ${ }^{\circledR}$ Office 97 documentation for further details on enabling and using macros. See the Appendix for standard guild information.

Upon initializing the macro, a dialog box prompts you to $\measuredangle$ verwrite existing output data?>. By answering <Yes>, any current data within the Output sheet will be lost. By answering $\langle\mathrm{No}\rangle\rangle$, additions and corrections will simply be appended to existing output data.

The second dialog box prompts you to $\varangle$ nter code type (LCTA, ALPHA, AOU) $>$. These codes are based on either the Land Condition Trend Analysis (LCTA) program, the Bird Banding Laboratory (Alpha), or the American Ornithologist's Union (AOU).

The third dialog box prompts you to $\varangle$ Enter bird species code>. As you enter each code and press $\varangle R E T U R N>$, data is pulled from the bird database and inserted into the Output sheet. Codes that are entered incorrectly, or those that do not correspond to a species within the database, will be flagged as Code Not Found. Do not worry about duplicate or invalid codes at this point; they will be corrected later. Press $\varangle R E T U R N>$ on an empty field when all applicable codes have been entered.

The fourth dialog box prompts you to $\varangle$ nter sorting criteria>. Output data can be sorted according to LCTA code, ALPHA code, AOU number, common name, or scientific name. Duplicate codes are also removed at this point. You can delete rows that show Code Not Found by simply deleting the rows. You can remove these codes at any time.

The fifth dialog box then prompts you to 〈Save the data?>. By answering <Yes>, you can save the output to a specified directory or subdirectory. By answering $\langle\mathrm{No}$, new output data will not be saved. At this point, if you need to correct or verify certain codes, or add species, simply rerun the macro. The macro will then loop back to the initial dialog box and prompt to <Overwrite existing output data?>. If you are simply editing codes or adding new codes, answer $\varangle \mathrm{No}>$. 
Continue rerunning the macro until all species have been included, and all errors identified and corrected.

Now, with the installation-specific guild summaries for each species, verify that all species that occur on the installation have in fact been included in the output file. When all verifications are completed, go back and modify the guild association characteristics for each species. Many species occupy various guilds in different parts of the country, so stated representations for every species may not be appropriate for your installation. Modifying guild characteristics to fit local conditions will make data analysis and interpretation more precise. 


\section{Survey Methodology}

\section{LCTA Bird Survey Overview}

To place guild-based avian summaries in the proper context and define the limitations of the data, it is important to at least present a brief overview of field survey methods. For complete information on LCTA methodologies, refer to the U.S. Army Land Condition Trend Analysis (LCTA) Plot Inventory Field Methods Technical Report (Tazik et al. 1992). Bird surveys are conducted during the morning ( 0 to 4 hrs post sunrise) on a random subsample of all LCTA core plots by using a modified point-count technique. Under typical circumstances, the total number of core plots sampled is 60 . Each plot is surveyed at least once. Initial implementation guidance suggested an evening survey be considered as well (Tazik et al. 1992). Table 1 identifies the three segments constituting a valid plot survey. Briefly, an observer(s) slowly walks the 100-m transect in 6 minutes and records all birds seen and heard within 100-m of the plot (Line Out). Upon reaching the end of the transect the observer stops and for 8 minutes records all birds seen and heard within 100-m (End Point). The observer then walks back to the starting point, again recording all birds seen and heard within 100-m of the plot (Line In). The maximum number of birds exhibiting breeding behavior are totaled by species (MAX Pair, Table 2). Mated status for each individual is described in Table 3. Species observed outside the plot boundaries, migrating overhead, and before/after the official survey times are also recorded on the standard LCTA Bird Survey Data Form (Table 2) within the "Others" column.

For each species, common name, LCTA species code, mated status, and total number of individuals are recorded within each segment of the survey (Table 2). Species documented within the "Others" column should only be used in installation checklists and should not be included in statistical analyses since they did not occur within the designated LCTA plot boundary. The "Others" category represents observations of species outside the plot boundaries, such as migratory birds and raptors, and includes those species observed while driving to and from plots.

Upon completing all bird surveys within a given field season, the data is then entered into a standardized electronic database. The format of the database has 
recently been revised (Table 4) by researchers at USACERL to allow installation personnel the advantage of being able to sort individual occurrences (e.g., by mated status or measurement point).

Table 1. LCTA measure point code with short description.

\begin{tabular}{|cl|}
\hline Measure Point Code & Line Segment \\
LO & Line Out \\
EP & End Point \\
LI & Line In \\
C & All ages and genders of species outside \\
& the plot boundary were totaled and entered \\
& as one observation \\
& \\
\end{tabular}

Table 2. Completed LCTA bird survey data form, USMA, West Point, NY.

Installation_West Point LCTA Plot Number_ 9 Observer_T. Miller Date_5/29/93 Starting time_1902 AM (PM) (circle one)

Cloud Cover $(\%) \quad 10 \quad$ Wind $\quad 10-15 \quad T e m p\left(\mathrm{C}^{\circ}\right) \_16$

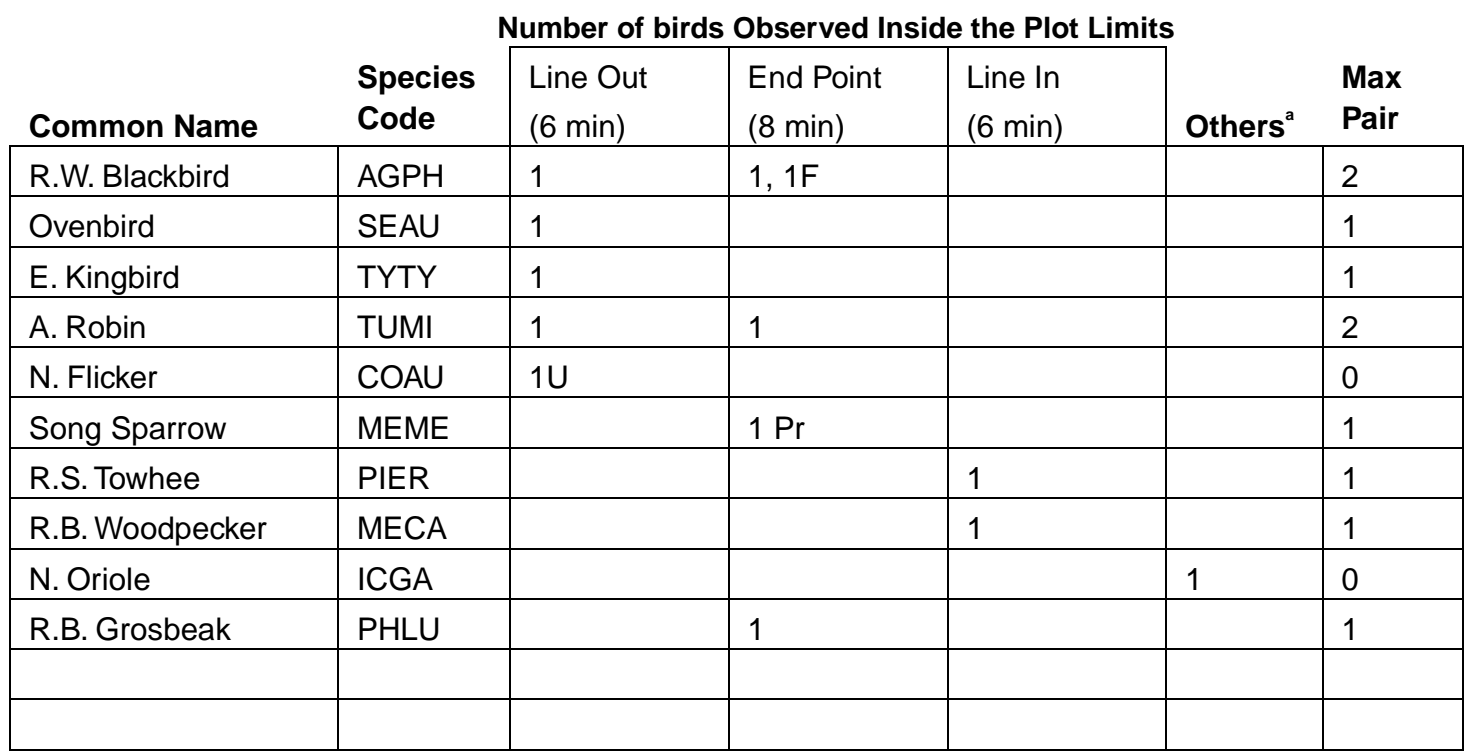

\footnotetext{
${ }^{a}$ This category includes species observed outside the plot limits, and flyovers of birds not using the plot.
} 
Table 3. LCTA mated status code and description.

\begin{tabular}{|cl|}
\hline Mated Status Code & \multicolumn{1}{c|}{ Mated Status } \\
$*$ & Singing Male \\
PR & Adult male/female \\
M & Nonsinging male \\
F & Female only \\
U & Unknown sex and age \\
Y & Young of the year \\
FL & Flock \\
C & All statuses were combined \\
NR & Not recorded \\
& \\
\hline
\end{tabular}

Table 4. Representative spreadsheet within the USACERL LCTA bird database.

\begin{tabular}{|c|c|c|c|c|c|c|c|c|}
\hline Instalid & Plotid & Recdate & Vertid & Period & Num & Flyovers & Mated Status & Measure Point \\
\hline WES & 9 & $5 / 30 / 93$ & AGPH & AM & 4 & $\mathrm{~N}$ & * & LO \\
\hline WES & 9 & $5 / 30 / 93$ & AISP & AM & 1 & $\mathrm{~N}$ & * & EP \\
\hline WES & 9 & $5 / 30 / 93$ & BOLE & AM & 1 & $\mathrm{~N}$ & * & $\mathrm{EP}$ \\
\hline WES & 9 & $5 / 30 / 93$ & BUJA & AM & 1 & $\mathrm{~N}$ & $U$ & LO \\
\hline WES & 9 & $5 / 30 / 93$ & COAU & AM & 1 & $Y$ & * & C \\
\hline WES & 9 & $5 / 30 / 93$ & CYCR & AM & 1 & $\mathrm{~N}$ & * & LO \\
\hline WES & 9 & $5 / 30 / 93$ & HYMU & AM & 1 & $\mathrm{Y}$ & * & C \\
\hline WES & 9 & $5 / 30 / 93$ & ICGA & AM & 1 & $\mathrm{Y}$ & * & $\mathrm{C}$ \\
\hline WES & 9 & $5 / 30 / 93$ & MECA & AM & 1 & $\mathrm{Y}$ & * & C \\
\hline WES & 9 & $5 / 30 / 93$ & MEGE & AM & 1 & $\mathrm{~N}$ & * & $\mathrm{EP}$ \\
\hline WES & 9 & $5 / 30 / 93$ & MEME & AM & 1 & $\mathrm{~N}$ & * & EP \\
\hline WES & 9 & 5/30/93 & MNVA & AM & 1 & $\mathrm{~N}$ & * & LO \\
\hline WES & 9 & $5 / 30 / 93$ & PHLU & AM & 1 & $\mathrm{~N}$ & * & LO \\
\hline WES & 9 & $5 / 30 / 93$ & TYTY & AM & 2 & $\mathrm{~N}$ & PR & EP \\
\hline WES & 9 & $5 / 30 / 93$ & VIFL & AM & 1 & $\mathrm{~N}$ & * & LO \\
\hline WES & 9 & $5 / 30 / 93$ & VIOL & AM & 1 & $\mathrm{~N}$ & * & LO \\
\hline WES & 9 & $5 / 29 / 93$ & AGPH & PM & 1 & $\mathrm{~N}$ & * & LO \\
\hline WES & 9 & $5 / 29 / 93$ & AGPH & PM & 1 & $\mathrm{~N}$ & * & $\mathrm{EP}$ \\
\hline WES & 9 & $5 / 29 / 93$ & AGPH & PM & 1 & $\mathrm{~N}$ & $\mathrm{~F}$ & EP \\
\hline WES & 9 & $5 / 29 / 93$ & COAU & PM & 1 & $\mathrm{~N}$ & $U$ & LO \\
\hline WES & 9 & $5 / 29 / 93$ & ICGA & PM & 1 & $\mathrm{Y}$ & * & C \\
\hline WES & 9 & $5 / 29 / 93$ & MECA & PM & 1 & $\mathrm{~N}$ & * & $\mathrm{LI}$ \\
\hline WES & 9 & $5 / 29 / 93$ & MEME & PM & 2 & $\mathrm{~N}$ & PR & EP \\
\hline WES & 9 & $5 / 29 / 93$ & PHLU & PM & 1 & $\mathrm{~N}$ & * & EP \\
\hline WES & 9 & $5 / 29 / 93$ & PIER & PM & 1 & $\mathrm{~N}$ & * & $\mathrm{LI}$ \\
\hline WES & 9 & $5 / 29 / 93$ & SEAU & PM & 1 & $\mathrm{~N}$ & * & LO \\
\hline WES & 9 & $5 / 29 / 93$ & TUMI & PM & 1 & $\mathrm{~N}$ & * & EP \\
\hline WES & 9 & $5 / 29 / 93$ & TUMI & PM & 1 & $\mathrm{~N}$ & * & LO \\
\hline WES & 9 & $5 / 29 / 93$ & TYTY & PM & 1 & $\mathrm{~N}$ & * & LO \\
\hline
\end{tabular}




\section{Database Characteristics}

\section{Vernacular and Scientific Names}

Vernacular and scientific names were taken from the 1987 Checklist of Vertebrates of the United States, the U.S. Territories, and Canada (hereafter called Checklist of Vertebrates, Banks, McDiarmid, and Gardner 1987). The 1987 checklist includes a national list of standard vertebrate species names that is accurate and up-to-date, as of 1985. However, with the checklist being more than 10 years old, many scientific names may have changed. Also, depending on taxonomic characteristics, certain individuals and organizations lump species while splitting others. For example, the Northern Flicker is often recognized as one unique species by some researchers while others split it into two unique species: the Yellow-shafted Flicker and the Red-shafted Flicker. Refer to Table 5 for additional species that are frequently lumped or split.

Common names are entered in two different formats within the electronic database. The first series of common names is arranged with the last name followed by the first name (e.g., Blackbird, Red-winged). The second series of common names is arranged with the first name followed by the last name (e.g., Red-winged blackbird). Both formats were included within the electronic database so that individuals can opt for either one without having to retype each common name.

\section{Avian Codes}

Three sets of organizational avian codes are included in the database to facilitate communication between various organizations and enable comparisons between data sets. 
Table 5. Species that are typically lumped or split.

\begin{tabular}{|c|c|}
\hline Northern Flicker & $\begin{array}{l}\text { Yellow-shafted Flicker } \\
\text { Red-shafted Flicker }\end{array}$ \\
\hline Snow Goose & $\begin{array}{l}\text { Blue Goose } \\
\text { Snow Goose }\end{array}$ \\
\hline Brant & $\begin{array}{l}\text { Atlantic Brant } \\
\text { Black Brant }\end{array}$ \\
\hline Tricolored Blackbird & $\begin{array}{l}\text { Tawny-shouldered Blackbird } \\
\text { Yellow-shouldered Blackbird }\end{array}$ \\
\hline Scott's Oriole & $\begin{array}{l}\text { Black-vented Oriole } \\
\text { Black-cowled Oriole }\end{array}$ \\
\hline Northern Oriole & $\begin{array}{l}\text { Baltimore Oriole } \\
\text { Bullock's Oriole }\end{array}$ \\
\hline Rosy Finch & $\begin{array}{l}\text { Gray-crowned Rosy Finch } \\
\text { Black Rosy Finch } \\
\text { Black-capped Rosy Finch }\end{array}$ \\
\hline Dark-eyed Junco & $\begin{array}{l}\text { Slate-colored Junco } \\
\text { Oregon Junco } \\
\text { Gray-headed Junco } \\
\text { White-winged Junco }\end{array}$ \\
\hline Yellow-rumped Warbler & $\begin{array}{l}\text { Myrtle Warbler } \\
\text { Audubon's Warbler }\end{array}$ \\
\hline Palm Warbler & $\begin{array}{l}\text { Western Palm Warbler } \\
\text { Yellow Palm Warbler }\end{array}$ \\
\hline Tufted Titmouse & $\begin{array}{l}\text { Eastern Titmouse } \\
\text { Black-crested Titmouse }\end{array}$ \\
\hline Bushtit & $\begin{array}{l}\text { Common Bushtit } \\
\text { Black-eared Bushtit }\end{array}$ \\
\hline
\end{tabular}

\section{LCTA Codes}

LCTA wildlife codes were established in 1991 by USACERL researchers (Kowalski and Whitworth 1991) and based on scientific names from the Checklist of Vertebrates (Banks, McDiarmid, and Gardner 1987). Each code consists of the first two letters of the genus and the first two letters of the specific epithet. To eliminate duplication, numerical values are added to similar four-letter codes (e.g., SICA1 and SICA2). However, a potential problem with using numerical values is that many observers find them difficult to remember and occasionally may enter the wrong one. Unfortunately, a code entered incorrectly may not necessarily show up as an error. Therefore, it is desirabile for a qualified field 
biologist to input and/or check plot data. For example, if an observer records an eastern wood pewee (COVI1) but fails to write the " 1 " on the suffix, the result is not a illegitimate code (with an error message) but represents another species, the northern bobwhite (COVI). In some areas both species can occur together, making this error more difficult to detect. This potential problem can be minimized by the use of experienced field personnel. Alternatively, an installation may want to consider using a more intuitive method such as the more widely accepted AOU Codes (American Ornithologists' Union 1983), converting back to LCTA codes when necessary.

\section{Alpha Codes}

Alpha codes, developed by the Bird Banding Laboratory, consist of a four-letter combination composed of the common name (e.g., BAEA [Bald Eagle] and RWBL [Red-winged Blackbird]: Canadian Fish and Wildlife Service and U.S. Fish and Wildlife Service 1991). Potential problems include: (1) field biologists who devise individual or entire sets of alpha codes instead of following accepted guidelines or established codes, (2) species that do not have an alpha code (e.g., game species like quail, pheasant, turkey, etc.), and (3) common names that vary between different localities. Interestingly, 36 species included in the database did not have a designated alpha code. Therefore, to provide some consistency and completeness, USACERL developed codes for the 36 undesignated species, marking each with an asterisk (*).

\section{AOU Codes}

AOU codes, established by the American Ornithologists' Union in the late 1800s, are numerical codes consisting of four numbers (American Ornithologists' Union 1983). Since the AOU checklist is widely used as an official, or semi-official reference, little change in taxonomic concepts has been implemented, unless widely accepted based on published evidence.

\section{Guild Associations}

Information within this section was derived primarily from the 1988 Birder's Handbook (Ehrlich, Dobkin, and Wheye 1988).

Many species can substitute or switch nest location, nest type, diet, foraging substrate, foraging technique, and habitat type; therefore, ecological guild-based attributes for some species may not be appropriate in every locality. Some species are "specialists" and associate with a single guild, whereas other species 
are "generalists" and associate with multiple guilds. For example, a species may frequent multiple habitats, consume a variety of foods, construct a nest in a variety of locations, but builds only one type of nest. Depending on the species and geographic area, some guild data will likely have to be modified to fit local conditions. The appendix contains standard guild information.

\section{Neotropical Migrant Status}

The neotropical bird classification system listed bel ow (Partners in Flight 1991) has been included in the database to help installation personnel become more familiar with migratory species. The 676 bird species that are currently listed in the database are divided as follows:

Class A - 141 species; Species that breed in North America and spend their nonbreeding period primarily south of the United States.

Class B - 71 species; Species that breed and winter extensively in North America, but some populations winter south of the United States.

Class C - 34 species; Species whose breeding range is primarily south of the U.S./Mexico border and enters the United States al ong the Rio Grande Valley.

Class D - 5 species; Species whose breeding range is restricted to the Florida Peninsula within the United States, and withdraws from Florida during the nonbreeding season.

Class R - 425 species; Species that breed and winter exclusively in the United States (resident species).

Class A and B neotropical migrant species are probably most vulnerable to habitat alterations since they spend their nonbreeding period south of the United States, and migrate across several environments to reach their breeding grounds. Class C and Class D neotropical migrant species are also important but typically breed south of the United States. Class R (resident species) do not migrate but are still being threatened by alteration and degradation of their habitats beyond the point of use by a particular species within the United States.

\section{Nest Location}

Nest locations have been generalized for most species; however, some specialized nest locations have been included for those species that will nest only in certain areas. Thirteen nest locations are described below: 
Bank - Includes river banks, areas of soft soil on steep island slopes, etc., where nest burrows are excavated.

Cactus - Includes nests built in cholla and yucca trees.

Cave - Nest built within a cave.

Cliff - Includes nests situated in natural crevices or on ledges of cliffs typically offering a commanding view of a defensible position, and sometimes chosen when no suitable trees are available.

Floating - Includes nests floating on water and usually anchored to live emergent or submerged vegetation.

Ground - Includes nests placed among the roots, or in niches among the roots of fallen trees, among tules and reeds (in marshes), among grasses, on bare rock, or simply scraped in the dirt or sand.

MMS (Man-made Structure) - Includes nests placed among any man-made structure (bridges, barn, etc.).

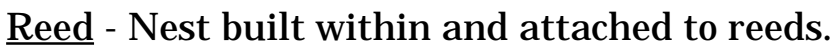

Shrub - Includes nests placed within any multi-stemmed woody plant that does not have a distinct single trunk extending several feet between the ground and the lowest branching point.

Snag - Includes nests placed in a standing dead tree. Also used for species that use cavities in dead and live trees more/less indiscriminately.

Vine Tangle - Includes nests placed in vines, brambles, brush piles, etc.

Woody Lower Canopy - Includes nests placed in the lower canopy of deciduous or coniferous trees.

Woody Upper Canopy - Includes nests placed in the upper canopy of deciduous or coniferous trees. 


\section{Nest Type}

Birds of the same species typically construct similar nests; however, the materials available often differ from area to area. Thirteen nest types are characterized below:

Abandoned - Nest is built in an abandoned nest previously built by another species.

Burrow - Eggs placed in a chamber at the end of a tunnel. Tunnels are either excavated by the bird (most kingfishers, puffins, storm petrels) or usurped from small mammals, especially ground squirrels and prairie dogs.

Cavity - Eggs placed in a cavity that has been either excavated (woodpeckers) or in a natural cavity found in a dead or dying tree. Sometimes a cup or other structure is built within.

Crevice - Eggs placed in a crack in the face of a cliff, between boulders, in a manmade structure, etc.

Cup - A sometimes bulky, but always deep depression, with a hemispherical inside and a rim height several times the diameter of the eggs.

None - No nest is constructed. Eggs are typically layed on ground with nothing to protect them.

Oven - Oven-shaped (roofed or arched) nest on the ground.

Parasitic - No nest is constructed. Eggs are deposited in the nest of another species.

Pendant - An elongate saclike nest suspended from a branch.

Platform - A structure in a tree, on a cliff, or providing a dry place above marshy ground or water, usually big enough for the bird to land on, with or without a distinct depression to hold the eggs. Typical of many raptors and wetland birds.

Saucer - A shallow cup with the height of the rim not more than twice the diameter of the eggs. Also a flattened nest of pliable vegetation as in some wetland birds. 
Scrape - A simple depression usually with a rim sufficient to prevent eggs from rolling away. Those of many duck species are almost bowl-shaped. Occasionally with lining added.

Sphere - Globe-shaped or ball-shaped. A roughly round structure, fully enclosed except a small opening usually on the side or at one end.

\section{Food Type}

Diet is a category that is often difficult to quantify. Species rely on a variety of food types throughout the year and many species have an entirely different winter diet than that of the summer and spring. Therefore, inferences that follow need to be looked at as generalizations and will not represent the diet of a particular species in every geographic area within all seasons. Thirteen food items are listed below:

Aquatic Inverts - May include aquatic insects, crayfish, shrimp, snails, bivalves, etc.

Birds - Includes birds and their eggs.

Carrion - Dead and putrefying flesh.

Fish - Includes fish, their fry, and eggs.

Fruit - Includes fruit and berries.

Greens - May include leafy parts of both aquatic and terrestrial plants, and bulbs.

Insects - May include insects, spiders, mites, land snails, slugs, worms, millipedes, sowbugs, etc.

Nectar - The sugar-containing liquid secretion of the nectary of many flowers.

Nuts - A type of fruit with one seed and a hard woody outer layer (e.g., acorns and beechnuts).

Seeds - Includes grains, sunflower seeds, conifer seeds, etc.

Small Mammals - Any animal of the class Mammalia. Includes shrews, ground squirrels, rabbits, but most often rodents. 
Small Verts - Includes reptiles (e.g., lizards, snakes) and amphibians (e.g., salamanders, frogs).

Tree Sap - A watery solution that circulates through a plant's vascular system.

\section{Foraging Substrate}

Foraging substrate has been included to aide in making larger scale generalizations with regard to feeding techniques. Five substrates have been identified:

Air - Foraging done in the air. (Includes foraging techniques such as: Aerial Foraging, Aerial Pursuit, Hawker, Hover \& Pick, Hover Gleaner, and Piracy.).

Bark - F oraging done primarily on the bark of trees. (Includes: Bark Gleaner.)

Foliage - Foraging done primarily on foliage. (Includes: Foliage Browser and Foliage Gleaner.)

Ground - Foraging done primarily on the ground. (Includes: Digging, Ground Gleaner, High Patrol, Hover \& Pounce, Low Patrol, Piracy, Scavenger and Swooper.)

Water - Foraging done primarily on or in the water. (Includes: Ambusher, Dabbler, High Dives, Prober, Skimmer, Surface Dips, and Surface Dives.)

\section{Foraging Technique}

Many birds that eat both seeds and insects will take proportionally more insects when available. Therefore, geographic area and availability of food items will have a major effect on items consumed. In addition, both primary and secondary foraging techniques may be used to obtain primary food items. Twenty-two foraging techniques have been identified:

Aerial Foraging - Capturing flying insects while in a prolonged, continuous flight.

Aerial Pursuit - Chasing or catching birds in midair, stooping (dropping on flying birds from above, killing them in midair with a blow from the talons), or snatching them from their perches. 
Ambusher - Hunting by standing motionless on a bank or in the water and spearing fish, frogs, etc.

Bark Gleaner - Gleaning from tree trunks and branches. Includes excavating and drilling into bark.

Dabbler - Floating on the surface in shallow water, pivoting headfirst downward while raising hind-quarters above the water to reach submerged plants or animals on or near the substrate.

Digging - Includes scratching at the surface and digging for bulbs, insects, etc.

Foliage Browser - Browsing tender shoots, twigs, and leaves of trees and shrubs for food.

Foliage Gleaner - Gleaning from foliage and occasionally from branches. Take invertebrates and/or fruit from the vegetation, not from the ground surface.

Ground Gleaner - Picking up items from the surface of soil, turf, sand, etc.

Hawker - Sallies from perch on short flights to capture flying insects.

High Dives - Dropping from a height into the water, usually to catch fish, but sometimes to take waterfowl and other prey.

High Patrol - Soaring at high altitudes in search of carrion or prey.

Hover \& Pick - Hovering above a particular substrate, then snatching or picking up the food item.

Hover \& Pounce - Hovering before swooping or dropping down on prey.

Hover Gleaner - Taking nectar, insects, or berries from plants above the ground while hovering.

Low Patrol - Seeking prey in a low searching flight.

Piracy - Taking prey from another bird.

Scavenger - Feeding on carrion, refuse, or material left unconsumed by other organisms. 
Skimmer - Flying low over the water and snatching up fish or aquatic invertebrates.

Surface Dips - Taking food from the water's surface or from just below while floating or swimming on the surface.

Surface Dives - Floating and then diving under water using feet and/or wings in search of prey.

Swooper - Snatching prey from the ground in talons after a gliding descent from perch with wings spread.

\section{General Habitat}

General habitat is another category that is often quite difficult to define. Many species frequent several habitat types and are not associated with just one. Therefore, general habitat classifications within the database should be viewed with caution. Fifteen habitat types are listed below:

Coastal - An area of land near the shore (Pacific and Atlantic Coast).

Cropland - Includes lands that have been planted with grain or sorghum, disked fields, or anything el se pertaining to agricultural practices.

Desert - A terrestrial environment characterized by poor soil, climatic extremes of high and low temperatures, and low rainfall.

Forest - An extensive concentration of trees and related vegetation. Includes sparsely wooded areas, densely wooded areas, and pinon-juniper associations.

Forest Edge - The transitional area where a forest ends and another distinct habitat begins.

Freshwater - Includes areas such as lakes, ponds, rivers, and streams.

Grassland - An area of land on which the natural dominant plant forms are grasses and forbs. Areas with scattered trees and/or snags are included in this community type.

Pelagic - Living or occurring in the open sea for a substantial portion of their lives. 
Riparian - An area located along the bank of a natural watercourse (as a river) or sometimes of a lake or tidewater.

Rocky Slope - An area abounding in or consisting primarily of rocks (mountains, cliffs, talus slopes, etc.).

Shoreline - The strip of land where a body of water and the shore meet.

Shrubland - An area of land on which the natural dominant plant forms are shrubs (a low, usually several-stemmed woody plant).

Swamp/Marsh - A wet, spongy land, saturated and sometimes partially or intermittently covered with water. Also includes tracts of soft, wet land, typically characterized with grasses and cattails.

Tundra - A treeless plain that is characteristic of arctic and subarctic regions, and consists of black mucky soil with a permanently frozen subsoil, and supports a dense growth of often conspicuously flowering dwarf herbs.

Urban - Any area habituated with people. Includes cities, towns, suburbs, and possibly farm houses, etc. 


\section{Summary}

Information within this report will provide land managers the capability to: (1) generate ecological guild-based summaries with no additional fieldwork, (2) identify specific groups of birds most likely to be impacted by a spacially descrete training impact, (3) customize their database by adjusting individual attributes for species specific to their installation, and (4) conveniently cross reference, or simply convert, all LCTA species codes to more globally recognized American Ornithological Union "AOU numbers" or the Bird Banding Laboratory "Alpha codes".

The LCTA bird database provides selected ecological attributes of 676 species occurring within the continental United States. However, LCTA avian codes were based on the 1987 Checklist of Vertebrates, which encompasses 1,098 bird species, and covers a wider geographical area. Therefore, depending on installation locality, species range, and occurrence of infrequently observed species, additional species may need to be incorporated into your local database.

Each species within the database has information pertaining to neotropical status, nest location, nest type, diet, foraging substrate, foraging technique, and general habitat. However, since many species occupy various guilds in different parts of the country, stated representations for each species may not accurately portray the most applicable data. Therefore, after all codes have been entered into the avian macro, verified, and an installation-specific database developed, personnel at each installation should go through the guild data for their particular species and make appropriate modifications. 


\section{References}

American Ornithologists' Union. 1983. Check-list of North American Birds, 6th edition. Allen Press, Inc.

Anderson, A.B., P.J Guertin, and D.L. Price, Land Condition Trend Analysis Data: Power Analysis, Technical Report 97/05/ADA 321295 (U.S. Army Construction Engineering Research Laboratories [USACERL], October 1996).

Anderson, A.B., W. Sprouse, D. Kowalski, and R. Brozka, Land Condition Trend Analysis Data Collection Software Users Manual: Version 1.0, ADP Report 95/13/ADA 299981 (USACERL, J uly 1995).

Army Regulation [AR] 200-3, Natural Resources--Land, Forest and Wildlife Management (Headquarters, Department of the Army [HQDA], Washington, DC, February 1995).

Banks, R.C., R.W. McDiarmid, and A.L. Gardner. 1987. Checkl ist of Vertebrates of the United States, the U.S. Territories, and Canada. U.S. Fish and Wildlife Service, Resource Publication 166.

Canadian Fish and Wildlife Service and U.S. Fish and Wildlife Service. 1991. North American Bird Banding. U.S. Department of Interior, Fish and Wildlife Service, Laurel, MD; and Ottawa, Canada: Environment Canada, Canadian Wildlife Service.

Droege, S., and J .R. Sauer, North American Breeding Bird Survey Annual Summary 1988. U.S. Fish and Wildlife Service, Biological Report 89(13), (1989) 16pp.

Ehrlich, P.R., D.S. Dobkin, and D. Wheye. 1988. TheBirder's Handbook: A Field Guide to the Natural History of North American Birds. Simon and Schuster, Fireside, 785pp.

Kowalski, D.G. and W.R. Whitworth. 1991. LCTA species code database devel oped by the U. S. Army Construction Engineering Research Laboratories.

Landres, P.B. "Use of the Guild Concept in Environmental I mpact Assessment," Environmental Management, Vol. 7 (1983), pp 393-398.

Partners in Flight. 1991. Annual Report, Preliminary Lists of Migrants for Neotropical Migrant Bird Conservation Program.

Peterjohn, B.G., and J .R. Sauer, "North American Breeding Bird Survey Annual Summary 19901991," Bird Populations, Vol. 1 (1993), pp 1-16.

Peterson, R.T. 1990. Peterson Fiedd Guides: Western Birds. Houghton Mifflin Company, Boston, 432pp. 
Peterson, R.T. 1980. Peterson Field Guides: Eastern Birds. Houghton Mifflin Company, Boston, 384pp.

Price, D.L., A.A. Anderson, W.R. Whitworth, and P.J . Guertin. Land Condition Trend Analysis Data Summaries. Technical Report-95/39/ADA 300753 (USACERL, September 1995).

Rice, C.G., S. Demarais, and R.W. Hansen, Analysis of LCTA Methods for I nventory and Monitoring Birds and Small Mammals on Army Lands in theSouthwestern United States, Technical Report-97/97/ADA 327767 (USACERL, J une 1997).

Root, R.B. "The niche exploration pattern of the blue-gray gnatcatcher, Ecological Monographs, Vol 37 (1967), pp 317-350.

Severinghaus, W.D. 1981. "Guild Theory Development as a Mechanism for Assessing Environmental Impact." Environmental Management, vol 5:187-190.

Short, H.L. 1983. Wildlife Guilds in Arizona Habitats. USDI, Bureau of Land Management Technical Note 362 (F ort Collins, CO).

Tazik, D.J . and P. Pierce, "U.S. Army, Proceedings--I nteragency E ndangered Species Symposi um," D.J . Tazik, C. Martin, P. Pierce, and J . Ruth, eds., Washington, DC, 26 and 28 August 1994, pp 34-37.

Tazik, D.J ., S.D. Warren, V.E. Diersing, R.B. Shaw, R.J . Brozka, C.A. Bagley, and W.R. Whitworth, "U.S. Army Land Condition-Trend Analysis (LCTA) Plot I nventory Field Methods," Technical Report N-92/03/ADA 277931 (USACERL, February 1992).

Technical Note [TN ] 420-74-3. Army Land Inventory and Monitoring Procedures on Military Installations. Department of theArmy, U.S. Army Engineering and Housing Support Center, Fort Belvoir, VA. 26 November 1990.

Terres, J .K. 1987. TheAudubon Society Encyclopedia of North American Birds. Alfred A. Knopf, Inc., New York. 1109pp.

Whitworth, W.R., and A. Hill, Applicability of Land Condition Trend Analysis Data for Biological Diversity Assessment in the Southeastern United States, Technical Report 97/67/ADA 327477 (USACERL April 1997). 


\section{Appendix: Guild Information}




\begin{tabular}{|c|c|c|c|c|c|c|}
\hline COMMON NAME & SCIENTIFIC NAME & $\begin{array}{l}\text { LCTA } \\
\text { CODE }\end{array}$ & $\begin{array}{l}\text { ALPHA } \\
\text { CODE }\end{array}$ & AOU\# & $\begin{array}{l}\text { NEO } \\
\text { STAT }\end{array}$ & NEST LOCATION \\
\hline Albatross, Black-footed & Diomedea nigripes & DINI & BFAL & 0810 & $\mathrm{R}$ & Ground \\
\hline Albatross, Laysan & Diomedea immutabilis & DIIM & LAAL & 0821 & $\mathrm{R}$ & Ground \\
\hline Anhinga & Anhinga anhinga & ANAN & $\mathrm{ANHI}$ & 1180 & $\mathrm{R}$ & Shrub, Woody Lower Canopy \\
\hline Ani, Groove-billed & Crotophaga sulcirostris & CRSU & GBAN & 3840 & $\mathrm{C}$ & Woody Lower Canopy \\
\hline Ani, Smooth-billed & Crotophaga ani & CRAN & SBAN & 3830 & $\mathrm{R}$ & Woody Lower Canopy \\
\hline Auklet, Cassin's & Ptychoramphus aleuticus & PTAL & CAAU & 0160 & $\mathrm{R}$ & Ground, Cliff \\
\hline Auklet, Crested & Aethia cristatella & AECR & CRAU & 0180 & $\mathrm{R}$ & Ground, Cliff \\
\hline Auklet, Least & Aethia pusilla & AEPU & LEAU & 0200 & $\mathrm{R}$ & Ground, Cliff \\
\hline Auklet, Parakeet & Cyclorrhynchus psittacula & CYPS & PAAU & 0170 & $\mathrm{R}$ & Ground, Cliff \\
\hline Auklet, Rhinoceros & Cerorhinca monocerata & CEMO & RHAU & 0150 & $\mathrm{R}$ & Ground \\
\hline Auklet, Whiskered & Aethia pygmaea & AEPY & WHAU & 0190 & $\mathrm{R}$ & Ground \\
\hline Avocet, American & Recurvirostra americana & REAM & AMAV & 2250 & $\mathrm{R}$ & Ground \\
\hline Becard, Rose-throated & Pachyramphus aglaiae & PAAG & RTBE & 4411 & $\mathrm{C}$ & Woody Upper Canopy \\
\hline Bittern, American & Botaurus lentiginosus & BOLE & AMBI & 1900 & $\mathrm{R}$ & Ground \\
\hline Bittern, Least & Ixobrychus exilis & IXEX & LEBI & 1910 & $\mathrm{R}$ & Ground, Shrub \\
\hline Blackbird, Brewer's & Euphagus cyanocephalus & EUCY & BRBL & 5100 & $\mathrm{~B}$ & Woody Upper Canopy, Ground \\
\hline Blackbird, Red-winged & Agelaius phoeniceus & AGPH & RWBL & 4980 & $\mathrm{~B}$ & Reed, Shrub \\
\hline Blackbird, Rusty & Euphagus carolinus & EUCA1 & RUBL & 5090 & $\mathrm{R}$ & Woody Lower Canopy, Shrub \\
\hline Blackbird, Tricolored & Agelaius tricolor & AGTR & TRBL & 5000 & $\mathrm{R}$ & Reed, Shrub \\
\hline Blackbird, Yellow-headed & Xanthocephalus xanthocephalus & XAXA & YHBL & 4970 & A & Reed, Shrub \\
\hline Bluebird, Eastern & Sialia sialis & SISI & $\mathrm{EABL}$ & 7660 & B & Snag, Woody Upper Canopy \\
\hline Bluebird, Mountain & Sialia currucoides & SICU & MOBL & 7680 & B & Snag, Woody Upper Canopy \\
\hline Bluebird, Western & Sialia mexicana & SIME & WEBL & 7670 & B & Snag, Woody Upper Canopy \\
\hline Bluethroat & Luscinia svecica & LUSV & BLUE & 7640 & $\mathrm{R}$ & Ground \\
\hline Bobolink & Dolichonyx oryzivorus & DOOR & ВOBO & 4940 & A & Ground \\
\hline Bobwhite, Northern & Colinus virginianus & COVI & NOBO* & 2890 & $\mathrm{R}$ & Ground \\
\hline Booby, Blue-footed & Sula nebouxii & SUNE & BFBO & 1141 & $\mathrm{R}$ & Ground \\
\hline Booby, Brown & Sula leucogaster & SULE & BRBO & 1150 & $\mathrm{R}$ & Ground \\
\hline Booby, Masked & Sula dactylatra & SUDA & MABO & 1140 & $\mathrm{R}$ & Ground \\
\hline Booby, Red-footed & Sula sula & SUSU & RFBO & 1160 & $\mathrm{R}$ & Woody Upper Canopy, Ground \\
\hline Brant & Branta bernicla & BRBE & BRAN $^{*}$ & 1730 & $\mathrm{R}$ & Ground \\
\hline Bufflehead & Bucephala albeola & BUAL & BUFF & 1530 & $\mathrm{R}$ & Snag, Bank \\
\hline Bunting, Indigo & Passerina cyanea & PACY & INBU & 5980 & A & Shrub, Woody Lower Canopy \\
\hline Bunting, Lark & Calamospiza melanocorys & CAME & LARB & 6050 & A & Ground \\
\hline Bunting, Lazuli & Passerina amoena & PAAM1 & LASB & 5990 & A & Shrub, Woody Lower Canopy \\
\hline Bunting, McKay's & Plectrophenax hyperboreus & PLHY & MKBU & 5350 & $\mathrm{R}$ & Ground, Cliff \\
\hline Bunting, Painted & Passerina ciris & PACl1 & PABU & 6010 & A & Shrub, Woody Lower Canopy \\
\hline Bunting, Snow & Plectrophenax nivalis & PLNI & SNBU & 5340 & $\mathrm{R}$ & Ground, Cliff \\
\hline Bunting, Varied & Passerina versicolor & PAVE & VABU & 6000 & $\mathrm{C}$ & Shrub, Woody Lower Canopy \\
\hline Bushtit & Psaltriparus minimus & PSMI & $\mathrm{BUTI}^{*}$ & 7430 & $\mathrm{R}$ & Woody Lower Canopy, Shrub \\
\hline Canvasback & Aythya valisineria & AYVA & CANV & 1470 & $\mathrm{R}$ & Reed, Floating \\
\hline Caracara, Crested & Polyborus plancus & POPL & CRCA & 3620 & $\mathrm{R}$ & Woody Upper Canopy, Shrub \\
\hline Cardinal, Northern & Cardinalis cardinalis & CACA4 & NOCA & 5930 & $\mathrm{R}$ & Shrub, Woody Lower Canopy \\
\hline Catbird, Gray & Dumetella carolinensis & DUCA & GRCA & 7040 & $A$ & Shrub, Woody Lower Canopy \\
\hline Chachalaca, Plain & Ortalis vetula & ORVE & $\mathrm{PLCH}^{*}$ & 3110 & $\mathrm{R}$ & Woody Lower Canopy \\
\hline Chat, Yellow-breasted & Icteria virens & ICVI & $\mathrm{YBCH}$ & 6830 & $A$ & Shrub, Woody Lower Canopy \\
\hline Chickadee, Black-capped & Parus atricapillus & PAAT & $\mathrm{BCCH}$ & 7350 & $\mathrm{R}$ & Woody Lower Canopy, Snag \\
\hline Chickadee, Boreal & Parus hudsonicus & PAHU & $\mathrm{BOCH}$ & 7400 & $\mathrm{R}$ & Woody Lower Canopy, Snag \\
\hline
\end{tabular}




\begin{tabular}{|c|c|c|c|c|c|}
\hline $\begin{array}{l}\text { LCTA } \\
\text { CODE }\end{array}$ & NEST TYPE & FOOD TYPE & $\begin{array}{l}\text { FORAGING } \\
\text { SUBSTRATE }\end{array}$ & FORAGING TECHNIQUE & GENERAL HABITAT \\
\hline DINI & Scrape & Fish, Aquatic Inverts & Water & Scavenger, Ground Gleaner & Pelagic \\
\hline DIIM & Scrape & Aquatic Inverts & Water & Ground Gleaner & Pelagic \\
\hline ANAN & Platform & Fish, Aquatic Inverts & Water & Surface Dives & Freshwater, Swamp/Marsh \\
\hline CRSU & Cup & Insects, Small Verts & Ground & Ground Gleaner & Shrubland, Swamp/Marsh \\
\hline CRAN & Cup & Insects, Small Verts & Ground & Ground Gleaner & Shrubland, Grassland \\
\hline PTAL & Burrow, Crevice & Aquatic Inverts & Water & Surface Dives & Coastal, Pelagic \\
\hline AECR & Crevice & Aquatic Inverts & Water & Surface Dives & Coastal, Pelagic \\
\hline AEPU & Crevice & Aquatic Inverts & Water & Surface Dives & Coastal, Pelagic \\
\hline CYPS & Crevice & Aquatic Inverts, Fish & Water & Surface Dives & Coastal, Pelagic \\
\hline CEMO & Burrow & Fish, Aquatic Inverts & Water & Surface Dives & Coastal, Pelagic \\
\hline AEPY & Crevice & Aquatic Inverts & Water & Surface Dives & Coastal, Pelagic \\
\hline REAM & Scrape & Aquatic Inverts, Insects & Water & Prober & Swamp/Marsh, Freshwater \\
\hline PAAG & Sphere & Insects, Fruit & Air & Hover Gleaner, Hawker & Forest, Shrubland \\
\hline BOLE & Platform & Fish, Aquatic Inverts & Water, Ground & Ambusher, Ground Gleaner & Swamp/Marsh \\
\hline IXEX & Platform & Fish, Aquatic Inverts & Water, Ground & Ambusher, Ground Gleaner & Swamp/Marsh \\
\hline EUCY & Cup & Insects, Seeds & Ground, Foliage & Ground Gleaner, Foliage Gleaner & Shrubland, Riparian \\
\hline AGPH & Cup & Insects, Seeds & Ground, Foliage & Ground Gleaner, Foliage Gleaner & Swamp/Marsh, Riparian \\
\hline EUCA1 & Cup & Insects, Seeds & Ground & Ground Gleaner & Swamp/Marsh, Riparian \\
\hline AGTR & Cup & Insects, Seeds & Ground, Foliage & Ground Gleaner, Foliage Gleaner & Swamp/Marsh, Cropland \\
\hline XAXA & Cup & Insects, Seeds & Ground, Air & Ground Gleaner, Hawker & Swamp/Marsh \\
\hline SISI & Cavity & Insects, Fruit & Air, Foliage & Hawker, Foliage Gleaner & Forest Edge, Grassland \\
\hline SICU & Cavity & Insects, Fruit & Ground & Swooper, Hover \& Pounce & Forest, Grassland \\
\hline SIME & Cavity & Insects, Fruit & Air, Foliage & Hawker, Foliage Gleaner & Riparian, Grassland \\
\hline LUSV & Cup & Insects, Seeds & Ground & Ground Gleaner & Tundra, Rocky Slope \\
\hline DOOR & Cup & Insects, Seeds & Ground, Foliage & Ground Gleaner, Foliage Gleaner & Grassland, Swamp/Marsh \\
\hline COVI & Scrape & Greens, Seeds & Ground & Ground Gleaner & Forest Edge, Shrubland \\
\hline SUNE & Scrape & Fish & Water & High Dives & Coastal, Pelagic \\
\hline SULE & Scrape & Fish & Water & High Dives & Coastal, Pelagic \\
\hline SUDA & Scrape & Fish & Water & High Dives & Coastal, Pelagic \\
\hline SUSU & Platform, Scrape & Fish & Water & High Dives, Skimmer & Coastal, Pelagic \\
\hline BRBE & Scrape & Greens, Aquatic Inverts & Water & Surface Dips, Dabbler & Tundra, Shoreline \\
\hline BUAL & Cavity, Burrow & Aquatic Inverts & Water & Surface Dives & Freshwater, Forest \\
\hline PACY & Cup & Insects, Seeds & Foliage, Ground & Foliage Gleaner, Ground Gleaner & Forest Edge, Shrubland \\
\hline CAME & Cup & Insects, Seeds & Ground, Air & Ground Gleaner, Hawker & Grassland, Shrubland \\
\hline PAAM1 & Cup & Insects, Seeds & Ground, Foliage & Ground Gleaner, Foliage Gleaner & Shrubland, Riparian \\
\hline PLHY & Crevice, Cavity & Insects, Seeds & Ground & Ground Gleaner & Shoreline, Tundra \\
\hline PACl1 & Cup & Seeds, Insects & Ground, Foliage & Ground Gleaner, Foliage Gleaner & Shrubland, Riparian \\
\hline PLNI & Crevice, Cavity & Insects, Seeds & Ground & Ground Gleaner & Shoreline, Tundra \\
\hline PAVE & Cup & Insects, Seeds & Ground, Foliage & Ground Gleaner, Foliage Gleaner & Shrubland \\
\hline PSMI & Pendant & Insects, Seeds & Foliage, Bark & Foliage Gleaner, Bark Gleaner & Forest, Shrubland \\
\hline AYVA & Cup & Greens, Seeds & Water & Surface Dives, Prober & Swamp/Marsh, Freshwater \\
\hline POPL & Platform & Carrion, Small Verts & Ground & Scavenger, Ground Gleaner & Shrubland \\
\hline CACA4 & Cup & Insects, Seeds & Ground & Ground Gleaner & Shrubland, Urban \\
\hline DUCA & Cup & Insects, Fruit & Ground, Foliage & Ground Gleaner, Foliage Gleaner & Shrubland, Riparian \\
\hline ORVE & Saucer & Fruit, Insects & Foliage, Ground & Foliage Gleaner, Ground Gleaner & Riparian, Forest Edge \\
\hline ICVI & Cup & Insects, Fruit & Foliage & Foliage Gleaner & Shrubland, Riparian \\
\hline PAAT & Cavity & Insects, Seeds & Foliage, Bark & Foliage Gleaner, Bark Gleaner & Forest, Riparian \\
\hline PAHU & Cavity & Insects, Seeds & Foliage, Bark & Foliage Gleaner, Bark Gleaner & Forest \\
\hline
\end{tabular}




\begin{tabular}{|c|c|c|c|c|c|c|}
\hline COMMON NAME & SCIENTIFIC NAME & $\begin{array}{l}\text { LCTA } \\
\text { CODE }\end{array}$ & $\begin{array}{l}\text { ALPHA } \\
\text { CODE }\end{array}$ & AOU\# & $\begin{array}{l}\text { NEO } \\
\text { STAT }\end{array}$ & NEST LOCATION \\
\hline Chickadee, Carolina & Parus carolinensis & PACA2 & $\mathrm{CACH}$ & 7360 & $\mathrm{R}$ & Woody Lower Canopy, Snag \\
\hline Chickadee, Chestnut-backed & Parus rufescens & PARU & $\mathrm{CBCH}$ & 7410 & $\mathrm{R}$ & Snag, Woody Lower Canopy \\
\hline Chickadee, Mexican & Parus sclateri & PASC & $\mathrm{MECH}$ & 7370 & $\mathrm{R}$ & Snag, Woody Upper Canopy \\
\hline Chickadee, Mountain & Parus gambeli & PAGA & $\mathrm{MOCH}$ & 7380 & $\mathrm{R}$ & Woody Lower Canopy, Snag \\
\hline Chuck-will's-widow & Caprimulgus carolinensis & CACA3 & CWWI & 4160 & A & Ground \\
\hline Chukar & Alectoris chukar & $\mathrm{ALCH}$ & $\mathrm{CHUK}^{*}$ & 2882 & $\mathrm{R}$ & Ground \\
\hline Condor, California & Gymnogyps californianus & GYCA1 & CALC & 3240 & $\mathrm{R}$ & Cliff \\
\hline Coot, American & Fulica americana & FUAM & AMCO & 2210 & $\mathrm{R}$ & Floating \\
\hline Cormorant, Brandt's & Phalacrocorax penicillatus & PHPE1 & BRAC & 1220 & $\mathrm{R}$ & Cliff, Ground \\
\hline Cormorant, Double-crested & Phalacrocorax auritus & PHAU & DCCO & 1200 & $\mathrm{R}$ & Ground, Woody Upper Canopy \\
\hline Cormorant, Great & Phalacrocorax carbo & PHCA & GRCO & 1190 & $\mathrm{R}$ & Cliff, Woody Upper Canopy \\
\hline Cormorant, Olivaceous & Phalacrocorax olivaceus & PHOL & OLCO & 1210 & $\mathrm{R}$ & Shrub, Woody Lower Canopy \\
\hline Cormorant, Pelagic & Phalacrocorax pelagicus & PHPE & PECO & 1230 & $\mathrm{R}$ & Cliff, Ground \\
\hline Cormorant, Red-faced & Phalacrocorax urile & PHUR & RFCO & 1240 & $\mathrm{R}$ & Cliff \\
\hline Cowbird, Bronzed & Molothrus aeneus & MOAE & BROC & 4960 & C & Woody Upper Canopy, Shrub \\
\hline Cowbird, Brown-headed & Molothrus ater & MOAT & $\mathrm{BHCO}$ & 4950 & B & Woody Upper Canopy, Shrub \\
\hline Crane, Sandhill & Grus canadensis & GRCA1 & SACR & 2060 & $\mathrm{R}$ & Ground \\
\hline Crane, Whooping & Grus americana & GRAM & WHCR & 2040 & $\mathrm{R}$ & Ground \\
\hline Creeper, Brown & Certhia americana & CEAM & BRCR & 7260 & B & Woody Upper Canopy \\
\hline Crossbill, Red & Loxia curvirostra & LOCU2 & RECR & 5210 & $\mathrm{R}$ & Woody Upper Canopy \\
\hline Crossbill, White-winged & Loxia leucoptera & LOLE1 & WWCR & 5220 & $\mathrm{R}$ & Woody Upper Canopy \\
\hline Crow, American & Corvus brachyrhynchos & COBR1 & AMCR & 4880 & $\mathrm{R}$ & Woody Upper Canopy, Shrub \\
\hline Crow, Fish & Corvus ossifragus & coos & FICR & 4900 & $\mathrm{R}$ & Woody Upper Canopy, Shrub \\
\hline Crow, Northwestern & Corvus caurinus & COCA2 & NOCR & 4890 & $\mathrm{R}$ & Woody Upper Canopy, Shrub \\
\hline Cuckoo, Black-billed & Coccyzus erythropthalmus & COER & BBCU & 3880 & A & Woody Lower Canopy, Shrub \\
\hline Cuckoo, Mangrove & Coccyzus minor & COMI & MACU & 3860 & $\mathrm{D}$ & Woody Lower Canopy, Shrub \\
\hline Cuckoo, Yellow-billed & Coccyzus americanus & COAM & YBCU & 3870 & A & Woody Lower Canopy, Shrub \\
\hline Curlew, Bristle-thighed & Numenius tahitiensis & NUTA & BTCU & 2680 & $\mathrm{R}$ & Ground \\
\hline Curlew, Eskimo & Numenius borealis & NUBO & ESCU* & 2660 & $\mathrm{R}$ & Ground \\
\hline Curlew, Long-billed & Numenius americanus & NUAM & LBCU & 2640 & A & Ground \\
\hline Dickcissel & Spiza americana & SPAM & DICK & 6040 & A & Ground, Shrub \\
\hline Dipper, American & Cinclus mexicanus & CIME & AMDI & 7010 & $\mathrm{R}$ & Cliff, MMS \\
\hline Dove, Common Ground & Columbina passerina & COPA & COGD & 3200 & $\mathrm{R}$ & Ground, Shrub \\
\hline Dove, Inca & Columbina inca & COIN1 & INDO & 3210 & $\mathrm{R}$ & Shrub, Woody Lower Canopy \\
\hline Dove, Mourning & Zenaida macroura & ZEMA & MODO & 3160 & B & Woody Lower Canopy, Ground \\
\hline Dove, Rock & Columba livia & COLI & RODO* & 3131 & $\mathrm{R}$ & MMS, Cliff \\
\hline Dove, White-tipped & Leptotila verreauxi & LEVE & WTDO & 3180 & $\mathrm{R}$ & Woody Lower Canopy \\
\hline Dove, White-winged & Zenaida asiatica & ZEAS & WWDO & 3190 & $\mathrm{C}$ & Woody Lower Canopy \\
\hline Dovekie & Alle alle & ALAL1 & DOVE & 0340 & $\mathrm{R}$ & Cliff \\
\hline Dowitcher, Long-billed & Limnodromus scolopaceus & LISC & LBDO & 2320 & $\mathrm{R}$ & Ground \\
\hline Dowitcher, Short-billed & Limnodromus griseus & LIGR & SBDO & 2310 & $\mathrm{R}$ & Ground \\
\hline Duck, American Black & Anas rubripes & ANRU & ABDU & 1330 & $\mathrm{R}$ & Ground \\
\hline Duck, Black-bellied Whistling & Dendrocygna autumnalis & DEAU1 & BBWD & 1770 & $\mathrm{R}$ & Snag, Woody Upper Canopy \\
\hline Duck, Fulvous Whistling & Dendrocygna bicolor & DEBI & FUWD & 1780 & $\mathrm{R}$ & Ground, Woody Lower Canopy \\
\hline Duck, Harlequin & Histrionicus histrionicus & HIHI1 & HARD & 1550 & $\mathrm{R}$ & Ground \\
\hline Duck, Masked & Oxyura dominica & OXDO & MADU & 1680 & $\mathrm{R}$ & Ground \\
\hline Duck, Mottled & Anas fulvigula & ANFU & MODU & 1340 & $\mathrm{R}$ & Ground \\
\hline Duck, Ring-necked & Aythya collaris & AYCO & RNDU & 1500 & $\mathrm{R}$ & Ground, Floating \\
\hline
\end{tabular}




\begin{tabular}{|c|c|c|c|c|c|}
\hline $\begin{array}{l}\text { LCTA } \\
\text { CODE }\end{array}$ & NEST TYPE & FOOD TYPE & $\begin{array}{l}\text { FORAGING } \\
\text { SUBSTRATE }\end{array}$ & FORAGING TECHNIQUE & GE NERAL HABITAT \\
\hline PACA2 & Cavity & Insects, Seeds & Foliage, Bark & Foliage Gleaner, Bark Gleaner & Forest, Riparian \\
\hline PASC & Cavity & Insects, Seeds & Foliage, Bark & Foliage Gleaner, Bark Gleaner & Forest \\
\hline PAGA & Cavity & Insects, Seeds & Foliage, Bark & Foliage Gleaner, Bark Gleaner & Forest \\
\hline GYCA1 & None & Carrion, Fish & Ground & Scavenger, High Patrol & Rocky Slope \\
\hline FUAM & Platform & Greens, Aquatic Inverts & Water, Ground & Surface Dips, Ground Gleaner & Freshwater, Swamp/Marsh \\
\hline PHPE1 & Saucer & Fish & Water & Surface Dives & Coastal, Pelagic \\
\hline PHAU & Platform & Fish, Aquatic Inverts & Water & Surface Dives, Surface Dips & Coastal, Freshwater \\
\hline PHCA & Platform & Fish, Aquatic Inverts & Water & Surface Dives & Coastal, Pelagic \\
\hline MOAE & Parasitic & Insects, Seeds & Ground & Ground Gleaner & Shrubland, Cropland \\
\hline MOAT & Parasitic & Insects, Seeds & Ground & Ground Gleaner & Forest Edge, Forest \\
\hline GRCA1 & Saucer & Aquatic Inverts, Insects & Water, Ground & Prober, Ground Gleaner & Swamp/Marsh, Freshwater \\
\hline GRAM & Saucer & Aquatic Inverts, Fish & Water, Ground & Prober, Ground Gleaner & Swamp/Marsh, Freshwater \\
\hline CEAM & Cup & Insects, Nuts & Bark, Air & Bark Gleaner, Hawker & Forest \\
\hline LOCU2 & Cup & Seeds, Fruit & Foliage & Foliage Gleaner & Forest \\
\hline LOLE1 & Cup & Seeds & Foliage, Ground & Foliage Gleaner, Ground Gleaner & Forest \\
\hline COBR1 & Cup & Insects, Seeds & Ground & Scavenger, Ground Gleaner & Forest, Riparian \\
\hline coos & Cup & Aquatic Inverts, Carrion & Ground & Scavenger, Ground Gleaner & Shoreline, Freshwater \\
\hline COCA2 & Cup & Aquatic Inverts, Insects & Ground & Scavenger, Ground Gleaner & Forest, Forest Edge \\
\hline SPAM & Cup & Insects, Seeds & Ground & Ground Gleaner & Grassland, Cropland \\
\hline CIME & Oven & Aquatic Inverts, Fish & Water & Prober & Freshwater, Riparian \\
\hline COPA & Saucer & Seeds, Fruit & Ground & Ground Gleaner & Forest Edge, Urban \\
\hline COIN1 & Saucer & Seeds & Ground & Ground Gleaner & Forest Edge, Shrubland \\
\hline ZEMA & Saucer & Seeds & Ground, Foliage & Ground Gleaner, Foliage Gleaner & Forest Edge, Cropland \\
\hline COLI & Saucer & Seeds & Ground & Ground Gleaner & Urban \\
\hline LEVE & Saucer & Seeds, Fruit & Ground, Foliage & Ground Gleaner, Foliage Gleaner & Riparian, Cropland \\
\hline ZEAS & Saucer & Seeds, Fruit & Ground, Foliage & Ground Gleaner, Foliage Gleaner & Riparian, Shrubland \\
\hline ALAL1 & Crevice & Aquatic Inverts, Fish & Water & Surface Dives & Coastal, Pelagic \\
\hline LISC & Scrape & Aquatic Inverts, Insects & Water & Prober & Tundra, Swamp/Marsh \\
\hline LIGR & Scrape & Aquatic Inverts, Insects & Water & Prober & Tundra, Swamp/Marsh \\
\hline ANRU & Scrape & Aquatic Inverts, Seeds & Water & Dabbler & Swamp/Marsh, Riparian \\
\hline DEAU1 & Cavity & Seeds & Ground & Ground Gleaner & Swamp/Marsh, Riparian \\
\hline DEBI & Saucer & Greens, Seeds & Water, Ground & Surface Dips, Ground Gleaner & Swamp/Marsh \\
\hline HIHI1 & Scrape & Aquatic Inverts & Water & Surface Dives, Dabbler & Freshwater, Coastal \\
\hline OXDO & Scrape & Greens, Insects & Water & Surface Dives & Swamp/Marsh, Freshwater \\
\hline ANFU & Scrape & Aquatic Inverts, Insects & Water & Dabbler & Swamp/Marsh \\
\hline AYCO & Scrape & Greens, Aquatic Inverts & Water & Surface Dives & Swamp/Marsh \\
\hline
\end{tabular}




\begin{tabular}{|c|c|c|c|c|c|c|}
\hline COMMON NAME & SCIENTIFIC NAME & $\begin{array}{l}\text { LCTA } \\
\text { CODE }\end{array}$ & $\begin{array}{l}\text { ALPHA } \\
\text { CODE }\end{array}$ & AOU\# & $\begin{array}{l}\text { NEO } \\
\text { STAT }\end{array}$ & NEST LOCATION \\
\hline Duck, Ruddy & Oxyura jamaicensis & OXJA & RUDU & 1670 & $\mathrm{R}$ & Ground \\
\hline Duck, Tufted & Aythya fuligula & AYFU & TUDU & 1491 & $\mathrm{R}$ & Ground, Bank \\
\hline Duck, Wood & Aix sponsa & AISP & WODU & 1440 & $\mathrm{R}$ & Snag, MMS \\
\hline Dunlin & Calidris alpina & CAAL1 & DUNL & 2430 & $\mathrm{R}$ & Ground \\
\hline Eagle, Bald & Haliaeetus leucocephalus & HALE & BAEA & 3520 & $\mathrm{R}$ & Woody Upper Canopy, Cliff \\
\hline Eagle, Golden & Aquila chrysaetos & $\mathrm{AQCH}$ & GOEA & 3490 & $\mathrm{~B}$ & Cliff, Woody Upper Canopy \\
\hline Eagle, White-tailed & Haliaeetus albicilla & HAAL & WTEA* & 3510 & $\mathrm{R}$ & Woody Upper Canopy, Cliff \\
\hline Egret, Cattle & Bubulcus ibis & BUIB & CAEG & 2001 & $\mathrm{R}$ & Woody Lower Canopy, Shrub \\
\hline Egret, Great & Casmerodius albus & CAAL2 & GREG & 1960 & $\mathrm{R}$ & Woody Upper Canopy, Shrub \\
\hline Egret, Reddish & Egretta rufescens & EGRU & REEG & 1980 & $\mathrm{R}$ & Woody Lower Canopy, Shrub \\
\hline Egret, Snowy & Egretta thula & EGTH & SNEG & 1970 & $\mathrm{R}$ & Woody Lower Canopy, Shrub \\
\hline Eider, Common & Somateria mollissima & SOMO & COEI & 1590 & $\mathrm{R}$ & Ground \\
\hline Eider, King & Somateria spectabilis & SOSP & KIEI & 1620 & $\mathrm{R}$ & Ground \\
\hline Eider, Spectacled & Somateria fischeri & SOFI & SPEI & 1580 & $\mathrm{R}$ & Ground \\
\hline Eider, Steller's & Polysticta stelleri & POST & STEI & 1570 & $\mathrm{R}$ & Ground \\
\hline Falcon, Aplomado & Falco femoralis & FAFE & APFA & 3590 & $\mathrm{R}$ & Woody Lower Canopy, Shrub \\
\hline Falcon, Peregrine & Falco peregrinus & FAPE & PEFA & 3560 & A & Cliff, Woody Upper Canopy \\
\hline Falcon, Prairie & Falco mexicanus & FAME & PRFA & 3550 & B & Cliff, Woody Upper Canopy \\
\hline Fieldfare & Turdus pilaris & TUPI & FIEL & 7612 & $\mathrm{R}$ & Ground, Woody Lower Canopy \\
\hline Finch, Cassin's & Carpodacus cassinii & CACA7 & CAFI & 5180 & B & Woody Upper Canopy \\
\hline Finch, House & Carpodacus mexicanus & CAME2 & $\mathrm{HOFI}$ & 5190 & $\mathrm{R}$ & Woody Upper Canopy, Shrub \\
\hline Finch, Purple & Carpodacus purpureus & CAPU1 & PUFI & 5170 & B & Woody Upper Canopy \\
\hline Finch, Rosy & Leucosticte arctoa & LEAR & $\mathrm{ROFI}^{*}$ & 5240 & $\mathrm{R}$ & Ground, Cliff \\
\hline Flamingo, Greater & Phoenicopterus ruber & PHRU & GREF & 1820 & $\mathrm{R}$ & Ground \\
\hline Flicker, Northern & Colaptes auratus & COAU & NOFL* $^{*}$ & 4120 & B & Snag, Woody Lower Canopy \\
\hline Flycatcher, Acadian & Empidonax virescens & EMVI & ACFL & 4650 & A & Woody Lower Canopy, Shrub \\
\hline Flycatcher, Alder & Empidonax alnorum & EMAL & ALFL & 4661 & A & Shrub, Woody Lower Canopy \\
\hline Flycatcher, Ash-throated & Myiarchus cinerascens & $\mathrm{MYCl}$ & ATFL & 4540 & A & Woody Lower Canopy \\
\hline Flycatcher, Brown-crested & Myiarchus tyrannulus & MYTY & BCFL & 4530 & $\mathrm{C}$ & Woody Lower Canopy, Shrub \\
\hline Flycatcher, Buff-breasted & Empidonax fulvifrons & EMFU & BBFL & 4700 & $\mathrm{C}$ & Woody Upper Canopy \\
\hline Flycatcher, Dusky & Empidonax oberholseri & EMOB & DUFL & 4690 & A & Shrub, Woody Lower Canopy \\
\hline Flycatcher, Dusky-capped & Myiarchus tuberculifer & MYTU & DCFL & 4550 & C & Woody Upper Canopy \\
\hline Flycatcher, Gray & Empidonax wrightii & EMWR & GRFL & 4691 & A & Shrub, Woody Lower Canopy \\
\hline Flycatcher, Great-crested & Myiarchus crinitus & MYCR & GCFL & 4520 & A & Woody Upper Canopy, Snag \\
\hline Flycatcher, Hammond's & Empidonax hammondii & EMHA & HAFL & 4680 & A & Woody Upper Canopy \\
\hline Flycatcher, Least & Empidonax minimus & EMMI1 & LEFL & 4670 & A & Woody Upper Canopy, Shrub \\
\hline Flycatcher, Olive-sided & Contopus borealis & $\mathrm{COBO}$ & OSFL & 4590 & A & Woody Upper Canopy \\
\hline Flycatcher, Scissor-tailed & Tyrannus forficatus & TYFO & STFL & 4430 & A & Woody Upper Canopy, Shrub \\
\hline Flycatcher, Sulphur-bellied & Myiodynastes luteiventris & MYLU & SBFL & 4510 & $\mathrm{C}$ & Woody Upper Canopy \\
\hline Flycatcher, Vermilion & Pyrocephalus rubinus & PYRU & VEFL & 4710 & $A$ & Woody Upper Canopy \\
\hline Flycatcher, Western & Empidonax difficilis & EMDI & WEFL & 4640 & $\mathrm{R}$ & Woody Lower Canopy, Cliff \\
\hline Flycatcher, Willow & Empidonax traillii & EMTR & WIFL & 4660 & $A$ & Shrub, Woody Lower Canopy \\
\hline Flycatcher, Yellow-bellied & Empidonax flaviventris & EMFL & YBFL & 4630 & $A$ & Ground \\
\hline Frigatebird, Magnificent & Fregata magnificens & FRMA & MAFR & 1280 & $\mathrm{R}$ & Shrub, Woody Lower Canopy \\
\hline Fulmar, Northern & Fulmarus glacialis & FUGL & NOFU & 0860 & $\mathrm{R}$ & Cliff, Ground \\
\hline Gadwall & Anas strepera & ANST & GADW & 1350 & $\mathrm{R}$ & Ground \\
\hline Gallinule, Purple & Porphyrula martinica & POMA & PUGA & 2180 & $\mathrm{R}$ & Floating \\
\hline Gannet, Northern & Sula bassanus & SUBA & NOGA & 1170 & $\mathrm{R}$ & Cliff, Ground \\
\hline
\end{tabular}




\begin{tabular}{|c|c|c|c|c|c|}
\hline $\begin{array}{l}\text { LCTA } \\
\text { CODE }\end{array}$ & NEST TYPE & FOOD TYPE & $\begin{array}{l}\text { FORAGING } \\
\text { SUBSTRATE }\end{array}$ & FORAGING TECHNIQUE & GE NERAL HABITAT \\
\hline OXJA & Scrape & Aquatic Inverts, Greens & Water & Surface Dives & Swamp/Marsh, Freshwater \\
\hline AYFU & Burrow & Greens, Insects & Water & Ground Gleaner, Skimmer & Coastal, Freshwater \\
\hline AISP & Cavity & Aquatic Inverts, Seeds & Water & Dabbler & Swamp/Marsh, Riparian \\
\hline CAAL1 & Scrape & Insects, Seeds & Ground, Water & Ground Gleaner, Prober & Coastal, Tundra \\
\hline HALE & Platform & Fish, Birds & Water, Ground & High Patrol, Low Patrol & Freshwater, Coastal \\
\hline $\mathrm{AQCH}$ & Platform & Small Mammals, Birds & Ground & High Patrol, Swooper & Grassland, Forest Edge \\
\hline HAAL & Platform & Fish, Birds & Water, Air & Skimmer, Hawker & Tundra, Coastal \\
\hline BUIB & Platform & Insects, Small Verts & Ground & Ground Gleaner & Swamp/Marsh, Grassland \\
\hline CAAL2 & Platform & Fish, Small Verts & Water & Ambusher & Swamp/Marsh \\
\hline EGRU & Platform & Fish, Aquatic Inverts & Water & Ambusher & Coastal, Swamp/Marsh \\
\hline EGTH & Platform & Aquatic Inverts, Fish & Water & Ambusher & Swamp/Marsh, Freshwater \\
\hline SOMO & Scrape & Aquatic Inverts, Fish & Water & Surface Dives, Dabbler & Tundra, Coastal \\
\hline SOSP & Scrape & Aquatic Inverts & Water & Surface Dives, Dabbler & Tundra, Coastal \\
\hline SOFI & Scrape & Aquatic Inverts, Greens & Water & Dabbler, Surface Dips & Tundra, Coastal \\
\hline POST & Scrape & Aquatic Inverts, Greens & Water & Dabbler, Surface Dips & Tundra, Coastal \\
\hline FAFE & Platform & Birds, Insects & Air & Aerial Pursuit, Hawker & Grassland, Shrubland \\
\hline FAPE & Scrape & Birds & Air & Aerial Pursuit & Forest, Grassland \\
\hline FAME & Scrape, Crevice & Birds, Small Mammals & Air, Ground & Aerial Pursuit, Low Patrol & Grassland, Tundra \\
\hline TUPI & Cup & Insects, Seeds & Ground & Ground Gleaner & Forest \\
\hline CACA7 & Cup & Seeds, Insects & Ground, Foliage & Ground Gleaner, Foliage Gleaner & Forest \\
\hline CAME2 & Cup, Cavity & Seeds, Fruit & Ground, Foliage & Ground Gleaner, Foliage Gleaner & Shrubland, Urban \\
\hline CAPU1 & Cup & Seeds, Insects & Ground, Foliage & Ground Gleaner, Foliage Gleaner & Forest, Forest Edge \\
\hline LEAR & Cup, Crevice & Seeds, Insects & Ground & Ground Gleaner & Rocky Slope, Tundra \\
\hline PHRU & Saucer & Aquatic Inverts, Greens & Water & Prober & Coastal, Swamp/Marsh \\
\hline COAU & Cavity & Insects, Seeds & Ground, Air & Ground Gleaner, Hawker & Forest, Urban \\
\hline EMVI & Cup & Insects, Fruit & Air & Hawker & Riparian, Swamp/Marsh \\
\hline EMAL & Cup & Insects, Fruit & Air & Hawker & Swamp/Marsh, Shrubland \\
\hline $\mathrm{MYCl}$ & Cavity & Insects, Fruit & Air & Hover Gleaner, Hawker & Shrubland, Riparian \\
\hline MYTY & Cavity & Insects, Fruit & Air & Hover Gleaner, Hawker & Shrubland, Desert \\
\hline EMFU & Cup & Insects & Air & Hawker & Forest \\
\hline EMOB & Cup & Insects & Air & Hawker, Hover Gleaner & Forest, Shrubland \\
\hline MYTU & Cavity & Insects, Fruit & Air & Hover Gleaner, Hawker & Riparian, Forest \\
\hline EMWR & Cup & Insects & Air, Ground & Hawker, Ground Gleaner & Shrubland \\
\hline MYCR & Cavity & Insects, Fruit & Air, Ground & Hawker, Ground Gleaner & Forest Edge, Forest \\
\hline EMHA & Cup & Insects & Air & Hawker & Forest \\
\hline EMMI1 & Cup & Insects, Fruit & Air & Hover Gleaner, Hawker & Forest, Urban \\
\hline COBO & Cup & Insects & Air & Hawker & Forest \\
\hline TYFO & Cup & Insects, Fruit & Air, Foliage & Hawker, Foliage Gleaner & Grassland, Shrubland \\
\hline MYLU & Cavity & Insects, Fruit & Air & Hawker & Riparian, Forest \\
\hline PYRU & Cup & Insects & Air & Hawker, Hover \& Pounce & Riparian, Forest Edge \\
\hline EMDI & Cavity & Insects, Fruit & Air & Hawker, Hover Gleaner & Forest, Riparian \\
\hline EMTR & Cup & Insects, Fruit & Air & Hawker, Hover Gleaner & Swamp/Marsh \\
\hline EMFL & Cup & Insects, Fruit & Air & Hawker, Hover Gleaner & Forest, Swamp/Marsh \\
\hline FRMA & Platform & Fish, Aquatic Inverts & Water, Air & Low Patrol, Hawker & Coastal, Pelagic \\
\hline FUGL & Scrape, Saucer & Fish, Aquatic Inverts & Water & Surface Dips, High Dives & Pelagic \\
\hline ANST & Scrape & Greens, Insects & Water & Dabbler, Surface Dives & Swamp/Marsh, Grassland \\
\hline POMA & Platform & Seeds, Fruit & Ground & Ground Gleaner & Swamp/Marsh \\
\hline SUBA & Platform & Fish & Water & High Dives & Coastal, Pelagic \\
\hline
\end{tabular}




\begin{tabular}{|c|c|c|c|c|c|c|}
\hline COMMON NAME & SCIENTIFIC NAME & $\begin{array}{l}\text { LCTA } \\
\text { CODE }\end{array}$ & $\begin{array}{l}\text { ALPHA } \\
\text { CODE }\end{array}$ & AOU\# & $\begin{array}{l}\text { NEO } \\
\text { STAT }\end{array}$ & NEST LOCATION \\
\hline Gnatcatcher, Black-tailed & Polioptila melanura & POME & BTGN & 7520 & $\mathrm{R}$ & Shrub, Woody Lower Canopy \\
\hline Gnatcatcher, Blue-gray & Polioptila caerulea & POCA & BGGN & 7510 & $A$ & Woody Lower Canopy \\
\hline Godwit, Bar-tailed & Limosa lapponica & LILA & BARG & 2500 & $\mathrm{R}$ & Ground \\
\hline Godwit, Hudsonian & Limosa haemastica & LIHA & HUGO & 2510 & $\mathrm{R}$ & Ground \\
\hline Godwit, Marbled & Limosa fedoa & LIFE & MAGO & 2490 & $\mathrm{R}$ & Ground \\
\hline Goldeneye, Barrow's & Bucephala islandica & BUIS & BAGO & 1520 & $\mathrm{R}$ & Snag, Woody Upper Canopy \\
\hline Goldeneye, Common & Bucephala clangula & BUCL & COGO & 1510 & $\mathrm{R}$ & Snag, Woody Upper Canopy \\
\hline Goldfinch, American & Carduelis tristis & CATR & AMGO & 5290 & $\mathrm{~B}$ & Shrub, Woody Lower Canopy \\
\hline Goldfinch, Lawrence's & Carduelis lawrencei & CALA3 & LAGO & 5310 & $\mathrm{~B}$ & Woody Upper Canopy, Shrub \\
\hline Goldfinch, Lesser & Carduelis psaltria & CAPS & LEGO & 5300 & $\mathrm{~B}$ & Woody Lower Canopy, Shrub \\
\hline Goose, Barnacle & Branta leucopsis & BRLE & BRNG & 1750 & $\mathrm{R}$ & Cliff, Ground \\
\hline Goose, Canada & Branta canadensis & BRCA1 & CAGO & 1720 & $\mathrm{R}$ & Ground \\
\hline Goose, Emperor & Chen canagica & CHCA1 & EMGO & 1760 & $\mathrm{R}$ & Ground \\
\hline Goose, Greater White-fronted & Anser albifrons & ANAL & GWFG & 1710 & $\mathrm{R}$ & Ground \\
\hline Goose, Pink-footed & Anser brachyrhynchus & ANBR & PFGO & 1712 & $\mathrm{R}$ & Ground, Cliff \\
\hline Goose, Ross' & Chen rossii & $\mathrm{CHRO}$ & ROGO & 1700 & $\mathrm{R}$ & Ground \\
\hline Goose, Snow & Chen caerulescens & $\mathrm{CHCA}$ & SNGO* & 1690 & $\mathrm{R}$ & Ground \\
\hline Goshawk, Northern & Accipiter gentilis & ACGE & NOGO & 3340 & B & Woody Upper Canopy \\
\hline Grackle, Boat-tailed & Quiscalus major & QUMA & BTGR & 5130 & $\mathrm{R}$ & Woody Lower Canopy, Shrub \\
\hline Grackle, Common & Quiscalus quiscula & QUQU & COGR & 5110 & $\mathrm{R}$ & Woody Lower Canopy \\
\hline Grackle, Great-tailed & Quiscalus mexicanus & QUME & GTGR & 5120 & $\mathrm{R}$ & Woody Lower Canopy, Shrub \\
\hline Grebe, Clark's & Aechmophorus clarkii & $\mathrm{AECL}$ & CLGR & 0011 & $\mathrm{R}$ & Ground \\
\hline Grebe, Eared & Podiceps nigricollis & PONI & EAGR & 0040 & $\mathrm{R}$ & Floating \\
\hline Grebe, Horned & Podiceps auritus & POAU & HOGR & 0030 & $\mathrm{R}$ & Floating \\
\hline Grebe, Least & Tachybaptus dominicus & TADO & LEGR & 0050 & $\mathrm{R}$ & Floating \\
\hline Grebe, Pied-billed & Podilymbus podiceps & POPO & PBGR & 0060 & $\mathrm{R}$ & Floating \\
\hline Grebe, Red-necked & Podiceps grisegena & POGR & RNGR & 0020 & $\mathrm{R}$ & Floating \\
\hline Grebe, Western & Aechmophorus occidentalis & AEOC & WEGR & 0010 & $\mathrm{R}$ & Floating \\
\hline Grosbeak, Black-headed & Pheucticus melanocephalus & PHME1 & BHGR & 5960 & A & Woody Lower Canopy, Shrub \\
\hline Grosbeak, Blue & Guiraca caerulea & GUCA & BLGR & 5970 & A & Shrub, Woody Lower Canopy \\
\hline Grosbeak, Evening & Coccothraustes vespertinus & COVE & EVGR & 5140 & $\mathrm{R}$ & Woody Upper Canopy \\
\hline Grosbeak, Pine & Pinicola enucleator & PIEN & PIGR & 5150 & $\mathrm{R}$ & Woody Lower Canopy, Shrub \\
\hline Grosbeak, Rose-breasted & Pheucticus ludovicianus & PHLU & RBGR & 5950 & A & Woody Lower Canopy, Shrub \\
\hline Grouse, Blue & Dendragapus obscurus & DEOB & $\mathrm{BLGS}^{*}$ & 2970 & $\mathrm{R}$ & Ground \\
\hline Grouse, Ruffed & Bonasa umbellus & BOUM & $\mathrm{RUGR}^{\star}$ & 3000 & $\mathrm{R}$ & Ground \\
\hline Grouse, Sage & Centrocercus urophasianus & CEUR & SAGR* & 3090 & $\mathrm{R}$ & Ground \\
\hline Grouse, Sharp-tailed & Tympanuchus phasianellus & TYPH & STGR* & 3080 & $\mathrm{R}$ & Ground \\
\hline Grouse, Spruce & Dendragapus canadensis & DECA & SPGR* & 2980 & $\mathrm{R}$ & Ground \\
\hline Guillemot, Black & Cepphus grylle & CEGR & BLGU & 0270 & $\mathrm{R}$ & Cliff, Ground \\
\hline Guillemot, Pigeon & Cepphus columba & CECO1 & PIGU & 0290 & $\mathrm{R}$ & Cliff \\
\hline Gull, Bonaparte's & Larus philadelphia & $\mathrm{LAPH}$ & BOGU & 0600 & $\mathrm{R}$ & Woody Lower Canopy, Ground \\
\hline Gull, California & Larus californicus & LACA1 & CAGU & 0530 & $\mathrm{R}$ & Ground \\
\hline Gull, Common Black-headed & Larus ridibundus & LARI & $\mathrm{CBHG}$ & 0551 & $\mathrm{R}$ & Ground \\
\hline Gull, Franklin's & Larus pipixcan & LAPI & FRGU & 0590 & $\mathrm{R}$ & Floating \\
\hline Gull, Glaucous & Larus hyperboreus & LAHY & GLGU & 0420 & $\mathrm{R}$ & Cliff, Ground \\
\hline Gull, Glaucous-winged & Larus glaucescens & LAGL & GWGU & 0440 & $\mathrm{R}$ & Cliff, Ground \\
\hline Gull, Great Black-backed & Larus marinus & LAMA & GBBG & 0470 & $\mathrm{R}$ & Ground \\
\hline Gull, Heermann's & Larus heermanni & LAHE & HEEG & 0570 & $\mathrm{R}$ & Ground \\
\hline
\end{tabular}




\begin{tabular}{|c|c|c|c|c|c|}
\hline $\begin{array}{l}\text { LCTA } \\
\text { CODE }\end{array}$ & NEST TYPE & FOOD TYPE & $\begin{array}{l}\text { FORAGING } \\
\text { SUBSTRATE }\end{array}$ & FORAGING TECHNIQUE & GE NERAL HABITAT \\
\hline POME & Cup & Insects, Seeds & Foliage, Air & Foliage Gleaner, Hover Gleaner & Shrubland \\
\hline POCA & Cup & Insects & Foliage, Air & Foliage Gleaner, Hover Gleaner & Forest, Shrubland \\
\hline LILA & Scrape & Aquatic Inverts, Insects & Water & Prober & Tundra, Coastal \\
\hline LIHA & Scrape & Insects, Aquatic Inverts & Water & Prober & Tundra \\
\hline LIFE & Scrape & Aquatic Inverts, Insects & Water, Ground & Prober, Ground Gleaner & Grassland, Swamp/Marsh \\
\hline BUIS & Cavity & Aquatic Inverts & Water & Surface Dives & Swamp/Marsh, Freshwater \\
\hline BUCL & Cavity & Aquatic Inverts, Fish & Water & Surface Dives & Freshwater, Swamp/Marsh \\
\hline CATR & Cup & Seeds, Insects & Foliage, Ground & Foliage Gleaner, Ground Gleaner & Grassland, Riparian \\
\hline CALA3 & Cup & Seeds, Insects & Foliage, Ground & Foliage Gleaner, Ground Gleaner & Riparian, Shrubland \\
\hline CAPS & Cup & Seeds, Insects & Foliage & Foliage Gleaner & Forest Edge, Grassland \\
\hline BRLE & Scrape & Greens, Seeds & Ground & Ground Gleaner & Tundra, Coastal \\
\hline BRCA1 & Scrape & Greens, Aquatic Inverts & Water & Surface Dips, Dabbler & Swamp/Marsh, Freshwater \\
\hline CHCA1 & Scrape & Greens, Aquatic Inverts & Ground, Water & Ground Gleaner, Dabbler & Swamp/Marsh, Tundra \\
\hline ANAL & Scrape & Greens, Aquatic Inverts & Water & Surface Dips, Dabbler & Freshwater, Swamp/Marsh \\
\hline ANBR & Scrape & Grain, Greens & Ground & Ground Gleaner & Tundra, Coastal \\
\hline $\mathrm{CHRO}$ & Scrape & Greens, Aquatic Inverts & Water & Surface Dips, Dabbler & Freshwater, Tundra \\
\hline $\mathrm{CHCA}$ & Scrape & Greens, Aquatic Inverts & Water & Surface Dips, Dabbler & Tundra, Freshwater \\
\hline ACGE & Platform & Birds, Small Mammals & Air, Ground & Aerial Pursuit, Low Patrol & Forest \\
\hline QUMA & Cup & Insects, Small Verts & Ground, Air & Ground Gleaner, Hawker & Swamp/Marsh, Cropland \\
\hline QUQU & Cup, Cavity & Insects, Aquatic Inverts & Ground, Foliage & Ground Gleaner, Foliage Gleaner & Grassland, Urban \\
\hline QUME & Cup & Insects, Small Verts & Ground & Ground Gleaner & Grassland, Swamp/Marsh \\
\hline AECL & Platform & Fish, Aquatic Inverts & Water & Surface Dives & Freshwater, Swamp/Marsh \\
\hline PONI & Platform & Aquatic Inverts, Fish & Water & Surface Dives & Swamp/Marsh, Freshwater \\
\hline POAU & Platform & Aquatic Inverts, Fish & Water & Surface Dives & Swamp/Marsh, Freshwater \\
\hline TADO & Platform & Aquatic Inverts, Fish & Water & Surface Dives & Freshwater, Swamp/Marsh \\
\hline POPO & Platform & Aquatic Inverts, Fish & Water & Surface Dives & Swamp/Marsh, Riparian \\
\hline POGR & Platform & Aquatic Inverts, Fish & Water & Surface Dives & Freshwater, Swamp/Marsh \\
\hline AEOC & Platform & Fish, Aquatic Inverts & Water & Surface Dives & Swamp/Marsh, Freshwater \\
\hline PHME1 & Cup & Insects, Seeds & Foliage & Foliage Gleaner & Riparian, Forest Edge \\
\hline GUCA & Cup & Insects, Seeds & Ground, Foliage & Ground Gleaner, Foliage Gleaner & Riparian, Forest Edge \\
\hline COVE & Cup & Seeds, Fruit & Ground, Foliage & Ground Gleaner, Foliage Gleaner & Forest, Urban \\
\hline PIEN & Cup & Seeds, Fruit & Foliage, Ground & Foliage Gleaner, Ground Gleaner & Forest, Forest Edge \\
\hline PHLU & Cup & Insects, Seeds & Foliage, Air & Foliage Gleaner, Hover Gleaner & Forest, Shrubland \\
\hline DEOB & Scrape & Greens, Seeds & Ground, Foliage & Ground Gleaner, Foliage Gleaner & Forest \\
\hline BOUM & Scrape & Greens, Seeds & Foliage, Ground & Foliage Browser, Ground Gleaner & Forest \\
\hline CEUR & Scrape & Greens, Insects & Foliage, Ground & Foliage Browser, Ground Gleaner & Shrubland \\
\hline TYPH & Scrape & Greens, Seeds & Ground, Foliage & Ground Gleaner, Foliage Browser & Grassland, Shrubland \\
\hline DECA & Scrape & Greens, Fruit & Foliage, Ground & Foliage Browser, Ground Gleaner & Forest \\
\hline CEGR & Crevice & Fish, Aquatic Inverts & Water & Surface Dives & Coastal, Pelagic \\
\hline CECO1 & Crevice, Burrow & Fish, Aquatic Inverts & Water & Surface Dives & Coastal, Pelagic \\
\hline LAPH & Saucer & Insects, Aquatic Inverts & Ground, Water & Scavenger, Surface Dives & Shoreline, Freshwater \\
\hline LACA1 & Scrape & Insects, Small Verts & Water & Scavenger, Surface Dives & Coastal, Freshwater \\
\hline LARI & Scrape & Insects & Water & Scavenger, Ground Gleaner & Coastal, Freshwater \\
\hline LAPI & Platform & Insects & Water & Scavenger, Ground Gleaner & Freshwater, Cropland \\
\hline LAHY & Saucer & Fish, Aquatic Inverts & Water & Scavenger, Low Patrol & Coastal \\
\hline LAGL & Saucer & Aquatic Inverts, Fish & Water, Ground & Scavenger, Ground Gleaner & Coastal \\
\hline LAMA & Saucer & Birds, Fish & Ground, Water & Scavenger, Ground Gleaner & Coastal \\
\hline LAHE & Scrape, Saucer & Fish, Aquatic Inverts & Water & Scavenger, High Dives & Coastal \\
\hline
\end{tabular}




\begin{tabular}{|c|c|c|c|c|c|c|}
\hline COMMON NAME & SCIENTIFIC NAME & $\begin{array}{l}\text { LCTA } \\
\text { CODE }\end{array}$ & $\begin{array}{l}\text { ALPHA } \\
\text { CODE }\end{array}$ & AOU\# & $\begin{array}{l}\text { NEO } \\
\text { STAT }\end{array}$ & NEST LOCATION \\
\hline Gull, Herring & Larus argentatus & LAAR & HERG & 0510 & $\mathrm{R}$ & Ground, Cliff \\
\hline Gull, Iceland & Larus glaucoides & LAGL1 & ICGU & 0430 & $\mathrm{R}$ & Cliff, Ground \\
\hline Gull, Ivory & Pagophila eburnea & PAEB & IVGU & 0390 & $\mathrm{R}$ & Ground, Cliff \\
\hline Gull, Laughing & Larus atricilla & LAAT & LAGU & 0580 & $\mathrm{R}$ & Ground \\
\hline Gull, Lesser Black-backed & Larus fuscus & LAFU & LBBG & 0500 & $\mathrm{R}$ & Ground \\
\hline Gull, Little & Larus minutus & LAMI & LIGU & 0601 & $\mathrm{R}$ & Ground \\
\hline Gull, Mew & Larus canus & LACA2 & MEGU & 0550 & $\mathrm{R}$ & Ground, Floating \\
\hline Gull, Ring-billed & Larus delawarensis & LADE1 & RBGU & 0540 & $\mathrm{R}$ & Ground \\
\hline Gull, Ross' & Rhodostethia rosea & $\mathrm{RHRO}$ & ROGU & 0610 & $\mathrm{R}$ & Ground \\
\hline Gull, Sabine's & Xema sabini & XESA & SAGU & 0620 & $\mathrm{R}$ & Ground \\
\hline Gull, Thayer's & Larus thayeri & LATH & THGU & 0431 & $\mathrm{R}$ & Cliff \\
\hline Gull, Western & Larus occidentalis & LAOC & WEGU & 0490 & $\mathrm{R}$ & Cliff, Ground \\
\hline Gull, Yellow-footed & Larus livens & LALI & YFGU & 0491 & $\mathrm{R}$ & Ground \\
\hline Gyrfalcon & Falco rusticolus & FARU & GYRF & 3540 & $\mathrm{R}$ & Cliff, Woody Upper Canopy \\
\hline Harrier, Northern & Circus cyaneus & CICY & $\mathrm{NOHA}$ & 3310 & $\mathrm{~B}$ & Ground, Shrub \\
\hline Hawk, Broad-winged & Buteo platypterus & BUPL & BWHA & 3430 & $A$ & Woody Upper Canopy \\
\hline Hawk, Common Black & Buteogallus anthracinus & BUAN & $\mathrm{CBHA}$ & 3450 & C & Woody Upper Canopy \\
\hline Hawk, Cooper's & Accipiter cooperii & ACCO & $\mathrm{COHA}$ & 3330 & $\mathrm{~B}$ & Woody Upper Canopy \\
\hline Hawk, Ferruginous & Buteo regalis & BURE1 & FEHA & 3480 & $\mathrm{~B}$ & Woody Upper Canopy, Cliff \\
\hline Hawk, Gray & Buteo nitidus & BUNI & GRHA & 3460 & $\mathrm{C}$ & Woody Upper Canopy \\
\hline Hawk, Harris' & Parabuteo unicinctus & PAUN & $\mathrm{HRSH}$ & 3350 & $\mathrm{R}$ & Woody Upper Canopy, Shrub \\
\hline Hawk, Red-shouldered & Buteo lineatus & BULI & RSHA & 3390 & $\mathrm{~B}$ & Woody Upper Canopy \\
\hline Hawk, Red-tailed & Buteo jamaicensis & BUJA & RTHA & 3370 & B & Woody Upper Canopy, Cliff \\
\hline Hawk, Rough-legged & Buteo lagopus & BULA & RLHA & 3470 & $\mathrm{R}$ & Cliff, Woody Upper Canopy \\
\hline Hawk, Sharp-shinned & Accipiter striatus & ACST & SSHA & 3320 & B & Woody Upper Canopy \\
\hline Hawk, Short-tailed & Buteo brachyurus & BUBR & STHA & 3440 & $\mathrm{R}$ & Woody Upper Canopy \\
\hline Hawk, Swainson's & Buteo swainsoni & BUSW & SWHA & 3420 & $A$ & Woody Upper Canopy, Cliff \\
\hline Hawk, White-tailed & Buteo albicaudatus & BUAL2 & WTHA & 3410 & $\mathrm{R}$ & Woody Lower Canopy, Shrub \\
\hline Hawk, Zone-tailed & Buteo albonotatus & BUAL3 & ZTHA & 3400 & C & Woody Upper Canopy \\
\hline Heron, Black-crowned Night & Nycticorax nycticorax & NYNY & $\mathrm{BCNH}$ & 2020 & $\mathrm{R}$ & Woody Lower Canopy, Ground \\
\hline Heron, Great Blue & Ardea herodias & ARHE & GTBH & 1940 & $\mathrm{R}$ & Woody Upper Canopy \\
\hline Heron, Green-backed & Butorides striatus & BUST & GNBH & 2010 & $\mathrm{R}$ & Woody Lower Canopy, Shrub \\
\hline Heron, Little Blue & Egretta caerulea & EGCA & LBHE & 2000 & $\mathrm{R}$ & Woody Upper Canopy, Shrub \\
\hline Heron, Tricolored & Egretta tricolor & EGTR & TRHE & 1990 & $\mathrm{R}$ & Woody Lower Canopy, Shrub \\
\hline Heron, Yellow-crowned Night & Nycticorax violaceus & NYVI & YCNH & 2030 & $\mathrm{R}$ & Woody Upper Canopy, Shrub \\
\hline Hummingbird, Allen's & Selasphorus sasin & SESA & ALHU & 4340 & $A$ & Woody Lower Canopy, Shrub \\
\hline Hummingbird, Anna's & Calypte anna & CAAN & ANHU & 4310 & $\mathrm{~B}$ & Woody Lower Canopy, Shrub \\
\hline Hummingbird, Berylline & Amazilia beryllina & AMBE & BEHU & 4381 & $\mathrm{R}$ & Woody Upper Canopy \\
\hline Hummingbird, Black-chinned & Archilochus alexandri & ARAL1 & $\mathrm{BCHU}$ & 4290 & $A$ & Woody Lower Canopy \\
\hline Hummingbird, Blue-throated & Lampornis clemenciae & LACL & BLUH & 4270 & $\mathrm{C}$ & Ground, Woody Lower Canopy \\
\hline Hummingbird, Broad-billed & Cynanthus latirostris & CYLA & BBLH & 4410 & $\mathrm{C}$ & Woody Lower Canopy, Shrub \\
\hline Hummingbird, Broad-tailed & Selasphorus platycercus & SEPL & BTLH & 4320 & $A$ & Woody Lower Canopy \\
\hline Hummingbird, Buff-bellied & Amazilia yucatanensis & AMYU & BUFH & 4390 & $\mathrm{C}$ & Shrub, Woody Lower Canopy \\
\hline Hummingbird, Calliope & Stellula calliope & STCA & CAHU & 4360 & $A$ & Woody Upper Canopy, Shrub \\
\hline Hummingbird, Costa's & Calypte costae & CACO & $\mathrm{COHU}$ & 4300 & $A$ & Shrub, Woody Lower Canopy \\
\hline Hummingbird, Lucifer & Calothorax lucifer & CALU & LUHU & 4370 & $\mathrm{C}$ & Shrub, Woody Lower Canopy \\
\hline Hummingbird, Magnificent & Eugenes fulgens & EUFU & MAHU & 4260 & $\mathrm{C}$ & Woody Upper Canopy \\
\hline Hummingbird, Ruby-throated & Archilochus colubris & ARCO & RTHU & 4280 & $A$ & Woody Lower Canopy \\
\hline
\end{tabular}




\begin{tabular}{|c|c|c|c|c|c|}
\hline $\begin{array}{l}\text { LCTA } \\
\text { CODE }\end{array}$ & NEST TYPE & FOOD TYPE & $\begin{array}{l}\text { FORAGING } \\
\text { SUBSTRATE }\end{array}$ & FORAGING TECHNIQUE & GENERAL HABITAT \\
\hline LAAR & Saucer & Seeds, Birds & Water, Ground & Scavenger, Ground Gleaner & Coastal, Freshwater \\
\hline LAGL1 & Saucer & Fish, Aquatic Inverts & Water & Scavenger, High Dives & Coastal \\
\hline PAEB & Saucer, Scrape & Fish, Aquatic Inverts & Water, Ground & Scavenger, High Dives & Coastal \\
\hline LAAT & Scrape, Saucer & Aquatic Inverts & Ground, Water & Scavenger, Ground Gleaner & Swamp/Marsh \\
\hline LAFU & Saucer & Fish, Aquatic Inverts & Water & Scavenger & Coastal \\
\hline LACA2 & Scrape, Cup & Insects & Ground, Water & Scavenger, Ground Gleaner & Coastal, Tundra \\
\hline LADE1 & Saucer & Fish, Insects & Ground, Water & Scavenger, Ground Gleaner & Freshwater, Coastal \\
\hline RHRO & Scrape & Insects, Fish & Water, Ground & Surface Dips, Ground Gleaner & Coastal, Tundra \\
\hline XESA & Scrape & Fish, Insects & Ground, Water & Scavenger, Ground Gleaner & Tundra, Freshwater \\
\hline LATH & Saucer & Fish, Fruit & Water, Ground & Scavenger, Low Patrol & Coastal \\
\hline $\mathrm{CICY}$ & Platform & Small Mammals, Small Verts & Ground, Air & Low Patrol & Grassland, Swamp/Marsh \\
\hline BUPL & Platform & Small Mammals, Birds & Ground, Air & Swooper & Forest \\
\hline BUAN & Platform & Small Verts, Fish & Ground, Water & Swooper & Swamp/Marsh, Riparian \\
\hline ACCO & Platform & Birds, Small Mammals & Air, Ground & Aerial Pursuit, Low Patrol & Forest, Riparian \\
\hline BURE1 & Platform & Small Mammals, Birds & Ground & Hover \& Pounce, Swooper & Grassland \\
\hline BUNI & Platform & Small Verts, Birds & Ground & Swooper, Low Patrol & Riparian, Forest \\
\hline PAUN & Platform & Small Mammals, Small Verts & Ground & Low Patrol, Swooper & Grassland, Shrubland \\
\hline BULI & Platform & Small Mammals, Small Verts & Ground & Low Patrol, High Patrol & Riparian, Swamp/Marsh \\
\hline BUJA & Platform & Small Mammals, Birds & Ground & High Patrol, Swooper & Forest, Grassland \\
\hline BULA & Platform & Small Mammals, Insects & Ground & Hover \& Pounce, Swooper & Forest, Tundra \\
\hline ARHE & Platform & Fish, Aquatic Inverts & Water & Ambusher & Swamp/Marsh, Freshwater \\
\hline BUST & Platform & Fish, Insects & Water & Ambusher & Swamp/Marsh, Freshwater \\
\hline EGCA & Platform & Fish, Small Verts & Water & Ambusher & Swamp/Marsh, Freshwater \\
\hline EGTR & Platform & Fish, Small Verts & Water & Ambusher & Swamp/Marsh, Freshwater \\
\hline NYVI & Platform & Aquatic Inverts, Insects & Water, Ground & Ambusher, Ground Gleaner & Swamp/Marsh, Freshwater \\
\hline SESA & Cup & Nectar, Insects & Air & Hover Gleaner, Hawker & Shrubland, Forest \\
\hline CAAN & Cup & Nectar, Insects & Air & Hover Gleaner, Hawker & Forest, Shrubland \\
\hline AMBE & Cup & Nectar, Insects & Air & Hover Gleaner & Forest \\
\hline ARAL1 & Cup & Nectar, Insects & Air & Hover Gleaner, Hawker & Forest, Shrubland \\
\hline LACL & Cup & Insects, Nectar & Air & Hover Gleaner, Hawker & Forest, Riparian \\
\hline CYLA & Cup & Nectar, Insects & Air & Hover Gleaner & Shrubland, Riparian \\
\hline SEPL & Cup & Nectar, Insects & Air & Hover Gleaner, Hawker & Forest, Shrubland \\
\hline AMYU & Cup & Nectar, Insects & Air & Hover Gleaner & Forest, Shrubland \\
\hline STCA & Cup & Nectar, Insects & Air & Hover Gleaner, Hawker & Forest, Shrubland \\
\hline CACO & Cup & Nectar, Insects & Air & Hover Gleaner & Shrubland \\
\hline CALU & Cup & Insects, Nectar & Air & Hover Gleaner & Shrubland \\
\hline EUFU & Cup & Nectar, Insects & Air & Hover Gleaner & Forest \\
\hline ARCO & Cup & Nectar, Insects & Air & Hover Gleaner & Forest, Urban \\
\hline
\end{tabular}




\begin{tabular}{|c|c|c|c|c|c|c|}
\hline COMMON NAME & SCIENTIFIC NAME & $\begin{array}{l}\text { LCTA } \\
\text { CODE }\end{array}$ & $\begin{array}{l}\text { ALPHA } \\
\text { CODE }\end{array}$ & AOU\# & $\begin{array}{l}\text { NEO } \\
\text { STAT }\end{array}$ & NEST LOCATION \\
\hline Hummingbird, Rufous & Selasphorus rufus & SERU & RUHU & 4330 & $\mathrm{~A}$ & Woody Lower Canopy \\
\hline Hummingbird, Violet-crowned & Amazilia violiceps & AMVI & VCHU & 4391 & $\mathrm{C}$ & Woody Upper Canopy \\
\hline Hummingbird, White-eared & Hylocharis leucotis & HYLE & WEHU & 4401 & $\mathrm{R}$ & Shrub, Woody Lower Canopy \\
\hline Ibis, Glossy & Plegadis falcinellus & PLFA & GLIB & 1860 & $\mathrm{R}$ & Ground, Shrub \\
\hline Ibis, Scarlet & Eudocimus ruber & EURU & SCIB & 1850 & $\mathrm{R}$ & Woody Upper Canopy \\
\hline Ibis, White & Eudocimus albus & EUAL & WHIB & 1840 & $\mathrm{R}$ & Woody Lower Canopy \\
\hline Ibis, White-faced & Plegadis chihi & $\mathrm{PLCH}$ & WFIB & 1870 & $\mathrm{R}$ & Ground, Shrub \\
\hline Jacana, Northern & Jacana spinosa & JASP & NOJA & 2880 & $\mathrm{R}$ & Floating \\
\hline Jaeger, Long-tailed & Stercorarius longicaudus & STLO1 & LTJA & 0380 & $\mathrm{R}$ & Ground \\
\hline Jaeger, Parasitic & Stercorarius parasiticus & STPA & PAJA & 0370 & $\mathrm{R}$ & Ground \\
\hline Jaeger, Pomarine & Stercorarius pomarinus & STPO & POJA & 0360 & $\mathrm{R}$ & Ground \\
\hline Jay, Blue & Cyanocitta cristata & CYCR & BLJA & 4770 & $\mathrm{R}$ & Woody Lower Canopy \\
\hline Jay, Brown & Cyanocorax morio & CYMO & BRJA & 4832 & $\mathrm{R}$ & Woody Lower Canopy, Shrub \\
\hline Jay, Gray & Perisoreus canadensis & PECA & GRAJ & 4840 & $\mathrm{R}$ & Woody Lower Canopy \\
\hline Jay, Gray-breasted & Aphelocoma ultramarina & APUL & GBJA & 4820 & $\mathrm{R}$ & Woody Lower Canopy \\
\hline Jay, Green & Cyanocorax yncas & CYYN & GREJ & 4830 & $\mathrm{R}$ & Woody Lower Canopy, Shrub \\
\hline Jay, Pinyon & Gymnorhinus cyanocephalus & GYCY & PIJA & 4920 & $\mathrm{R}$ & Woody Lower Canopy \\
\hline Jay, Scrub & Aphelocoma coerulescens & APCO & SCJA & 4810 & $\mathrm{R}$ & Woody Lower Canopy, Shrub \\
\hline Jay, Steller's & Cyanocitta stelleri & CYST & STJA & 4780 & $\mathrm{R}$ & Woody Lower Canopy \\
\hline Junco, Dark-eyed & Junco hyemalis & JUHY & DEJU* & 5670 & $\mathrm{~B}$ & Ground, Bank \\
\hline Junco, Yellow-eyed & Junco phaeonotus & JUPH & YEJU & 5700 & $\mathrm{R}$ & Ground, Shrub \\
\hline Kestrel, American & Falco sparverius & FASP & AMKE & 3600 & $\mathrm{~B}$ & Snag, Cliff \\
\hline Killdeer & Charadrius vociferus & $\mathrm{CHVO}$ & KILL & 2730 & $\mathrm{~B}$ & Ground \\
\hline Kingbird, Cassin's & Tyrannus vociferans & TYVO & CAKI & 4480 & $A$ & Woody Upper Canopy \\
\hline Kingbird, Couch's & Tyrannus couchii & TYCO & $\mathrm{COKI}$ & 4461 & $\mathrm{C}$ & Woody Lower Canopy \\
\hline Kingbird, Eastern & Tyrannus tyrannus & TYTY & EAKI & 4440 & $A$ & Woody Lower Canopy, Shrub \\
\hline Kingbird, Gray & Tyrannus dominicensis & TYDO & GRAK & 4450 & $\mathrm{D}$ & Woody Lower Canopy, Shrub \\
\hline Kingbird, Thick-billed & Tyrannus crassirostris & TYCR & TBKI & 4451 & C & Woody Upper Canopy \\
\hline Kingbird, Tropical & Tyrannus melancholicus & TYME & TRKI & 4460 & C & Woody Lower Canopy \\
\hline Kingbird, Western & Tyrannus verticalis & TYVE & WEKI & 4470 & A & Woody Upper Canopy, Shrub \\
\hline Kingfisher, Belted & Ceryle alcyon & CEAL & BEKI & 3900 & B & Bank, Snag \\
\hline Kingfisher, Green & Chloroceryle americana & CHAM & GKIN & 3910 & C & Bank \\
\hline Kingfisher, Ringed & Ceryle torquata & CETO & $\mathrm{RIKI}$ & 3901 & $\mathrm{R}$ & Bank \\
\hline Kinglet, Golden-crowned & Regulus satrapa & RESA & GCKI & 7480 & $\mathrm{R}$ & Woody Upper Canopy \\
\hline Kinglet, Ruby-crowned & Regulus calendula & RECA & $\mathrm{RCKI}$ & 7490 & B & Woody Upper Canopy \\
\hline Kiskadee, Great & Pitangus sulphuratus & PISU1 & GKIS & 4490 & $\mathrm{R}$ & Woody Lower Canopy, Shrub \\
\hline $\begin{array}{l}\text { Kite, American Swallow- } \\
\text { tailed }\end{array}$ & Elanoides forficatus & ELFO & ASTK & 3270 & A & Woody Upper Canopy \\
\hline Kite, Black-shouldered & Elanus caeruleus & ELCA & BSKI & 3280 & $\mathrm{R}$ & Woody Upper Canopy \\
\hline Kite, Hook-billed & Chondrohierax uncinatus & CHUN & HBKI & 3271 & $\mathrm{R}$ & Woody Lower Canopy \\
\hline Kite, Mississippi & Ictinia mississippiensis & ICMI & MIKI & 3290 & $A$ & Woody Upper Canopy \\
\hline Kite, Snail & Rostrhamus sociabilis & ROSO & SNKI & 3300 & $\mathrm{R}$ & Woody Lower Canopy, Shrub \\
\hline Kittiwake, Black-legged & Rissa tridactyla & RITR & BLKI & 0400 & $\mathrm{R}$ & Cliff \\
\hline Kittiwake, Red-legged & Rissa brevirostris & RIBR & RLKI & 0410 & $\mathrm{R}$ & Cliff \\
\hline Knot, Red & Calidris canutus & CACA & REKN & 2340 & $\mathrm{R}$ & Ground \\
\hline Lark, Horned & Eremophila alpestris & ERAL & HOLA & 4740 & $B$ & Ground \\
\hline Limpkin & Aramus guarauna & ARGU & LIMP & 2070 & $\mathrm{R}$ & Ground, Shrub \\
\hline Longspur, Chestnut-collared & Calcarius ornatus & CAOR & CCLO & 5380 & B & Ground \\
\hline Longspur, Lapland & Calcarius lapponicus & CALA & LALO & 5360 & $\mathrm{R}$ & Ground \\
\hline
\end{tabular}




\begin{tabular}{|c|c|c|c|c|c|}
\hline $\begin{array}{l}\text { LCTA } \\
\text { CODE }\end{array}$ & NEST TYPE & FOOD TYPE & $\begin{array}{l}\text { FORAGING } \\
\text { SUBSTRATE }\end{array}$ & FORAGING TECHNIQUE & GENERAL HABITAT \\
\hline SERU & Cup & Nectar, Insects & Air & Hover Gleaner, Hawker & Forest, Shrubland \\
\hline AMVI & Cup & Nectar, Insects & Air & Hover Gleaner & Forest, Riparian \\
\hline HYLE & Cup & Nectar, Insects & Air & Hover Gleaner, Hawker & Forest, Shrubland \\
\hline PLFA & Platform & Aquatic Inverts, Insects & Water, Ground & Prober, Ground Gleaner & Swamp/Marsh \\
\hline EURU & Platform & Aquatic Inverts, Fish & Water & Prober & Swamp/Marsh, Freshwater \\
\hline PLCH & Platform & Aquatic Inverts, Insects & Water, Ground & Prober, Ground Gleaner & Swamp/Marsh, Freshwater \\
\hline JASP & Platform & Insects & Foliage, Ground & Foliage Gleaner, Ground Gleaner & Swamp/Marsh, Freshwater \\
\hline STLO1 & Scrape & Small Mammals, Birds & Ground, Air & Hover \& Pounce, Piracy & Grassland, Tundra \\
\hline STPA & Scrape & Birds, Small Mammals & Ground, Air & Hover \& Pounce, Piracy & Shrubland, Tundra \\
\hline STPO & Scrape & Small Mammals, Birds & Ground, Air & Low Patrol, Piracy & Swamp/Marsh, Tundra \\
\hline APUL & Cup & Nuts & Ground & Ground Gleaner & Forest, Shrubland \\
\hline CYYN & Cup & Insects, Small Verts & Foliage, Ground & Foliage Gleaner, Ground Gleaner & Shrubland, Forest \\
\hline GYCY & Cup & Nuts, Seeds & Ground, Foliage & Ground Gleaner, Foliage Gleaner & Shrubland, Forest \\
\hline APCO & Cup & Insects, Nuts & Ground & Ground Gleaner & Shrubland, Forest \\
\hline CYST & Cup & Nuts, Seeds & Ground, Foliage & Ground Gleaner, Foliage Gleaner & Forest \\
\hline JUHY & Cup, Cavity & Seeds, Insects & Ground, Air & Ground Gleaner, Hawker & Forest, Forest Edge \\
\hline JUPH & Cup, Cavity & Insects, Seeds & Ground, Foliage & Ground Gleaner, Foliage Gleaner & Forest, Shrubland \\
\hline FASP & Cavity & Insects, Small Verts & Ground, Air & Hover \& Pounce, Hawker & Grassland, Cropland \\
\hline CHVO & Scrape & Insects & Ground & Ground Gleaner & Shoreline, Freshwater \\
\hline TYVO & Cup & Insects, Fruit & Air, Ground & Hawker, Hover \& Pounce & Forest, Shrubland \\
\hline CEAL & Burrow, Cavity & Fish, Aquatic Inverts & Water & High Dives & Shoreline, Freshwater \\
\hline CHAM & Burrow & Fish & Water & High Dives & Freshwater, Swamp/Marsh \\
\hline CETO & Burrow & Fish & Water & High Dives & Freshwater \\
\hline RESA & Pendant & Insects, Fruit & Bark, Air & Foliage Gleaner, Hover Gleaner & Forest \\
\hline RECA & Pendant & Insects, Tree Sap & Foliage, Air & Foliage Gleaner, Hover Gleaner & Forest \\
\hline PISU1 & Sphere & Insects, Fish & Air, Water & Hawker, High Dives & Forest, Riparian \\
\hline ELFO & Platform & Insects, Small Verts & Air, Ground & Hawker, Swooper & Forest, Swamp/Marsh \\
\hline ELCA & Platform & Small Mammals, Insects & Ground & Hover \& Pounce & Grassland, Riparian \\
\hline CHUN & Platform & Aquatic Inverts, Insects & Ground, Bark & Swooper, Bark Gleaner & Swamp/Marsh, Forest \\
\hline ICMI & Saucer & Insects, Small Verts & Air, Ground & Hawker, High Patrol & Riparian, Forest Edge \\
\hline ROSO & Platform & Aquatic Inverts & Water & Low Patrol, Swooper & Swamp/Marsh, Freshwater \\
\hline RITR & Cup & Fish, Aquatic Inverts & Water & Surface Dips, High Dives & Pelagic, Coastal \\
\hline RIBR & Cup & Fish, Aquatic Inverts & Water & High Dives, Hover \& Pick & Pelagic, Coastal \\
\hline CACA & Scrape & Insects, Seeds & Ground & Ground Gleaner, Prober & Tundra \\
\hline ERAL & Saucer & Seeds, Insects & Ground & Ground Gleaner & Grassland, Cropland \\
\hline ARGU & Saucer & Aquatic Inverts & Water & Prober, Ambusher & Swamp/Marsh \\
\hline CAOR & Cup & Insects, Seeds & Ground & Ground Gleaner & Grassland \\
\hline CALA & Cup & Insects, Seeds & Ground & Ground Gleaner & Grassland, Shrubland \\
\hline
\end{tabular}




\begin{tabular}{|c|c|c|c|c|c|c|}
\hline COMMON NAME & SCIENTIFIC NAME & $\begin{array}{l}\text { LCTA } \\
\text { CODE }\end{array}$ & $\begin{array}{l}\text { ALPHA } \\
\text { CODE }\end{array}$ & AOU\# & $\begin{array}{l}\text { NEO } \\
\text { STAT }\end{array}$ & NEST LOCATION \\
\hline Longspur, McCown's & Calcarius mccownii & CAMC & MCLO & 5390 & $\mathrm{~B}$ & Ground \\
\hline Longspur, Smith’s & Calcarius pictus & CAPI & SMLO & 5370 & $\mathrm{R}$ & Ground \\
\hline Loon, Arctic & Gavia arctica & GAAR & ARLO & 0090 & $\mathrm{R}$ & Ground \\
\hline Loon, Common & Gavia immer & GAIM & COLO & 0070 & $\mathrm{R}$ & Ground \\
\hline Loon, Pacific & Gavia pacifica & GAPA & PALO & 0100 & $\mathrm{R}$ & Ground \\
\hline Loon, Red-throated & Gavia stellata & GAST2 & RTLO & 0110 & $\mathrm{R}$ & Ground \\
\hline Loon, Yellow-billed & Gavia adamsii & GAAD & YBLO & 0080 & $\mathrm{R}$ & Ground \\
\hline Magpie, Black-billed & Pica pica & PIPI & BBMA & 4750 & $\mathrm{R}$ & Woody Upper Canopy, Shrub \\
\hline Magpie, Yellow-billed & Pica nuttalli & PINU & YBMA & 4760 & $\mathrm{R}$ & Woody Upper Canopy \\
\hline Mallard & Anas platyrhynchos & ANPL & MALL & 1320 & $\mathrm{R}$ & Ground \\
\hline Martin, Purple & Progne subis & PRSU & PUMA & 6110 & $A$ & Snag, MMS \\
\hline Meadowlark, Eastern & Sturnella magna & STMA2 & EAME & 5010 & B & Ground \\
\hline Meadowlark, Western & Sturnella neglecta & STNE1 & WEME & 5011 & $\mathrm{~B}$ & Ground \\
\hline Merganser, Common & Mergus merganser & MEME2 & COME & 1290 & $\mathrm{R}$ & Woody Upper Canopy, Ground \\
\hline Merganser, Hooded & Lophodytes cucullatus & LOCU1 & HOME & 1310 & $\mathrm{R}$ & Snag, Woody Lower Canopy \\
\hline Merganser, Red-breasted & Mergus serrator & MESE & RBME & 1300 & $\mathrm{R}$ & Ground \\
\hline Merlin & Falco columbarius & FACO & MERL & 3570 & $A$ & Woody Upper Canopy, Cliff \\
\hline Mockingbird, Northern & Mimus polyglottos & MIPO & NOMO & 7030 & $\mathrm{~B}$ & Shrub, Woody Lower Canopy \\
\hline Moorhen, Common & Gallinula chloropus & $\mathrm{GACH}$ & COMO & 2190 & $\mathrm{R}$ & Floating, Ground \\
\hline Murre, Common & Uria aalge & URAA & COMU & 0300 & $\mathrm{R}$ & Cliff \\
\hline Murre, Thick-billed & Uria lomvia & URLO & TBMU & 0310 & $\mathrm{R}$ & Cliff \\
\hline Murrelet, Ancient & Synthliboramphus antiquus & SYAN & ANMU & 0210 & $\mathrm{R}$ & Ground \\
\hline Murrelet, Kittlitz's & Brachyramphus brevirostris & BRBR & KIMU & 0240 & $\mathrm{R}$ & Ground \\
\hline Murrelet, Marbled & Brachyramphus marmoratus & BRMA & MAMU & 0230 & $\mathrm{R}$ & Ground, Woody Upper Canopy \\
\hline Murrelet, Xantus' & Synthliboramphus hypoleucus & SYHY & XAMU & 0250 & $\mathrm{R}$ & Ground \\
\hline Myna, Crested & Acridotheres cristatellus & ACCR & CRMY & 4931 & $\mathrm{R}$ & Woody Upper Canopy, MMS \\
\hline Nighthawk, Antillean & Chordeiles gundlachii & CHGU & ANNI & 4201 & $\mathrm{D}$ & Ground \\
\hline Nighthawk, Common & Chordeiles minor & $\mathrm{CHMI}$ & CONI & 4200 & $A$ & Ground \\
\hline Nighthawk, Lesser & Chordeiles acutipennis & $\mathrm{CHAC}$ & LENI & 4210 & $A$ & Ground \\
\hline Nightjar, Buff-collared & Caprimulgus ridgwayi & CARI & $\mathrm{BCNI}$ & 4161 & $\mathrm{C}$ & Ground \\
\hline Noddy, Brown & Anous stolidus & ANST2 & BRNO & 0790 & $\mathrm{R}$ & Shrub, Woody Lower Canopy \\
\hline Nutcracker, Clark's & Nucifraga columbiana & NUCO & CLNU & 4910 & $\mathrm{R}$ & Woody Upper Canopy \\
\hline Nuthatch, Brown-headed & Sitta pusilla & SIPU & $\mathrm{BHNU}$ & 7290 & $\mathrm{R}$ & Snag, Woody Lower Canopy \\
\hline Nuthatch, Pygmy & Sitta pygmaea & SIPY & PYNU & 7300 & $\mathrm{R}$ & Woody Upper Canopy, Snag \\
\hline Nuthatch, Red-breasted & Sitta canadensis & SICA1 & RBNU & 7280 & $\mathrm{R}$ & Woody Upper Canopy \\
\hline Nuthatch, White-breasted & Sitta carolinensis & SICA2 & WBNU & 7270 & $\mathrm{R}$ & Woody Upper Canopy \\
\hline Oldsquaw & Clangula hyemalis & CLHY & OLDS & 1540 & $\mathrm{R}$ & Ground \\
\hline Oriole, Altamira & Icterus gularis & ICGU & ALOR & 5031 & $\mathrm{R}$ & Woody Upper Canopy \\
\hline Oriole, Audubon's & Icterus graduacauda & ICGR & AUOR & 5030 & $\mathrm{R}$ & Woody Lower Canopy \\
\hline Oriole, Hooded & Icterus cucullatus & ICCU & HOOR & 5050 & $A$ & Woody Upper Canopy, Shrub \\
\hline Oriole, Northern & Icterus galbula & ICGA & NOOR $^{*}$ & 5070 & $A$ & Woody Upper Canopy \\
\hline Oriole, Orchard & Icterus spurius & ICSP & OROR & 5060 & $A$ & Woody Upper Canopy, Shrub \\
\hline Oriole, Scott's & Icterus parisorum & ICPA & SCOR & 5040 & $A$ & Woody Lower Canopy, Shrub \\
\hline Oriole, Spot-breasted & Icterus pectoralis & ICPE & SPOO & 5032 & $\mathrm{R}$ & Woody Upper Canopy \\
\hline Osprey & Pandion haliaetus & PAHA & OSPR & 3640 & $\mathrm{R}$ & Woody Upper Canopy, Cliff \\
\hline Ovenbird & Seiurus aurocapillus & SEAU & OVEN & 6740 & $A$ & Ground, Woody Lower Canopy \\
\hline Owl, Barred & Strix varia & STVA & BAOW & 3680 & $\mathrm{R}$ & Snag, Woody Upper Canopy \\
\hline Owl, Boreal & Aegolius funereus & AEFU & Boow & 3710 & $\mathrm{R}$ & Snag, Woody Lower Canopy \\
\hline
\end{tabular}




\begin{tabular}{|c|c|c|c|c|c|}
\hline $\begin{array}{l}\text { LCTA } \\
\text { CODE }\end{array}$ & NEST TYPE & FOOD TYPE & $\begin{array}{l}\text { FORAGING } \\
\text { SUBSTRATE }\end{array}$ & FORAGING TECHNIQUE & GE NERAL HABITAT \\
\hline CAMC & Cup & Seeds, Insects & Ground & Ground Gleaner & Grassland, Cropland \\
\hline CAPI & Cup & Insects, Seeds & Ground & Ground Gleaner & Grassland, Tundra \\
\hline GAAR & Platform & Fish, Insects & Water, Ground & Surface Dives, Ground Gleaner & Freshwater, Tundra \\
\hline GAIM & Platform & Fish, Aquatic Inverts & Water & Surface Dives & Freshwater \\
\hline GAPA & Platform & Fish, Insects & Water & Surface Dives & Tundra, Pelagic \\
\hline GAAD & Saucer & Fish, Aquatic Inverts & Water & Surface Dives & Freshwater, Tundra \\
\hline PIPI & Sphere & Insects, Carrion & Ground & Ground Gleaner & Shrubland, Riparian \\
\hline PINU & Sphere & Insects, Carrion & Ground, Foliage & Ground Gleaner, Foliage Gleaner & Forest, Riparian \\
\hline ANPL & Scrape & Seeds, Greens & Water & Dabbler & Freshwater, Swamp/Marsh \\
\hline PRSU & Cavity & Insects & Air & Aerial Foraging & Grassland, Urban \\
\hline LOCU1 & Cavity & Fish, Aquatic Inverts & Water & Surface Dives & Freshwater, Swamp/Marsh \\
\hline MESE & Scrape & Fish, Aquatic Inverts & Water & Surface Dives & Freshwater \\
\hline FACO & Platform, Cavity & Birds, Small Mammals & Air & Aerial Pursuit, Hawker & Forest, Grassland \\
\hline MIPO & Cup & Insects, Fruit & Ground, Foliage & Ground Gleaner, Foliage Gleaner & Shrubland, Urban \\
\hline $\mathrm{GACH}$ & Platform, Cup & Greens, Aquatic Inverts & Water, Ground & Surface Dips, Ground Gleaner & Swamp/Marsh, Freshwater \\
\hline URAA & None & Fish, Aquatic Inverts & Water & Surface Dives & Coastal, Pelagic \\
\hline URLO & None & Fish, Aquatic Inverts & Water & Surface Dives & Coastal, Pelagic \\
\hline SYAN & Burrow, Crevice & Aquatic Inverts, Fish & Water & Surface Dives & Coastal, Pelagic \\
\hline BRBR & None & Aquatic Inverts & Water & Surface Dives & Coastal, Pelagic \\
\hline BRMA & Saucer & Fish, Aquatic Inverts & Water & Surface Dives & Coastal, Pelagic \\
\hline ANST2 & Saucer & Fish & Water & Skimmer & Pelagic, Shoreline \\
\hline NUCO & Cup & Nuts, Seeds & Foliage, Ground & Foliage Gleaner, Ground Gleaner & Forest \\
\hline SIPU & Cavity & Insects, Seeds & Bark, Air & Bark Gleaner, Hawker & Forest \\
\hline SIPY & Cavity & Insects, Seeds & Bark, Air & Bark Gleaner, Hover Gleaner & Forest \\
\hline SICA1 & Cavity & Insects, Seeds & Bark, Air & Bark Gleaner, Hawker & Forest \\
\hline SICA2 & Cavity & Insects, Nuts & Bark & Bark Gleaner & Forest, Forest Edge \\
\hline CLHY & Scrape & Aquatic Inverts, Fish & Water & Surface Dives & Coastal, Tundra \\
\hline ICGU & Pendant & Insects, Fruit & Foliage & Foliage Gleaner & Forest, Shrubland \\
\hline ICGR & Pendant & Insects, Fruit & Foliage & Foliage Gleaner & Shrubland, Riparian \\
\hline ICCU & Pendant & Insects, Nectar & Foliage & Foliage Gleaner & Riparian, Shrubland \\
\hline ICGA & Pendant & Insects, Fruit & Foliage, Air & Foliage Gleaner, Hawker & Forest Edge, Urban \\
\hline ICSP & Pendant & Insects, Fruit & Foliage & Foliage Gleaner & Shrubland, Forest \\
\hline ICPA & Pendant & Insects, Fruit & Foliage & Foliage Gleaner & Shrubland, Riparian \\
\hline ICPE & Pendant & Fruit, Nectar & Foliage & Foliage Gleaner & Forest, Shrubland \\
\hline PAHA & Platform & Fish, Small Mammals & Water & High Dives & Freshwater \\
\hline SEAU & Oven & Insects & Ground & Ground Gleaner & Forest \\
\hline STVA & Cavity & Small Mammals, Birds & Ground & Low Patrol, Hover \& Pounce & Forest, Swamp/Marsh \\
\hline AEFU & Cavity & Small Mammals, Birds & Ground & Swooper & Forest, Swamp/Marsh \\
\hline
\end{tabular}




\begin{tabular}{|c|c|c|c|c|c|c|}
\hline COMMON NAME & SCIENTIFIC NAME & $\begin{array}{l}\text { LCTA } \\
\text { CODE }\end{array}$ & $\begin{array}{l}\text { ALPHA } \\
\text { CODE }\end{array}$ & AOU\# & $\begin{array}{l}\text { NEO } \\
\text { STAT }\end{array}$ & NEST LOCATION \\
\hline Owl, Burrowing & Athene cunicularia & ATCU & BUOW & 3780 & $\mathrm{~A}$ & Ground \\
\hline Owl, Common Barn & Tyto alba & TYAL & COBO & 3650 & $\mathrm{R}$ & Snag, MMS \\
\hline Owl, Eastern Screech & Otus asio & OTAS & EASO & 3730 & $\mathrm{R}$ & Snag, Woody Upper Canopy \\
\hline Owl, Elf & Micrathene whitneyi & MIWH & ELOW & 3810 & $\mathrm{C}$ & Cactus, Woody Upper Canopy \\
\hline Owl, Ferruginous Pygmy & Glaucidium brasilianum & GLBR & FEPO & 3800 & $\mathrm{R}$ & Snag, Woody Lower Canopy \\
\hline Owl, Flammulated & Otus flammeolus & OTFL & FLOW & 3740 & A & Snag \\
\hline Owl, Great Gray & Strix nebulosa & STNE & GGOW & 3700 & $\mathrm{R}$ & Woody Upper Canopy \\
\hline Owl, Great Horned & Bubo virginianus & BUVI & GHOW & 3750 & $\mathrm{R}$ & Woody Upper Canopy, Cliff \\
\hline Owl, Long-eared & Asio otus & ASOT & LEOW & 3660 & B & Woody Upper Canopy, Ground \\
\hline Owl, Northern Hawk & Surnia ulula & SUUL & $\mathrm{NOHO}$ & 3770 & $\mathrm{R}$ & Snag, Woody Upper Canopy \\
\hline Owl, Northern Pygmy & Glaucidium gnoma & GLGN & NOPO & 3790 & $\mathrm{R}$ & Snag, Woody Lower Canopy \\
\hline Owl, Northern Saw-whet & Aegolius acadicus & AEAC & NSWO & 3720 & $\mathrm{R}$ & Snag, Woody Upper Canopy \\
\hline Owl, Short-eared & Asio flammeus & ASFL & SEOW & 3670 & B & Ground \\
\hline Owl, Snowy & Nyctea scandiaca & NYSC & SNOW & 3760 & $\mathrm{R}$ & Ground \\
\hline Owl, Spotted & Strix occidentalis & STOC1 & SPOW & 3690 & $\mathrm{R}$ & Woody Upper Canopy, Cliff \\
\hline Owl, Western Screech & Otus kennicotti & OTKE & WESO & 3732 & $\mathrm{R}$ & Snag, Woody Upper Canopy \\
\hline Owl, Whiskered Screech & Otus trichopsis & OTTR & WHSO & 3731 & $\mathrm{R}$ & Snag \\
\hline Oystercatcher, American & Haematopus palliatus & HAPA & AMOY & 2860 & $\mathrm{R}$ & Ground \\
\hline Oystercatcher, Black & Haematopus bachmani & HABA & BLOY & 2870 & $\mathrm{R}$ & Ground \\
\hline Partridge, Gray & Perdix perdix & PEPE & GRPA* & 2881 & $\mathrm{R}$ & Ground \\
\hline Parula, Northern & Parula americana & PAAM & NOPA & 6480 & A & Woody Upper Canopy \\
\hline Parula, Tropical & Parula pitiayumi & PAPI & TRPA & 6490 & $\mathrm{R}$ & Woody Upper Canopy \\
\hline Pauraque, Common & Nyctidromus albicollis & NYAL & COPA & 4190 & $\mathrm{R}$ & Ground \\
\hline Pelican, American White & Pelecanus erythrorhynchos & PEER & AWPE & 1250 & $\mathrm{R}$ & Ground \\
\hline Pelican, Brown & Pelecanus occidentalis & PEOC & BRPE & 1260 & $\mathrm{R}$ & Woody Upper Canopy, Cliff \\
\hline Petrel, Ashy Storm & Oceanodroma homochroa & $\mathrm{OCHO}$ & ASSP & 1080 & $\mathrm{R}$ & Ground \\
\hline Petrel, Black Storm & Oceanodroma melania & OCME & BLSP & 1070 & $\mathrm{R}$ & Ground, Cliff \\
\hline Petrel, British Storm & Hydrobates pelagicus & HYPE & $\mathrm{BHSP}^{*}$ & 1040 & $\mathrm{R}$ & Ground, Cliff \\
\hline Petrel, Fork-tailed Storm & Oceanodroma furcata & OCFU & FTSP & 1050 & $\mathrm{R}$ & Cliff \\
\hline Petrel, Leach's Storm & Oceanodroma leucorhoa & OCLE & LHSP & 1060 & $\mathrm{R}$ & Ground, Bank \\
\hline Petrel, Least Storm & Oceanodroma microsoma & OCMI & LTSP & 1030 & $\mathrm{R}$ & Ground, Cliff \\
\hline Petrel, Wilson's Storm & Oceanites oceanicus & OCOC & WISP & 1090 & $\mathrm{R}$ & Ground, Cliff \\
\hline Pewee, Eastern Wood & Contopus virens & COVI1 & EAWP & 4610 & $A$ & Woody Upper Canopy \\
\hline Pewee, Greater & Contopus pertinax & COPE & GRPE & 4600 & C & Woody Upper Canopy \\
\hline Pewee, Western Wood & Contopus sordidulus & coso & WEWP & 4620 & A & Woody Upper Canopy \\
\hline Phainopepla & Phainopepla nitens & PHNI & PHAI & 6200 & A & Woody Upper Canopy, Shrub \\
\hline Phalarope, Red & Phalaropus fulicarius & PHFU & REPH & 2220 & $\mathrm{R}$ & Ground \\
\hline Phalarope, Red-necked & Phalaropus lobatus & PHLO & $\mathrm{RNPH}$ & 2230 & $\mathrm{R}$ & Ground \\
\hline Phalarope, Wilson's & Phalaropus tricolor & PHTR & WIPH & 2240 & $\mathrm{R}$ & Ground \\
\hline Pheasant, Ring-necked & Phasianus colchicus & $\mathrm{PHCO}$ & RNPS* & 3091 & $\mathrm{R}$ & Ground \\
\hline Phoebe, Black & Sayornis nigricans & SANI & BLPH & 4580 & $\mathrm{R}$ & Cliff, MMS \\
\hline Phoebe, Eastern & Sayornis phoebe & SAPH & EAPH & 4560 & B & MMS, Cliff \\
\hline Phoebe, Say's & Sayornis saya & SASA & SAPH & 4570 & $\mathrm{~B}$ & Cliff, MMS \\
\hline Pigeon, Band-tailed & Columba fasciata & COFA & BTPI & 3120 & A & Woody Upper Canopy \\
\hline Pigeon, Red-billed & Columba flavirostris & COFL1 & RBPI & 3130 & $\mathrm{R}$ & Woody Upper Canopy \\
\hline Pigeon, White-crowned & Columba leucocephala & COLE & WCPI & 3140 & $\mathrm{D}$ & Woody Lower Canopy \\
\hline Pintail, Northern & Anas acuta & ANAC & NOPI & 1430 & $\mathrm{R}$ & Ground \\
\hline Pipit, Red-throated & Anthus cervinus & ANCE & RTPI & 6990 & $\mathrm{R}$ & Ground \\
\hline
\end{tabular}




\begin{tabular}{|c|c|c|c|c|c|}
\hline $\begin{array}{l}\text { LCTA } \\
\text { CODE }\end{array}$ & NEST TYPE & FOOD TYPE & $\begin{array}{l}\text { FORAGING } \\
\text { SUBSTRATE }\end{array}$ & FORAGING TECHNIQUE & GE NERAL HABITAT \\
\hline ATCU & Burrow & Insects, Small Mammals & Ground & Swooper, Hover \& Pounce & Grassland, Urban \\
\hline TYAL & Cavity & Small Mammals, Birds & Ground & Low Patrol, Swooper & Grassland, Urban \\
\hline OTAS & Cavity & Insects, Small Verts & Ground & Swooper & Forest, Riparian \\
\hline MIWH & Cavity & Insects & Ground & Low Patrol, Hover \& Pounce & Desert, Shrubland \\
\hline GLBR & Cavity & Insects, Small Verts & Ground & Swooper & Desert, Shrubland \\
\hline STNE & Abandoned & Small Mammals, Birds & Ground & Hover \& Pounce, Swooper & Forest, Swamp/Marsh \\
\hline BUVI & Abandoned & Small Mammals, Birds & Ground & Swooper & Forest, Swamp/Marsh \\
\hline ASOT & Abandoned & Small Mammals, Birds & Ground & Low Patrol & Forest, Urban \\
\hline SUUL & Cavity, Platform & Small Mammals, Birds & Ground & Swooper, Hover \& Pounce & Forest, Swamp/Marsh \\
\hline GLGN & Cavity & Small Mammals, Insects & Ground & Swooper & Forest \\
\hline STOC1 & Cavity, Platform & Small Mammals, Birds & Ground & Swooper & Forest \\
\hline OTKE & Cavity & Small Mammals, Insects & Ground & Swooper & Riparian, Shrubland \\
\hline OTTR & Cavity & Insects & Ground & Swooper & Forest \\
\hline HAPA & Scrape & Aquatic Inverts, Fish & Water & Prober & Coastal, Pelagic \\
\hline HABA & Scrape & Aquatic Inverts, Fish & Water & Prober & Coastal, Pelagic \\
\hline PEPE & Scrape & Seeds, Insects & Ground & Ground Gleaner & Grassland \\
\hline PAAM & Pendant & Insects & Foliage, Air & Foliage Gleaner, Hover Gleaner & Forest \\
\hline PAPI & Pendant & Insects & Foliage, Air & Foliage Gleaner, Hawker & Forest \\
\hline NYAL & None & Insects & Air & Aerial Foraging, Hawker & Forest, Forest Edge \\
\hline PEER & Scrape & Fish, Aquatic Inverts & Water & Surface Dips & Coastal, Pelagic \\
\hline OCMI & Burrow, Crevice & Greens, Aquatic Inverts & Water & Low Patrol & Coastal, Pelagic \\
\hline OCOC & Burrow, Crevice & Greens, Fish & Water & Scavenger, Low Patrol & Coastal, Pelagic \\
\hline COVI1 & Cup & Insects, Fruit & Air & Hawker, Hover Gleaner & Forest, Forest Edge \\
\hline COPE & Cup & Insects & Air & Hawker & Forest \\
\hline coso & Cup & Insects, Fruit & Air & Hawker, Hover Gleaner & Forest, Forest Edge \\
\hline PHNI & Cup & Fruit, Insects & Foliage, Air & Foliage Gleaner, Hawker & Shrubland, Riparian \\
\hline PHFU & Scrape & Aquatic Inverts, Insects & Water & Surface Dips, Prober & Freshwater, Tundra \\
\hline PHLO & Scrape & Aquatic Inverts, Seeds & Water & Surface Dips, Prober & Freshwater \\
\hline PHTR & Scrape & Aquatic Inverts, Seeds & Water & Surface Dips, Prober & Swamp/Marsh \\
\hline $\mathrm{PHCO}$ & Scrape & Insects, Seeds & Ground & Ground Gleaner & Grassland, Cropland \\
\hline SANI & Cup & Insects, Fish & Air & Hawker, Hover Gleaner & Riparian, Urban \\
\hline SAPH & Cup & Insects, Fish & Air & Hawker & Riparian, Urban \\
\hline SASA & Cup & Insects, Fruit & Air & Hawker, Hover Gleaner & Grassland, Forest \\
\hline COFA & Platform & Nuts, Fruit & Foliage, Ground & Foliage Gleaner, Ground Gleaner & Forest \\
\hline COFL1 & Platform & Seeds, Nuts & Foliage, Ground & Foliage Gleaner, Ground Gleaner & Forest, Riparian \\
\hline COLE & Platform & Fruit & Foliage & Foliage Gleaner & Swamp/Marsh \\
\hline ANAC & Scrape & Seeds, Greens & Water, Ground & Dabbler, Ground Gleaner & Swamp/Marsh, Freshwater \\
\hline ANCE & Cup & Insects, Seeds & Ground & Ground Gleaner & Tundra, Rocky Slope \\
\hline
\end{tabular}




\begin{tabular}{|c|c|c|c|c|c|c|}
\hline COMMON NAME & SCIENTIFIC NAME & $\begin{array}{l}\text { LCTA } \\
\text { CODE }\end{array}$ & $\begin{array}{l}\text { ALPHA } \\
\text { CODE }\end{array}$ & AOU\# & $\begin{array}{l}\text { NEO } \\
\text { STAT }\end{array}$ & NEST LOCATION \\
\hline Pipit, Sprague’s & Anthus spragueii & ANSP1 & SPPI & 7000 & $B$ & Ground \\
\hline Pipit, Water & Anthus spinoletta & ANSP & WAPI & 6970 & $\mathrm{~B}$ & Ground \\
\hline Plover, Black-bellied & Pluvialis squatarola & PLSQ & BBPL & 2700 & $\mathrm{R}$ & Ground \\
\hline Plover, Common Ringed & Charadrius hiaticula & $\mathrm{CHHI}$ & CRPL & 2750 & $\mathrm{R}$ & Ground \\
\hline Plover, Lesser Golden & Pluvialis dominica & PLDO1 & LEGP & 2720 & $\mathrm{R}$ & Ground \\
\hline Plover, Mongolian & Charadrius mongolus & $\mathrm{CHMO}$ & MOPL & 2790 & $\mathrm{R}$ & Ground \\
\hline Plover, Mountain & Charadrius montanus & CHMO1 & MOUP & 2810 & A & Ground \\
\hline Plover, Piping & Charadrius melodus & CHME & PIPL & 2770 & $\mathrm{R}$ & Ground \\
\hline Plover, Semipalmated & Charadrius semipalmatus & CHSE & SEPL & 2740 & $\mathrm{R}$ & Ground \\
\hline Plover, Snowy & Charadrius alexandrinus & $\mathrm{CHAL}$ & SNPL & 2780 & $\mathrm{R}$ & Ground \\
\hline Plover, Wilson's & Charadrius wilsonia & $\mathrm{CHWI}$ & WIPL & 2800 & $\mathrm{R}$ & Ground \\
\hline Poor-will, Common & Phalaenoptilus nuttallii & PHNU & COPW & 4180 & B & Ground \\
\hline Prairie-chicken, Greater & Tympanuchus cupido & TYCU & $\mathrm{GRPC}^{*}$ & 3050 & $\mathrm{R}$ & Ground \\
\hline Prairie-chicken, Lesser & Tympanuchus pallidicinctus & TYPA & LEPC* & 3070 & $\mathrm{R}$ & Ground \\
\hline Ptarmigan, Rock & Lagopus mutus & LAMU & $\mathrm{ROPT}^{*}$ & 3020 & $\mathrm{R}$ & Ground \\
\hline Ptarmigan, White-tailed & Lagopus leucurus & LALE & WTPT* & 3040 & $\mathrm{R}$ & Ground \\
\hline Ptarmigan, Willow & Lagopus lagopus & LALA & WIPT* $^{*}$ & 3010 & $\mathrm{R}$ & Ground \\
\hline Puffin, Atlantic & Fratercula arctica & FRAR & ATPU & 0130 & $\mathrm{R}$ & Ground, Cliff \\
\hline Puffin, Horned & Fratercula corniculata & FRCO & HOPU & 0140 & $\mathrm{R}$ & Ground, Cliff \\
\hline Puffin, Tufted & Fratercula cirrhata & $\mathrm{FRCl}$ & TUPU & 0120 & $\mathrm{R}$ & Ground, Cliff \\
\hline Pyrrhuloxia & Cardinalis sinuatus & CASI & PYRR & 5940 & $\mathrm{R}$ & Shrub, Woody Lower Canopy \\
\hline Quail, California & Callipepla californica & CACA1 & CAQU* & 2940 & $\mathrm{R}$ & Ground \\
\hline Quail, Gambel's & Callipepla gambelii & CAGA1 & GAQU* & 2950 & $\mathrm{R}$ & Ground \\
\hline Quail, Montezuma & Cyrtonyx montezumae & CYMO1 & MZQU* & 2960 & $\mathrm{R}$ & Ground \\
\hline Quail, Mountain & Oreortyx pictus & ORPI & MNQU* & 2920 & $\mathrm{R}$ & Ground \\
\hline Quail, Scaled & Callipepla squamata & CASQ & SCQU* & 2930 & $\mathrm{R}$ & Ground \\
\hline Rail, Black & Laterallus jamaicensis & LAJA & BLRA & 2160 & $\mathrm{R}$ & Ground \\
\hline Rail, Clapper & Rallus longirostris & RALO & CLRA & 2110 & $\mathrm{R}$ & Ground \\
\hline Rail, King & Rallus elegans & RAEL & KIRA & 2080 & $\mathrm{R}$ & Ground \\
\hline Rail, Virginia & Rallus limicola & RALI & VIRA & 2120 & $\mathrm{R}$ & Ground \\
\hline Rail, Yellow & Coturnicops noveboracensis & CONO & YERA & 2150 & $\mathrm{R}$ & Ground \\
\hline Raven, Chihuahuan & Corvus cryptoleucus & COCR1 & CHRA & 4870 & $\mathrm{R}$ & Woody Upper Canopy, Shrub \\
\hline Raven, Common & Corvus corax & $\mathrm{COCO} 2$ & CORA & 4860 & $\mathrm{R}$ & Cliff, Woody Upper Canopy \\
\hline Razorbill & Alca torda & ALTO & RAZO & 0320 & $\mathrm{R}$ & Cliff \\
\hline Redhead & Aythya americana & AYAM & REDH & 1460 & $\mathrm{R}$ & Floating \\
\hline Redpoll, Common & Carduelis flammea & CAFL & CORE & 5280 & $\mathrm{R}$ & Shrub, Ground \\
\hline Redpoll, Hoary & Carduelis hornemanni & $\mathrm{CAHO}$ & HORE & 5270 & $\mathrm{R}$ & Shrub, Ground \\
\hline Redstart, American & Setophaga ruticilla & SERU1 & AMRE & 6870 & A & Woody Lower Canopy, Shrub \\
\hline Redstart, Painted & Myioborus pictus & MYPI & PARE & 6880 & C & Ground, Bank \\
\hline Roadrunner, Greater & Geococcyx californianus & GECA1 & GRRO & 3850 & $\mathrm{R}$ & Woody Lower Canopy, Shrub \\
\hline Robin, American & Turdus migratorius & TUMI & AMRO & 7610 & B & Woody Lower Canopy \\
\hline Sanderling & Calidris alba & CAAL & SAND & 2480 & $\mathrm{R}$ & Ground \\
\hline Sandpiper, Bairds & Calidris bairdii & CABA & BASA & 2410 & $\mathrm{R}$ & Ground \\
\hline Sandpiper, Buff-breasted & Tryngites subruficollis & TRSU & BBSA & 2620 & $\mathrm{R}$ & Ground \\
\hline Sandpiper, Curlew & Calidris ferruginea & CAFE & CUSA & 2440 & $\mathrm{R}$ & Ground \\
\hline Sandpiper, Least & Calidris minutilla & CAMI1 & LESA & 2420 & $\mathrm{R}$ & Ground \\
\hline Sandpiper, Pectoral & Calidris melanotos & CAME1 & PESA & 2390 & $\mathrm{R}$ & Ground \\
\hline Sandpiper, Purple & Calidris maritima & CAMA & PUSA & 2350 & $\mathrm{R}$ & Ground \\
\hline
\end{tabular}




\begin{tabular}{|c|c|c|c|c|c|}
\hline $\begin{array}{l}\text { LCTA } \\
\text { CODE }\end{array}$ & NEST TYPE & FOOD TYPE & $\begin{array}{l}\text { FORAGING } \\
\text { SUBSTRATE }\end{array}$ & FORAGING TECHNIQUE & GENERAL HABITAT \\
\hline ANSP1 & Cup & Insects, Seeds & Ground & Ground Gleaner & Grassland \\
\hline ANSP & Cup & Insects, Aquatic Inverts & Ground, Air & Ground Gleaner, Hawker & Shoreline, Tundra \\
\hline PLSQ & Scrape & Insects & Ground, Water & Ground Gleaner, Prober & Coastal, Tundra \\
\hline $\mathrm{CHHI}$ & Scrape & Insects & Ground, Water & Ground Gleaner, Prober & Coastal, Tundra \\
\hline PLDO1 & Scrape & Insects & Ground, Water & Ground Gleaner, Prober & Coastal, Tundra \\
\hline $\mathrm{CHMO1}$ & Scrape & Insects & Ground, Water & Ground Gleaner & Grassland \\
\hline CHME & Scrape & Aquatic Inverts, Insects & Ground, Water & Ground Gleaner, Prober & Coastal, Freshwater \\
\hline CHSE & Scrape & Aquatic Inverts, Insects & Ground, Water & Ground Gleaner & Coastal, Tundra \\
\hline CHAL & Scrape & Insects, Aquatic Inverts & Ground, Water & Ground Gleaner, Prober & Coastal, Freshwater \\
\hline $\mathrm{CHWI}$ & Scrape & Aquatic Inverts, Insects & Water & Prober & Coastal \\
\hline LAMU & Scrape & Greens, Fruit & Ground & Ground Gleaner & Rocky Slope, Tundra \\
\hline LALE & Scrape & Greens, Insects & Ground & Ground Gleaner & Tundra \\
\hline LALA & Scrape & Greens, Seeds & Foliage, Ground & Foliage Gleaner, Ground Gleaner & Shrubland, Tundra \\
\hline FRAR & Burrow, Crevice & Fish, Aquatic Inverts & Water & Surface Dives & Coastal, Pelagic \\
\hline FRCO & Burrow, Crevice & Fish, Aquatic Inverts & Water & Surface Dives & Coastal, Pelagic \\
\hline $\mathrm{FRCl}$ & Burrow, Crevice & Fish, Aquatic Inverts & Water & Surface Dives & Coastal, Pelagic \\
\hline CASI & Cup & Seeds, Insects & Ground & Ground Gleaner & Shrubland \\
\hline CACA1 & Scrape & Seeds, Nuts & Ground & Ground Gleaner & Shrubland, Cropland \\
\hline CAGA1 & Scrape & Seeds, Greens & Ground & Ground Gleaner, Foliage Browser & Shrubland, Riparian \\
\hline CYMO1 & Scrape & Greens, Insects & Ground & Digging, Ground Gleaner & Forest \\
\hline CONO & Saucer & Aquatic Inverts, Insects & Water, Ground & Prober, Ground Gleaner & Swamp/Marsh, Freshwater \\
\hline COCR1 & Cup & Insects, Carrion & Ground & Scavenger, Ground Gleaner & Grassland, Shrubland \\
\hline $\mathrm{COCO} 2$ & Cup & Carrion, Small Verts & Ground & Scavenger, Ground Gleaner & Forest, Grassland \\
\hline ALTO & Crevice & Fish, Aquatic Inverts & Water & Surface Dives & Coastal, Pelagic \\
\hline AYAM & Cup & Greens, Aquatic Inverts & Water & Surface Dives & Swamp/Marsh, Freshwater \\
\hline CAFL & Cup, Crevice & Seeds, Insects & Foliage, Ground & Foliage Gleaner, Ground Gleaner & Forest, Tundra \\
\hline $\mathrm{CAHO}$ & Cup, Crevice & Seeds, Insects & Foliage, Ground & Foliage Gleaner, Ground Gleaner & Shrubland, Tundra \\
\hline SERU1 & Cup & Insects, Seeds & Air, Foliage & Hover Gleaner, Foliage Gleaner & Forest, Forest Edge \\
\hline MYPI & Cup & Insects & Foliage, Bark & Foliage Gleaner, Bark Gleaner & Forest, Riparian \\
\hline GECA1 & Platform & Insects, Small Mammals & Ground & Ground Gleaner & Shrubland, Desert \\
\hline TUMI & Cup & Insects & Ground, Foliage & Ground Gleaner, Foliage Gleaner & Forest, Urban \\
\hline CAAL & Scrape & Insects, Greens & Water, Ground & Prober, Ground Gleaner & Coastal, Tundra \\
\hline CABA & Scrape & Insects & Ground & Ground Gleaner & Shoreline, Tundra \\
\hline TRSU & Scrape & Insects, Seeds & Ground & Ground Gleaner & Shoreline, Tundra \\
\hline CAFE & Burrow, Scrape & Insects, Aquatic Inverts & Water & Prober & Coastal \\
\hline CAMI1 & Scrape & Insects, Aquatic Inverts & Ground, Water & Ground Gleaner, Prober & Coastal, Swamp/Marsh \\
\hline CAME1 & Scrape & Insects & Water, Ground & Prober, Ground Gleaner & Coastal, Tundra \\
\hline CAMA & Scrape & Insects, Aquatic Inverts & Ground, Water & Ground Gleaner, Prober & Coastal, Tundra \\
\hline
\end{tabular}




\begin{tabular}{|c|c|c|c|c|c|c|}
\hline COMMON NAME & SCIENTIFIC NAME & $\begin{array}{l}\text { LCTA } \\
\text { CODE }\end{array}$ & $\begin{array}{l}\text { ALPHA } \\
\text { CODE }\end{array}$ & AOU\# & $\begin{array}{l}\text { NEO } \\
\text { STAT }\end{array}$ & NEST LOCATION \\
\hline Sandpiper, Rock & Calidris ptilocnemis & CAPT & ROSA & 2360 & $\mathrm{R}$ & Ground \\
\hline Sandpiper, Semipalmated & Calidris pusilla & CAPU & SESA & 2460 & $\mathrm{R}$ & Ground \\
\hline Sandpiper, Solitary & Tringa solitaria & TRSO & SOSA & 2560 & $\mathrm{R}$ & Woody Upper Canopy \\
\hline Sandpiper, Spotted & Actitis macularia & ACMA & SPSA & 2630 & $\mathrm{R}$ & Ground \\
\hline Sandpiper, Stilt & Calidris himantopus & CAHI & STSA & 2330 & $\mathrm{R}$ & Ground \\
\hline Sandpiper, Upland & Bartramia longicauda & BALO & UPSA & 2610 & $A$ & Ground \\
\hline Sandpiper, Western & Calidris mauri & CAMA1 & WESA & 2470 & $\mathrm{R}$ & Ground \\
\hline Sandpiper, White-rumped & Calidris fuscicollis & CAFU & WRSA & 2400 & $\mathrm{R}$ & Ground \\
\hline Sandpiper, Wood & Tringa glareola & TRGL & WOSA* & 2571 & $\mathrm{R}$ & Ground, Woody Lower Canopy \\
\hline Sapsucker, Red-breasted & Sphyrapicus ruber & SPRU & RBSA & 4030 & B & Woody Upper Canopy, Snag \\
\hline Sapsucker, Red-naped & Sphyrapicus nuehalis & SPNU & RNSA & 4021 & $\mathrm{~B}$ & Woody Lower Canopy \\
\hline Sapsucker, Williamson's & Sphyrapicus thyroideus & SPTH & WISA & 4040 & B & Woody Upper Canopy \\
\hline Sapsucker, Yellow-bellied & Sphyrapicus varius & SPVA1 & YBSA & 4020 & $\mathrm{~B}$ & Woody Upper Canopy \\
\hline Scaup, Greater & Aythya marila & AYMA & GRSC & 1480 & $\mathrm{R}$ & Ground, Floating \\
\hline Scaup, Lesser & Aythya affinis & AYAF & LESC & 1490 & $\mathrm{R}$ & Ground \\
\hline Scoter, Black & Melanitta nigra & MENI & BLSC & 1630 & $\mathrm{R}$ & Ground \\
\hline Scoter, Surf & Melanitta perspicillata & MEPE & SUSC & 1660 & $\mathrm{R}$ & Ground \\
\hline Scoter, White-winged & Melanitta fusca & MEFU & WWSC & 1650 & $\mathrm{R}$ & Ground \\
\hline Shearwater, Audubon's & Puffinus Iherminieri & PULH & $\mathrm{AUSH}$ & 0920 & $\mathrm{R}$ & Ground, Cliff \\
\hline Shearwater, Black-vented & Puffinus opisthomelas & PUOP & BVSH & 0930 & $\mathrm{R}$ & Ground, Cliff \\
\hline Shearwater, Buller's & Puffinus bulleri & PUBU & $\mathrm{BUSH}$ & 0962 & $\mathrm{R}$ & Ground \\
\hline Shearwater, Cory's & Calonectris diomedea & CADI & $\mathrm{COSH}$ & 0880 & $\mathrm{R}$ & Ground, Cliff \\
\hline Shearwater, Greater & Puffinus gravis & PUGR & GRSH & 0890 & $\mathrm{R}$ & Ground \\
\hline Shearwater, Manx & Puffinus puffinus & PUPU & MASH & 0900 & $\mathrm{R}$ & Cliff \\
\hline Shearwater, Pink-footed & Puffinus creatopus & PUCR & PFSH & 0910 & $\mathrm{R}$ & Ground \\
\hline Shearwater, Short-tailed & Puffinus tenuirostris & PUTE & SHOS & 0960 & $\mathrm{R}$ & Ground \\
\hline Shearwater, Sooty & Puffinus griseus & PUGR1 & $\mathrm{SOSH}$ & 0950 & $\mathrm{R}$ & Ground \\
\hline Shoveler, Northern & Anas clypeata & ANCL & $\mathrm{NSHO}$ & 1420 & $\mathrm{R}$ & Ground \\
\hline Shrike, Loggerhead & Lanius Iudovicianus & LALU & LOSH & 6220 & B & Woody Lower Canopy, Shrub \\
\hline Shrike, Northern & Lanius excubitor & LAEX & NSHR & 6210 & $\mathrm{R}$ & Woody Lower Canopy \\
\hline Siskin, Pine & Carduelis pinus & CAPI1 & PISI & 5330 & B & Woody Upper Canopy \\
\hline Skimmer, Black & Rynchops niger & RYNI & BLSK & 0800 & $\mathrm{R}$ & Ground \\
\hline Skua, Great & Catharacta skua & CASK & GRSK & 0350 & $\mathrm{R}$ & Ground, Cliff \\
\hline Skua, South Polar & Catharacta maccormicki & CAMA3 & SPSK & 0352 & $\mathrm{R}$ & Ground, Cliff \\
\hline Skylark, Eurasian & Alauda arvensis & ALAR & EUSK & 4730 & $\mathrm{R}$ & Ground \\
\hline Snipe, Common & Gallinago gallinago & GAGA & $\cos N$ & 2300 & $\mathrm{R}$ & Ground \\
\hline Solitaire, Townsend's & Myadestes townsendi & MYTO & TOSO & 7540 & B & Ground, Snag \\
\hline Sora & Porzana carolina & POCA1 & SORA & 2140 & $\mathrm{R}$ & Floating, Ground \\
\hline Sparrow, American Tree & Spizella arborea & SPAR2 & ATSP & 5590 & $\mathrm{R}$ & Ground, Shrub \\
\hline Sparrow, Bachman's & Aimophila aestivalis & AIAE & BACS & 5750 & $\mathrm{R}$ & Ground \\
\hline Sparrow, Baird's & Ammodramus bairdii & AMBA & BAIS & 5450 & A & Ground \\
\hline Sparrow, Black-chinned & Spizella atrogularis & SPAT & BCSP & 5650 & $A$ & Shrub, Woody Lower Canopy \\
\hline Sparrow, Black-throated & Amphispiza bilineata & AMBI & BTSP & 5730 & $\mathrm{~B}$ & Shrub, Cactus \\
\hline Sparrow, Botteri's & Aimophila botterii & AIBO & BOSP & 5760 & $\mathrm{C}$ & Ground \\
\hline Sparrow, Brewer's & Spizella breweri & SPBR1 & BRSP & 5620 & $A$ & Shrub, Woody Lower Canopy \\
\hline Sparrow, Cassin's & Aimophila cassinii & AICA1 & CASP & 5780 & $\mathrm{~B}$ & Ground, Shrub \\
\hline Sparrow, Chipping & Spizella passerina & SPPA2 & CHSP & 5600 & $A$ & Woody Lower Canopy \\
\hline Sparrow, Clay-colored & Spizella pallida & SPPA1 & CCSP & 5610 & $A$ & Shrub, Ground \\
\hline
\end{tabular}




\begin{tabular}{|c|c|c|c|c|c|}
\hline $\begin{array}{l}\text { LCTA } \\
\text { CODE }\end{array}$ & NEST TYPE & FOOD TYPE & $\begin{array}{l}\text { FORAGING } \\
\text { SUBSTRATE }\end{array}$ & FORAGING TECHNIQUE & GE NE RAL HABITAT \\
\hline CAPT & Scrape & Insects, Aquatic Inverts & Water & Prober & Coastal \\
\hline TRSO & Cup & Insects, Aquatic Inverts & Water, Ground & Prober, Ground Gleaner & Swamp/Marsh, Freshwater \\
\hline ACMA & Scrape & Insects, Aquatic Inverts & Ground & Ground Gleaner & Shoreline \\
\hline $\mathrm{CAHI}$ & Scrape & Aquatic Inverts, Greens & Water & Prober & Tundra, Swamp/Marsh \\
\hline CAMA1 & Scrape & Insects, Aquatic Inverts & Ground, Water & Ground Gleaner, Prober & Coastal \\
\hline CAFU & Scrape & Insects, Seeds & Ground, Water & Ground Gleaner, Prober & Tundra, Coastal \\
\hline TRGL & Saucer & Insects & Water, Ground & Prober, Ground Gleaner & Coastal, Tundra \\
\hline SPRU & Cavity & Insects, Tree Sap & Bark, Air & Bark Gleaner, Hawker & Forest \\
\hline SPNU & Cavity & Insects, Tree Sap & Bark, Air & Bark Gleaner, Hawker & Forest, Riparian \\
\hline AYAF & Scrape & Aquatic Inverts, Greens & Water & Surface Dives & Freshwater, Swamp/Marsh \\
\hline MENI & Scrape & Aquatic Inverts, Greens & Water & Surface Dives & Freshwater, Swamp/Marsh \\
\hline MEPE & Scrape & Aquatic Inverts, Greens & Water & Surface Dives & Freshwater, Swamp/Marsh \\
\hline MEFU & Scrape & Aquatic Inverts, Greens & Water & Surface Dives & Freshwater \\
\hline PULH & Burrow, Crevice & Fish, Aquatic Inverts & Water & Scavenger, Low Patrol & Coastal, Pelagic \\
\hline PUOP & Burrow & Fish, Aquatic Inverts & Water & Scavenger, Low Patrol & Coastal, Pelagic \\
\hline PUBU & Burrow & Fish, Aquatic Inverts & Water & Scavenger, Low Patrol & Coastal, Pelagic \\
\hline CADI & Burrow, Crevice & Fish, Aquatic Inverts & Water & Scavenger, Low Patrol & Coastal, Pelagic \\
\hline PUGR & Burrow & Fish, Aquatic Inverts & Water & Scavenger, Low Patrol & Coastal, Pelagic \\
\hline PUPU & Burrow & Fish, Aquatic Inverts & Water & Scavenger, Low Patrol & Coastal, Pelagic \\
\hline CAPI1 & Saucer & Seeds, Insects & Foliage, Ground & Foliage Gleaner, Ground Gleaner & Forest, Urban \\
\hline RYNI & Scrape & Fish, Aquatic Inverts & Water & Skimmer & Coastal, Pelagic \\
\hline CASK & Scrape & Fish, Birds & Water, Air & Scavenger, Piracy & Coastal, Pelagic \\
\hline САMA3 & Scrape & Fish, Birds & Water, Air & Scavenger, Piracy & Coastal, Pelagic \\
\hline ALAR & Saucer & Seeds, Insects & Ground, Foliage & Ground Gleaner, Foliage Gleaner & Grassland \\
\hline GAGA & Scrape & Insects & Water & Prober & Swamp/Marsh \\
\hline MYTO & Cup, Cavity & Insects, Fruit & Air, Foliage & Hawker, Foliage Gleaner & Forest \\
\hline POCA1 & Saucer & Seeds, Insects & Ground, Water & Ground Gleaner, Prober & Swamp/Marsh \\
\hline SPAR2 & Cup & Insects, Seeds & Ground, Foliage & Ground Gleaner, Foliage Gleaner & Grassland, Shrubland \\
\hline AIAE & Cup & Seeds, Insects & Ground & Ground Gleaner & Forest, Shrubland \\
\hline AMBA & Cup & Insects, Seeds & Ground & Ground Gleaner & Grassland \\
\hline SPAT & Cup & Insects, Seeds & Ground, Foliage & Ground Gleaner, Foliage Gleaner & Shrubland \\
\hline AMBI & Cup & Insects, Seeds & Ground, Foliage & Ground Gleaner, Foliage Gleaner & Shrubland \\
\hline AIBO & Cup & Insects, Seeds & Ground & Ground Gleaner & Grassland, Shrubland \\
\hline SPBR1 & Cup & Insects, Seeds & Ground & Ground Gleaner & Shrubland \\
\hline AICA1 & Cup & Insects, Seeds & Ground & Ground Gleaner & Grassland, Shrubland \\
\hline SPPA2 & Cup & Insects, Seeds & Ground, Foliage & Ground Gleaner, Foliage Gleaner & Forest, Forest Edge \\
\hline SPPA1 & Cup & Insects, Seeds & Ground & Ground Gleaner & Shrubland, Forest Edge \\
\hline
\end{tabular}




\begin{tabular}{|c|c|c|c|c|c|c|}
\hline COMMON NAME & SCIENTIFIC NAME & $\begin{array}{l}\text { LCTA } \\
\text { CODE }\end{array}$ & $\begin{array}{l}\text { ALPHA } \\
\text { CODE }\end{array}$ & AOU\# & $\begin{array}{l}\text { NEO } \\
\text { STAT }\end{array}$ & NEST LOCATION \\
\hline Sparrow, Eurasian Tree & Passer montanus & PAMO1 & ETSP & 6883 & $\mathrm{R}$ & Woody Upper Canopy \\
\hline Sparrow, Field & Spizella pusilla & SPPU1 & FISP & 5630 & $\mathrm{R}$ & Ground, Shrub \\
\hline Sparrow, Five-striped & Amphispiza quinquestriata & AMQU & FSSP & 5742 & $\mathrm{R}$ & Shrub, Ground \\
\hline Sparrow, Fox & Passerella iliaca & PAIL & FOSP & 5850 & $\mathrm{~B}$ & Ground, Shrub \\
\hline Sparrow, Golden-crowned & Zonotrichia atricapilla & ZOAT & GCSP & 5570 & $\mathrm{R}$ & Ground, Woody Lower Canopy \\
\hline Sparrow, Grasshopper & Ammodramus savannarum & AMSA & GRSP & 5460 & $A$ & Ground \\
\hline Sparrow, Harris' & Zonotrichia querula & ZOQU & HASP & 5530 & $\mathrm{R}$ & Ground \\
\hline Sparrow, Henslow's & Ammodramus henslowii & AMHE & HESP & 5470 & $\mathrm{R}$ & Ground \\
\hline Sparrow, House & Passer domesticus & PADO1 & HOSP & 6882 & $\mathrm{R}$ & MMS, Woody Upper Canopy \\
\hline Sparrow, Lark & Chondestes grammacus & CHGR & LASP & 5520 & A & Ground, Shrub \\
\hline Sparrow, Le Conte's & Ammodramus leconteii & AMLE & LCSP & 5480 & $\mathrm{R}$ & Ground \\
\hline Sparrow, Lincoln's & Melospiza lincolnii & MELI & LISP & 5830 & A & Ground \\
\hline Sparrow, Olive & Arremonops rufivirgatus & ARRU & OLSP & 5860 & $\mathrm{R}$ & Shrub, Cactus \\
\hline Sparrow, Rufous-crowned & Aimophila ruficeps & AIRU & RCSP & 5800 & $\mathrm{R}$ & Ground, Shrub \\
\hline Sparrow, Rufous-winged & Aimophila carpalis & AICA & RWSP & 5790 & $\mathrm{R}$ & Shrub, Cactus \\
\hline Sparrow, Sage & Amphispiza belli & AMBE1 & SAGS & 5740 & $\mathrm{~B}$ & Shrub, Ground \\
\hline Sparrow, Savannah & Passerculus sandwichensis & PASA & SAVS & 5420 & $\mathrm{~B}$ & Ground \\
\hline Sparrow, Seaside & Ammodramus maritimus & AMMA3 & SESP & 5500 & $\mathrm{R}$ & Ground, Shrub \\
\hline Sparrow, Sharp-tailed & Ammodramus caudacutus & AMCA1 & STSP & 5490 & $\mathrm{R}$ & Ground, Shrub \\
\hline Sparrow, Song & Melospiza melodia & MEME & SOSP & 5810 & $\mathrm{~B}$ & Ground, Shrub \\
\hline Sparrow, Swamp & Melospiza georgiana & MEGE & SWSP & 5840 & $\mathrm{~B}$ & Shrub, Ground \\
\hline Sparrow, Vesper & Pooecetes gramineus & POGR1 & VESP & 5400 & $\mathrm{~B}$ & Ground \\
\hline Sparrow, White-crowned & Zonotrichia leucophrys & ZOLE & WCSP & 5540 & $\mathrm{~B}$ & Shrub, Ground \\
\hline Sparrow, White-throated & Zonotrichia albicollis & ZOAL & WTSP & 5580 & $\mathrm{~B}$ & Ground, Shrub \\
\hline Spoonbill, Roseate & Ajaia ajaja & AJAJ & ROSP & 1830 & $\mathrm{R}$ & Woody Lower Canopy, Shrub \\
\hline Starling, European & Sturnus vulgaris & STVU & EUST & 4930 & $\mathrm{R}$ & Woody Upper Canopy, MMS \\
\hline Stilt, Black-necked & Himantopus mexicanus & HIME & BNST & 2260 & $\mathrm{R}$ & Ground \\
\hline Stork, Wood & Mycteria americana & MYAM & WOST & 1880 & $\mathrm{R}$ & Woody Upper Canopy \\
\hline Surfbird & Aphriza virgata & APVI & SURF & 2820 & $\mathrm{R}$ & Ground \\
\hline Swallow, Bank & Riparia riparia & RIRI & BANS & 6160 & $A$ & Bank \\
\hline Swallow, Barn & Hirundo rustica & HIRU & BARS & 6130 & A & MMS \\
\hline Swallow, Cave & Hirundo fulva & HIFU & CASW & 6121 & C & Cave, MMS \\
\hline Swallow, Cliff & Hirundo pyrrhonota & HIPY & CLSW & 6120 & A & MMS, Cliff \\
\hline $\begin{array}{l}\text { Swallow, Northern Rough- } \\
\text { winged }\end{array}$ & Stelgidopteryx serripennis & STSE & NRWS & 6170 & $A$ & Bank, Cliff \\
\hline Swallow, Tree & Tachycineta bicolor & TABI & TRES & 6140 & $\mathrm{~B}$ & Snag, Woody Upper Canopy \\
\hline Swallow, Violet-green & Tachycineta thalassina & TATH & VGSW & 6150 & $A$ & Snag, Woody Upper Canopy \\
\hline Swan, Mute & Cygnus olor & CYOL & MUSW & 1782 & $\mathrm{R}$ & Ground \\
\hline Swan, Trumpeter & Cygnus buccinator & CYBU & TRUS & 1810 & $\mathrm{R}$ & Ground \\
\hline Swan, Whistling & Cygnus columbianus & CYCO1 & WHSW & 1800 & $\mathrm{R}$ & Ground \\
\hline Swan, Whooper & Cygnus cygnus & CYCY & WHOS & 1790 & $\mathrm{R}$ & Ground \\
\hline Swift, Black & Cypseloides niger & CYNI & BLSW & 4220 & $A$ & Cliff \\
\hline Swift, Chimney & Chaetura pelagica & CHPE & CHSW & 4230 & $A$ & MMS, Woody Upper Canopy \\
\hline Swift, Vaux's & Chaetura vauxi & CHVA & VASW & 4240 & $A$ & Snag, MMS \\
\hline Swift, White-throated & Aeronautes saxatalis & AESA & WTSW & 4250 & $A$ & Cliff \\
\hline Tanager, Hepatic & Piranga flava & PIFL & HETA & 6090 & $A$ & Woody Upper Canopy \\
\hline Tanager, Scarlet & Piranga olivacea & PIOL & SCTA & 6080 & $A$ & Woody Upper Canopy \\
\hline Tanager, Summer & Piranga rubra & PIRU & SUTA & 6100 & $A$ & Woody Upper Canopy \\
\hline Tanager, Western & Piranga ludoviciana & PILU & WETA & 6070 & $A$ & Woody Upper Canopy \\
\hline
\end{tabular}




\begin{tabular}{|c|c|c|c|c|c|}
\hline $\begin{array}{l}\text { LCTA } \\
\text { CODE }\end{array}$ & NEST TYPE & FOOD TYPE & $\begin{array}{l}\text { FORAGING } \\
\text { SUBSTRATE }\end{array}$ & FORAGING TECHNIQUE & GE NERAL HABITAT \\
\hline PAMO1 & Cavity & Seeds, Insects & Ground & Ground Gleaner & Forest, Urban \\
\hline SPPU1 & Cup & Insects, Seeds & Ground, Foliage & Ground Gleaner, Foliage Gleaner & Shrubland, Forest Edge \\
\hline AMQU & Cup & Insects, Seeds & Ground, Foliage & Ground Gleaner, Foliage Gleaner & Shrubland, Grassland \\
\hline PAIL & Cup & Insects, Seeds & Ground & Ground Gleaner & Forest, Forest Edge \\
\hline ZOAT & Cup & Insects, Seeds & Ground & Ground Gleaner & Shrubland \\
\hline AMSA & Cup & Insects, Seeds & Ground & Ground Gleaner & Grassland, Cropland \\
\hline ZOQU & Cup & Insects, Seeds & Ground, Foliage & Ground Gleaner, Foliage Gleaner & Shrubland, Forest \\
\hline AMHE & Cup & Insects, Seeds & Ground & Ground Gleaner & Grassland, Shrubland \\
\hline PADO1 & Cavity, Sphere & Seeds, Insects & Ground, Foliage & Ground Gleaner, Foliage Gleaner & Urban, Cropland \\
\hline CHGR & Cup & Seeds, Insects & Ground & Ground Gleaner & Grassland, Shrubland \\
\hline AMLE & Cup & Insects, Seeds & Ground & Ground Gleaner & Swamp/Marsh \\
\hline MELI & Cup & Insects, Seeds & Ground & Ground Gleaner & Swamp/Marsh, Riparian \\
\hline ARRU & Sphere & Insects, Seeds & Ground & Ground Gleaner & Shrubland, Riparian \\
\hline AIRU & Cup & Insects, Seeds & Ground, Foliage & Ground Gleaner, Foliage Gleaner & Shrubland, Grassland \\
\hline AICA & Cup & Insects, Seeds & Foliage, Air & Foliage Gleaner, Hawker & Shrubland, Grassland \\
\hline AMBE1 & Cup & Insects, Seeds & Ground, Foliage & Ground Gleaner, Foliage Gleaner & Shrubland \\
\hline PASA & Cup & Insects, Seeds & Ground & Ground Gleaner & Grassland, Swamp/Marsh \\
\hline AMMA3 & Cup & Insects, Seeds & Ground, Foliage & Ground Gleaner, Foliage Gleaner & Swamp/Marsh \\
\hline AMCA1 & Cup & Insects, Seeds & Ground & Ground Gleaner & Swamp/Marsh \\
\hline MEME & Cup & Insects, Seeds & Ground, Foliage & Ground Gleaner, Foliage Gleaner & Riparian, Forest Edge \\
\hline MEGE & Cup & Insects, Seeds & Ground & Ground Gleaner & Swamp/Marsh \\
\hline POGR1 & Cup & Insects, Seeds & Ground & Ground Gleaner & Grassland, Shrubland \\
\hline ZOLE & Cup & Insects, Seeds & Ground, Foliage & Ground Gleaner, Foliage Gleaner & Shrubland, Urban \\
\hline ZOAL & Cup & Insects, Seeds & Ground, Foliage & Ground Gleaner, Foliage Gleaner & Forest, Forest Edge \\
\hline AJAJ & Platform & Fish, Aquatic Inverts & Water & Prober & Swamp/Marsh, Freshwater \\
\hline STVU & Cavity & Insects, Fruit & Ground, Foliage & Ground Gleaner, Foliage Gleaner & Grassland, Urban \\
\hline HIME & Scrape & Aquatic Inverts, Insects & Water, Ground & Prober, Ground Gleaner & Swamp/Marsh, Freshwater \\
\hline MYAM & Platform & Fish, Aquatic Inverts & Water & Prober & Swamp/Marsh, Riparian \\
\hline APVI & Scrape & Insects, Aquatic Inverts & Ground, Water & Ground Gleaner, Prober & Coastal \\
\hline RIRI & Burrow & Insects & Air & Aerial Foraging & Freshwater, Grassland \\
\hline HIRU & Cup & Insects, Fruit & Air & Aerial Foraging & Freshwater, Grassland \\
\hline HIFU & Cup & Insects & Air & Aerial Foraging & Freshwater, Grassland \\
\hline HIPY & Cup & Insects, Fruit & Air & Aerial Foraging & Freshwater, Grassland \\
\hline STSE & Burrow, Crevice & Insects & Air & Aerial Foraging & Freshwater, Grassland \\
\hline TABI & Cavity & Insects, Fruit & Air, Foliage & Aerial Foraging, Foliage Gleaner & Freshwater, Grassland \\
\hline TATH & Cavity & Insects & Air & Aerial Foraging & Forest \\
\hline CYOL & Platform & Greens, Aquatic Inverts & Water & Surface Dips, Dabbler & Freshwater, Swamp/Marsh \\
\hline CYBU & Platform & Greens, Aquatic Inverts & Water & Surface Dips, Dabbler & Freshwater, Swamp/Marsh \\
\hline CYCO1 & Platform & Greens, Aquatic Inverts & Water & Surface Dips, Dabbler & Freshwater, Tundra \\
\hline CYCY & Platform & Greens, Aquatic Inverts & Water & Surface Dips, Dabbler & Coastal, Tundra \\
\hline CYNI & Saucer & Insects & Air & Aerial Foraging & Rocky Slope, Shoreline \\
\hline CHPE & Saucer & Insects & Air & Aerial Foraging & Forest, Urban \\
\hline CHVA & Saucer & Insects & Air & Aerial Foraging & Forest \\
\hline AESA & Crevice & Insects & Air & Aerial Foraging & Rocky Slope, Shoreline \\
\hline PIFL & Saucer & Insects, Fruit & Foliage, Air & Foliage Gleaner, Hawker & Forest, Riparian \\
\hline PIOL & Saucer & Insects, Fruit & Air, Foliage & Hover Gleaner, Foliage Gleaner & Forest \\
\hline PIRU & Cup & Insects, Fruit & Foliage, Air & Foliage Gleaner, Hawker & Forest, Riparian \\
\hline PILU & Cup & Insects, Fruit & Foliage, Air & Foliage Gleaner, Hawker & Forest \\
\hline
\end{tabular}




\begin{tabular}{|c|c|c|c|c|c|c|}
\hline COMMON NAME & SCIENTIFIC NAME & $\begin{array}{l}\text { LCTA } \\
\text { CODE }\end{array}$ & $\begin{array}{l}\text { ALPHA } \\
\text { CODE }\end{array}$ & AOU\# & $\begin{array}{l}\text { NEO } \\
\text { STAT }\end{array}$ & NEST LOCATION \\
\hline Tattler, Wandering & Heteroscelus incanus & HEIN & WATA & 2590 & $\mathrm{R}$ & Ground \\
\hline Teal, Blue-winged & Anas discors & ANDI & BWTE & 1400 & $\mathrm{R}$ & Ground \\
\hline Teal, Cinnamon & Anas cyanoptera & ANCY & CITE & 1410 & $\mathrm{R}$ & Ground \\
\hline Teal, Green-winged & Anas crecca & ANCR & AGWT & 1390 & $\mathrm{R}$ & Ground \\
\hline Tern, Aleutian & Sterna aleutica & STAL1 & ALTE & 0730 & $\mathrm{R}$ & Ground \\
\hline Tern, Arctic & Sterna paradisaea & STPA1 & ARTE & 0710 & $\mathrm{R}$ & Ground \\
\hline Tern, Black & Chlidonias niger & $\mathrm{CHNI}$ & BLTE & 0770 & $\mathrm{R}$ & Floating, Ground \\
\hline Tern, Caspian & Sterna caspia & STCA1 & CATE & 0640 & $\mathrm{R}$ & Ground \\
\hline Tern, Common & Sterna hirundo & STHI & COTE & 0700 & $\mathrm{R}$ & Ground \\
\hline Tern, Elegant & Sterna elegans & STEL & ELTE & 0660 & $\mathrm{R}$ & Ground \\
\hline Tern, Forster's & Sterna forsteri & STFO & FOTE & 0690 & $\mathrm{R}$ & Floating, Ground \\
\hline Tern, Gull-billed & Sterna nilotica & STNI & GBTE & 0630 & $\mathrm{R}$ & Ground, Floating \\
\hline Tern, Least & Sterna antillarum & STAN1 & LETE & 0740 & $\mathrm{R}$ & Ground \\
\hline Tern, Roseate & Sterna dougallii & STDO & ROST & 0720 & $\mathrm{R}$ & Ground \\
\hline Tern, Royal & Sterna maxima & STMA1 & ROYT & 0650 & $\mathrm{R}$ & Ground \\
\hline Tern, Sandwich & Sterna sandvicensis & STSA & SATE & 0670 & $\mathrm{R}$ & Ground \\
\hline Tern, Sooty & Sterna fuscata & STFU & SOTE & 0750 & $\mathrm{R}$ & Ground \\
\hline Thrasher, Bendire's & Toxostoma bendirei & TOBE & BETH & 7080 & B & Shrub, Woody Lower Canopy \\
\hline Thrasher, Brown & Toxostoma rufum & TORU & BRTH & 7050 & $\mathrm{R}$ & Shrub, Woody Lower Canopy \\
\hline Thrasher, California & Toxostoma redivivum & TORE & CATH & 7100 & $\mathrm{R}$ & Shrub, Woody Lower Canopy \\
\hline Thrasher, Crissal & Toxostoma dorsale & TODO & CRTH & 7120 & $\mathrm{R}$ & Shrub, Woody Lower Canopy \\
\hline Thrasher, Curve-billed & Toxostoma curvirostre & TOCU & CBTH & 7070 & $\mathrm{R}$ & Shrub, Woody Lower Canopy \\
\hline Thrasher, Le Conte's & Toxostoma lecontei & TOLE & LCTH & 7110 & $\mathrm{R}$ & Shrub, Woody Lower Canopy \\
\hline Thrasher, Long-billed & Toxostoma longirostre & TOLO & LBTH & 7060 & $\mathrm{R}$ & Shrub, Woody Lower Canopy \\
\hline Thrasher, Sage & Oreoscoptes montanus & ORMO & SATH & 7020 & B & Shrub, Ground \\
\hline Thrush, Gray-cheeked & Catharus minimus & CAMI2 & GCTH & 7570 & $A$ & Shrub, Woody Lower Canopy \\
\hline Thrush, Hermit & Catharus guttatus & CAGU & HETH & 7590 & $\mathrm{~B}$ & Ground, Woody Lower Canopy \\
\hline Thrush, Swainson's & Catharus ustulatus & CAUS & SWTH & 7580 & $A$ & Shrub, Woody Lower Canopy \\
\hline Thrush, Varied & Ixoreus naevius & IXNA & VATH & 7630 & $\mathrm{R}$ & Woody Lower Canopy \\
\hline Thrush, Wood & Hylocichla mustelina & HYMU & WOTH & 7550 & $A$ & Woody Upper Canopy \\
\hline Tit, Siberian & Parus cinctus & $\mathrm{PACl}$ & SITI & 7390 & $\mathrm{R}$ & Snag, Woody Lower Canopy \\
\hline Titmouse, Bridled & Parus wollweberi & PAWO & $\mathrm{BRTI}$ & 7340 & $\mathrm{R}$ & Woody Lower Canopy, Snag \\
\hline Titmouse, Plain & Parus inornatus & PAIN & PLTI & 7330 & $\mathrm{R}$ & Woody Lower Canopy, Snag \\
\hline Titmouse, Tufted & Parus bicolor & PABI & TUTI* & 7310 & $\mathrm{R}$ & Woody Upper Canopy, Snag \\
\hline Towhee, Abert's & Pipilo aberti & PIAB & ABTO & 5920 & $\mathrm{R}$ & Shrub, Woody Lower Canopy \\
\hline Towhee, Brown & Pipilo fuscus & PIFU & BRTO & 5910 & $\mathrm{R}$ & Shrub, Woody Lower Canopy \\
\hline Towhee, Green-tailed & Pipilo chlorurus & $\mathrm{PICH}$ & GTTO & 5900 & $A$ & Shrub, Ground \\
\hline Towhee, Rufous-sided & Pipilo erythrophthalmus & PIER & RSTO & 5870 & $B$ & Ground, Shrub \\
\hline Trogon, Elegant & Trogon elegans & TREL & ELTR & 3890 & $\mathrm{C}$ & Snag, Bank \\
\hline Tropicbird, Red-billed & Phaethon aethereus & PHAE & RBTR & 1130 & $\mathrm{R}$ & Ground, Cliff \\
\hline Tropicbird, White-tailed & Phaethon lepturus & PHLE & WTTR & 1120 & $\mathrm{R}$ & Ground, Cliff \\
\hline Turkey, Wild & Meleagris gallopavo & MEGA & WITU* & 3100 & $\mathrm{R}$ & Ground \\
\hline Turnstone, Black & Arenaria melanocephala & ARME & BLTU & 2840 & $\mathrm{R}$ & Ground \\
\hline Turnstone, Ruddy & Arenaria interpres & ARIN & RUTU & 2830 & $\mathrm{R}$ & Ground \\
\hline $\begin{array}{l}\text { Tyranulet, } \\
\text { Beardless }\end{array}$ & Camptostoma imberbe & CAIM & NBTY & 4720 & C & Woody Upper Canopy \\
\hline Veery & Catharus fuscescens & CAFU2 & VEER & 7560 & A & Ground, Shrub \\
\hline Verdin & Auriparus flaviceps & AUFL & VERD & 7460 & $\mathrm{R}$ & Shrub, Cactus \\
\hline Vireo, Bell's & Vireo bellii & VIBE & BEVI & 6330 & $A$ & Shrub, Woody Lower Canopy \\
\hline
\end{tabular}




\begin{tabular}{|c|c|c|c|c|c|}
\hline $\begin{array}{l}\text { LCTA } \\
\text { CODE }\end{array}$ & NEST TYPE & FOOD TYPE & $\begin{array}{l}\text { FORAGING } \\
\text { SUBSTRATE }\end{array}$ & FORAGING TECHNIQUE & GENERAL HABITAT \\
\hline HEIN & Scrape & Insects, Aquatic Inverts & Water & Prober & Freshwater, Riparian \\
\hline ANDI & Scrape & Seeds, Greens & Water & Surface Dips, Dabbler & Freshwater, Swamp/Marsh \\
\hline ANCY & Scrape & Seeds, Insects & Water & Surface Dips, Dabbler & Swamp/Marsh, Freshwater \\
\hline ANCR & Scrape & Seeds, Aquatic Inverts & Ground, Water & Ground Gleaner, Dabbler & Swamp/Marsh, Freshwater \\
\hline STAL1 & Scrape & Fish, Aquatic Inverts & Water & High Dives & Coastal, Pelagic \\
\hline $\mathrm{CHNI}$ & Platform, Scrape & Insects, Aquatic Inverts & Air, Water & Hawker, Skimmer & Swamp/Marsh \\
\hline STCA1 & Scrape & Fish, Aquatic Inverts & Water & High Dives & Coastal, Freshwater \\
\hline STHI & Scrape & Fish, Aquatic Inverts & Water & High Dives & Coastal, Tundra \\
\hline STEL & Scrape & Fish & Water & Scavenger, High Dives & Coastal, Pelagic \\
\hline STFO & Platform, Saucer & Fish, Insects & Water, Air & High Dives, Aerial Foraging & Swamp/Marsh \\
\hline STMA1 & Scrape & Fish, Aquatic Inverts & Water & High Dives, Skimmer & Coastal \\
\hline STSA & Scrape & Fish, Aquatic Inverts & Water & High Dives, Skimmer & Coastal \\
\hline STFU & Scrape & Fish, Aquatic Inverts & Water & Hover \& Pick & Coastal, Freshwater \\
\hline TOBE & Cup & Insects, Fruit & Ground, Foliage & Ground Gleaner, Foliage Gleaner & Shrubland \\
\hline TORU & Cup & Insects, Small Verts & Ground, Foliage & Ground Gleaner, Foliage Gleaner & Shrubland, Forest Edge \\
\hline TORE & Cup & Insects, Fruit & Ground, Foliage & Digging, Foliage Gleaner & Shrubland, Riparian \\
\hline TODO & Cup & Insects, Fruit & Ground & Digging & Shrubland, Riparian \\
\hline TOCU & Cup & Insects, Fruit & Ground, Foliage & Ground Gleaner, Foliage Gleaner & Shrubland \\
\hline TOLE & Cup & Insects, Small Verts & Ground, Foliage & Digging, Foliage Gleaner & Shrubland \\
\hline TOLO & Cup & Insects, Small Verts & Ground, Foliage & Ground Gleaner, Foliage Gleaner & Shrubland, Riparian \\
\hline $\mathrm{PACl}$ & Cavity & Insects, Seeds & Foliage, Bark & Foliage Gleaner, Bark Gleaner & Forest, Riparian \\
\hline PAWO & Cavity & Insects & Foliage, Bark & Foliage Gleaner, Bark Gleaner & Forest, Shrubland \\
\hline PAIN & Cavity & Insects, Fruit & Foliage, Bark & Foliage Gleaner, Bark Gleaner & Forest \\
\hline PABI & Cavity & Insects, Seeds & Foliage, Bark & Foliage Gleaner, Bark Gleaner & Forest, Shrubland \\
\hline PIAB & Cup & Seeds, Insects & Ground & Ground Gleaner & Shrubland, Riparian \\
\hline PIFU & Cup & Seeds, Insects & Ground & Ground Gleaner & Shrubland, Riparian \\
\hline $\mathrm{PICH}$ & Cup & Insects, Seeds & Ground & Ground Gleaner & Shrubland, Riparian \\
\hline PIER & Cup & Insects, Seeds & Ground, Foliage & Ground Gleaner, Foliage Gleaner & Forest Edge, Shrubland \\
\hline TREL & Cavity & Insects, Fruit & Air & Hawker, Hover Gleaner & Forest, Shrubland \\
\hline PHAE & Burrow, Crevice & Fish, Aquatic Inverts & Water & High Dives & Coastal, Pelagic \\
\hline PHLE & Burrow, Crevice & Fish, Aquatic Inverts & Water & High Dives & Coastal, Pelagic \\
\hline MEGA & Scrape & Seeds, Insects & Ground & Ground Gleaner & Forest, Riparian \\
\hline ARME & Scrape & Aquatic Inverts, Fruit & Ground & Ground Gleaner & Shoreline, Tundra \\
\hline ARIN & Scrape & Insects, Aquatic Inverts & Ground, Water & Ground Gleaner, Prober & Coastal, Tundra \\
\hline CAIM & Sphere & Insects, Fruit & Air & Hawker & Riparian, Forest \\
\hline CAFU2 & Cup & Insects, Fruit & Ground & Ground Gleaner, Swooper & Forest \\
\hline AUFL & Sphere & Insects, Fruit & Foliage, Bark & Foliage Gleaner, Bark Gleaner & Shrubland \\
\hline VIBE & Cup & Insects, Fruit & Foliage & Foliage Gleaner & Riparian, Shrubland \\
\hline
\end{tabular}




\begin{tabular}{|c|c|c|c|c|c|c|}
\hline COMMON NAME & SCIENTIFIC NAME & $\begin{array}{l}\text { LCTA } \\
\text { CODE }\end{array}$ & $\begin{array}{l}\text { ALPHA } \\
\text { CODE }\end{array}$ & AOU\# & $\begin{array}{l}\text { NEO } \\
\text { STAT }\end{array}$ & NEST LOCATION \\
\hline Vireo, Black-capped & Vireo atricapillus & VIAT & BCVI & 6300 & A & Woody Lower Canopy \\
\hline Vireo, Gray & Vireo vicinior & VIVI & GRVI & 6340 & A & Shrub, Woody Lower Canopy \\
\hline Vireo, Hutton's & Vireo huttoni & VIHU & HUVI & 6320 & $\mathrm{R}$ & Woody Lower Canopy \\
\hline Vireo, Philadelphia & Vireo philadelphicus & VIPH & PHVI & 6260 & A & Woody Upper Canopy \\
\hline Vireo, Solitary & Vireo solitarius & VISO & SOVI & 6290 & A & Woody Lower Canopy \\
\hline Vireo, Warbling & Vireo gilvus & VIGI & WAVI & 6270 & A & Woody Upper Canopy, Shrub \\
\hline Vireo, White-eyed & Vireo griseus & VIGR & WEVI & 6310 & A & Shrub, Woody Lower Canopy \\
\hline Vireo, Yellow-throated & Vireo flavifrons & VIFL & YTVI & 6280 & A & Woody Upper Canopy \\
\hline Vulture, Black & Coragyps atratus & COAT & BLVU & 3260 & $\mathrm{R}$ & Cliff, Ground \\
\hline Warbler, Arctic & Phylloscopus borealis & $\mathrm{PHBO}$ & ARWA & 7470 & $\mathrm{R}$ & Ground \\
\hline Warbler, Bachman's & Vermivora bachmanii & VEBA & BAWA & 6400 & $A$ & Vine Tangle, Woody Lower Canopy \\
\hline Warbler, Bay-breasted & Dendroica castanea & DECA2 & BBWA & 6600 & $A$ & Woody Upper Canopy \\
\hline Warbler, Black-and-white & Mniotilta varia & MNVA & BAWW & 6360 & $A$ & Ground, Woody Lower Canopy \\
\hline Warbler, Black-throated Blue & Dendroica caerulescens & DECA1 & BTBW & 6540 & $A$ & Shrub, Woody Lower Canopy \\
\hline Warbler, Black-throated Gray & Dendroica nigrescens & DENI & BTYW & 6650 & $A$ & Woody Upper Canopy \\
\hline $\begin{array}{l}\text { Warbler, Black-throated } \\
\text { Green }\end{array}$ & Dendroica virens & DEVI & BTNW & 6670 & $A$ & Woody Upper Canopy \\
\hline Warbler, Blackburnian & Dendroica fusca & DEFU & BLBW & 6620 & A & Woody Upper Canopy \\
\hline Warbler, Blackpoll & Dendroica striata & DEST & BLPW & 6610 & A & Woody Lower Canopy \\
\hline Warbler, Golden-cheeked & Dendroica chrysoparia & $\mathrm{DECH}$ & GCWA & 6660 & A & Woody Lower Canopy \\
\hline Warbler, Golden-winged & Vermivora chrysoptera & VECH & GWWA & 6420 & A & Ground \\
\hline Warbler, Grace's & Dendroica graciae & DEGR & GRWA & 6640 & A & Woody Upper Canopy \\
\hline Warbler, Hermit & Dendroica occidentalis & DEOC & HEWA & 6690 & A & Woody Upper Canopy \\
\hline Warbler, Hooded & Wilsonia citrina & $\mathrm{WICl}$ & HOWA & 6840 & A & Shrub, Woody Lower Canopy \\
\hline Warbler, Kentucky & Oporornis formosus & OPFO & KEWA & 6770 & A & Ground, Shrub \\
\hline Warbler, Kirtland's & Dendroica kirtlandii & DEKI & KIWA & 6700 & A & Ground \\
\hline Warbler, Lucy's & Vermivora luciae & VELU & LUWA & 6430 & $\mathrm{C}$ & Snag, Woody Lower Canopy \\
\hline Warbler, MacGillivray's & Oporornis tolmiei & ОРТО & MGWA & 6800 & A & Shrub, Woody Lower Canopy \\
\hline Warbler, Magnolia & Dendroica magnolia & DEMA & MAWA & 6570 & A & Woody Lower Canopy, Shrub \\
\hline Warbler, Mourning & Oporornis philadelphia & OPPH & MOWA & 6790 & A & Ground, Shrub \\
\hline Warbler, Nashville & Vermivora ruficapilla & VERU & NAWA & 6450 & A & Ground \\
\hline Warbler, Olive & Peucedramus taeniatus & PETA & OLWA & 6510 & $\mathrm{C}$ & Woody Upper Canopy \\
\hline Warbler, Orange-crowned & Vermivora celata & VECE & OCWA & 6460 & $A$ & Ground, Woody Lower Canopy \\
\hline Warbler, Palm & Dendroica palmarum & DEPA & PAWA* & 6720 & A & Ground, Woody Lower Canopy \\
\hline Warbler, Pine & Dendroica pinus & DEPI & PIWA & 6710 & $\mathrm{R}$ & Woody Upper Canopy \\
\hline Warbler, Prairie & Dendroica discolor & DEDI & PRAW & 6730 & A & Shrub, Woody Lower Canopy \\
\hline Warbler, Prothonotary & Protonotaria citrea & $\mathrm{PRCl}$ & PROW & 6370 & A & Snag, Woody Lower Canopy \\
\hline
\end{tabular}




\begin{tabular}{|c|c|c|c|c|c|}
\hline $\begin{array}{l}\text { LCTA } \\
\text { CODE }\end{array}$ & NEST TYPE & FOOD TYPE & $\begin{array}{l}\text { FORAGING } \\
\text { SUBSTRATE }\end{array}$ & FORAGING TECHNIQUE & GE NERAL HABITAT \\
\hline VIAT & Cup & Insects, Seeds & Foliage & Foliage Gleaner & Forest, Shrubland \\
\hline VIVI & Cup & Insects & Foliage, Ground & Foliage Gleaner, Ground Gleaner & Shrubland, Forest \\
\hline VIHU & Cup & Insects, Fruit & Foliage & Foliage Gleaner & Forest \\
\hline VIPH & Cup & Insects, Fruit & Air, Foliage & Hover Gleaner, Foliage Gleaner & Forest \\
\hline VISO & Cup & Insects, Fruit & Foliage, Air & Foliage Gleaner, Hawker & Forest \\
\hline VIGI & Cup & Insects, Fruit & Foliage, Air & Foliage Gleaner, Hover Gleaner & Forest, Riparian \\
\hline VIGR & Cup & Insects, Fruit & Foliage & Foliage Gleaner & Shrubland, Riparian \\
\hline VIFL & Cup & Insects, Fruit & Foliage & Foliage Gleaner & Forest, Forest Edge \\
\hline COAT & None & Carrion, Small Verts & Ground & Scavenger, High Patrol & Grassland, Shrubland \\
\hline PHBO & Oven & Insects & Foliage, Air & Foliage Gleaner, Hover Gleaner & Forest, Shrubland \\
\hline VEBA & Cup & Insects & Foliage & Foliage Gleaner & Swamp/Marsh \\
\hline DECA2 & Cup & Insects, Fruit & Foliage, Air & Foliage Gleaner, Hawker & Forest, Shrubland \\
\hline MNVA & Cup & Insects & Bark, Air & Bark Gleaner, Hawker & Forest \\
\hline DECA1 & Cup & Insects, Seeds & Air, Foliage & Hover Gleaner, Foliage Gleaner & Forest \\
\hline DENI & Cup & Insects & Foliage, Air & Foliage Gleaner, Hover Gleaner & Forest, Shrubland \\
\hline DEVI & Cup & Insects & Foliage, Air & Foliage Gleaner, Hawker & Forest \\
\hline DEFU & Cup & Insects, Fruit & Foliage, Air & Foliage Gleaner, Hover Gleaner & Forest \\
\hline DEST & Cup & Insects, Fruit & Foliage, Bark & Foliage Gleaner, Bark Gleaner & Forest \\
\hline VEPI & Cup & Insects & Foliage & Foliage Gleaner & Shrubland, Riparian \\
\hline $\mathrm{DECH}$ & Cup & Insects & Foliage, Air & Foliage Gleaner, Hawker & Forest \\
\hline VECH & Cup & Insects & Foliage & Foliage Gleaner & Grassland, Shrubland \\
\hline DEGR & Cup & Insects & Foliage, Air & Foliage Gleaner, Hawker & Forest \\
\hline DEOC & Cup & Insects & Foliage, Air & Foliage Gleaner, Hover Gleaner & Forest \\
\hline $\mathrm{WICl}$ & Cup & Insects & Foliage, Air & Foliage Gleaner, Hawker & Forest, Riparian \\
\hline OPFO & Cup & Insects, Fruit & Ground, Foliage & Ground Gleaner, Foliage Gleaner & Forest \\
\hline DEKI & Cup & Insects, Tree Sap & Foliage, Ground & Foliage Gleaner, Ground Gleaner & Forest \\
\hline VELU & Cavity & Insects & Foliage, Air & Foliage Gleaner, Hawker & Shrubland, Forest \\
\hline OPTO & Cup & Insects & Foliage, Bark & Foliage Gleaner, Bark Gleaner & Riparian, Forest Edge \\
\hline DEMA & Cup & Insects, Nectar & Air, Bark & Hover Gleaner, Bark Gleaner & Forest \\
\hline $\mathrm{OPPH}$ & Cup & Insects & Foliage, Bark & Foliage Gleaner, Bark Gleaner & Forest, Swamp/Marsh \\
\hline VERU & Cup & Insects & Foliage, Ground & Foliage Gleaner, Ground Gleaner & Forest, Riparian \\
\hline PETA & Cup & Insects & Foliage, Bark & Foliage Gleaner, Bark Gleaner & Forest \\
\hline VECE & Cup & Insects, Fruit & Foliage & Foliage Gleaner & Forest, Riparian \\
\hline DEPA & Cup & Insects, Fruit & Ground, Foliage & Ground Gleaner, Foliage Gleaner & Forest \\
\hline DEPI & Cup & Insects, Seeds & Bark, Foliage & Bark Gleaner, Foliage Gleaner & Forest \\
\hline DEDI & Cup & Insects & Foliage, Air & Foliage Gleaner, Hawker & Shrubland, Forest Edge \\
\hline $\mathrm{PRCl}$ & Cavity & Insects, Aquatic Inverts & Bark, Foliage & Bark Gleaner, Foliage Gleaner & Swamp/Marsh, Riparian \\
\hline
\end{tabular}




\begin{tabular}{|c|c|c|c|c|c|c|}
\hline COMMON NAME & SCIENTIFIC NAME & $\begin{array}{l}\text { LCTA } \\
\text { CODE }\end{array}$ & $\begin{array}{l}\text { ALPHA } \\
\text { CODE }\end{array}$ & AOU\# & $\begin{array}{l}\text { NEO } \\
\text { STAT }\end{array}$ & NEST LOCATION \\
\hline Warbler, Red-faced & Cardellina rubrifrons & CARU2 & RFWA & 6900 & $\mathrm{C}$ & Ground \\
\hline Warbler, Rufous-capped & Basileuterus rufifrons & BARU & RCWA & 6921 & $\mathrm{R}$ & Ground \\
\hline Warbler, Swainson's & Limnothlypis swainsonii & LISW & SWWA & 6380 & A & Shrub, Woody Lower Canopy \\
\hline Warbler, Tennessee & Vermivora peregrina & VEPE & TEWA & 6470 & A & Ground \\
\hline Warbler, Townsend's & Dendroica townsendi & DETO & TOWA & 6680 & A & Woody Lower Canopy \\
\hline Warbler, Virginia's & Vermivora virginiae & VEVI & VIWA & 6440 & A & Ground \\
\hline Warbler, Wilson's & Wilsonia pusilla & WIPU & WIWA & 6850 & A & Ground, Vine Tangle \\
\hline Warbler, Worm-eating & Helmitheros vermivorus & HEVE & WEWA & 6390 & A & Ground \\
\hline Warbler, Yellow & Dendroica petechia & DEPE1 & YWAR & 6520 & A & Shrub, Woody Lower Canopy \\
\hline Warbler, Yellow-rumped & Dendroica coronata & DECO & YRWA* & 6550 & B & Woody Upper Canopy \\
\hline Warbler, Yellow-throated & Dendroica dominica & DEDO & YTWA & 6630 & A & Woody Upper Canopy \\
\hline Waterthrush, Louisiana & Seiurus motacilla & SEMO & LOWA & 6760 & A & Ground, Bank \\
\hline Waterthrush, Northern & Seiurus noveboracensis & SENO & NOWA & 6750 & A & Ground, Bank \\
\hline Waxwing, Bohemian & Bombycilla garrulus & BOGA & BOWA & 6180 & $\mathrm{R}$ & Woody Upper Canopy \\
\hline Waxwing, Cedar & Bombycilla cedrorum & BOCE & CEDW & 6190 & B & Woody Upper Canopy \\
\hline Wheatear, Northern & Oenanthe oenanthe & OEOE & NOWH & 7650 & $\mathrm{R}$ & Ground \\
\hline Whimbrel & Numenius phaeopus & $\mathrm{NUPH}$ & WHIM & 2650 & $\mathrm{R}$ & Ground \\
\hline Whip-poor-will & Caprimulgus vociferus & CAVO & WPWI & 4170 & A & Ground \\
\hline Wigeon, American & Anas americana & ANAM & AMWI & 1370 & $\mathrm{R}$ & Ground \\
\hline Wigeon, Eurasian & Anas penelope & ANPE & EUWI & 1360 & $\mathrm{R}$ & Ground \\
\hline Willet & Catoptrophorus semipalmatus & CASE & WILL & 2580 & $\mathrm{R}$ & Ground \\
\hline Woodcock, American & Scolopax minor & SCMI & AMWO & 2280 & $\mathrm{R}$ & Ground \\
\hline Woodpecker, Acorn & Melanerpes formicivorus & MEFO & ACWO & 4070 & $\mathrm{R}$ & Snag, Woody Upper Canopy \\
\hline Woodpecker, Black-backed & Picoides arcticus & PIAR & BBWO & 4000 & $\mathrm{R}$ & Snag \\
\hline Woodpecker, Downy & Picoides pubescens & PIPU & DOWO & 3940 & $\mathrm{R}$ & Snag, Woody Upper Canopy \\
\hline Woodpecker, Gila & Melanerpes uropygialis & MEUR & GIWO & 4110 & $\mathrm{R}$ & Cactus, Woody Upper Canopy \\
\hline Woodpecker, Golden-fronted & Melanerpes aurifrons & MEAU & GFWO & 4100 & $\mathrm{R}$ & Snag, Woody Upper Canopy \\
\hline Woodpecker, Hairy & Picoides villosus & PIVI & HAWO & 3930 & $\mathrm{R}$ & Woody Upper Canopy, Snag \\
\hline Woodpecker, Ivory-billed & Campephilus principalis & CAPR & IBWO & 3920 & $\mathrm{R}$ & Snag, Woody Upper Canopy \\
\hline Woodpecker, Ladder-backed & Picoides scalaris & PISC & LBWO & 3960 & $\mathrm{R}$ & Woody Lower Canopy, Shrub \\
\hline Woodpecker, Lewis' & Melanerpes lewis & MELE & LEWO & 4080 & B & Woody Upper Canopy, Snag \\
\hline Woodpecker, Nuttall's & Picoides nuttallii & PINU1 & NUWO & 3970 & $\mathrm{R}$ & Snag, Woody Upper Canopy \\
\hline Woodpecker, Pileated & Dryocopus pileatus & DRPI & PIWO & 4050 & $\mathrm{R}$ & Snag, Woody Upper Canopy \\
\hline Woodpecker, Red-bellied & Melanerpes carolinus & MECA & RBWO & 4090 & $\mathrm{R}$ & Snag, Woody Upper Canopy \\
\hline Woodpecker, Red-cockaded & Picoides borealis & PIBO & RCWO & 3950 & $\mathrm{R}$ & Woody Upper Canopy \\
\hline Woodpecker, Red-headed & Melanerpes erythrocephalus & MEER & RHWO & 4060 & $\mathrm{R}$ & Snag, Woody Upper Canopy \\
\hline Woodpecker, Strickland's & Picoides stricklandi & PIST & STWO & 3980 & $\mathrm{R}$ & Snag, Woody Upper Canopy \\
\hline Woodpecker, Three-toed & Picoides tridactylus & PITR & TTWO & 4010 & $\mathrm{R}$ & Snag, Woody Lower Canopy \\
\hline Woodpecker, White-headed & Picoides albolarvatus & PIAL & WHWO & 3990 & $\mathrm{R}$ & Snag, Woody Lower Canopy \\
\hline Wren, Bewick's & Thryomanes bewickii & THBE & BEWR & 7190 & $\mathrm{R}$ & Woody Lower Canopy, Snag \\
\hline Wren, Cactus & Campylorhynchus brunneicapillus & CABR & CACW & 7130 & $\mathrm{R}$ & Cactus, Woody Lower Canopy \\
\hline Wren, Canyon & Catherpes mexicanus & CAME3 & CANW & 7170 & $\mathrm{R}$ & Cliff \\
\hline Wren, Carolina & Thryothorus ludovicianus & THLU & CARW & 7180 & $\mathrm{R}$ & Woody Lower Canopy, Snag \\
\hline Wren, House & Troglodytes aedon & TRAE & HOWR & 7210 & A & Woody Lower Canopy, Snag \\
\hline Wren, Marsh & Cistothorus palustris & CIPA & MAWR & 7250 & B & Reed \\
\hline Wren, Sedge & Cistothorus platensis & CIPL & SEWR & 7240 & B & Ground \\
\hline Wren, Winter & Troglodytes troglodytes & TRTR & WIWR & 7220 & $\mathrm{R}$ & Snag, Woody Lower Canopy \\
\hline Wrentit & Chamaea fasciata & CHFA & WREN & 7420 & $\mathrm{R}$ & Shrub, Woody Lower Canopy \\
\hline
\end{tabular}




\begin{tabular}{|c|c|c|c|c|c|}
\hline $\begin{array}{l}\text { LCTA } \\
\text { CODE }\end{array}$ & NEST TYPE & FOOD TYPE & $\begin{array}{l}\text { FORAGING } \\
\text { SUBSTRATE }\end{array}$ & FORAGING TECHNIQUE & GE NERAL HABITAT \\
\hline CARU2 & Cup & Insects & Foliage, Air & Foliage Gleaner, Hawker & Forest \\
\hline LISW & Cup & Insects & Ground, Foliage & Ground Gleaner, Foliage Gleaner & Swamp/Marsh, Riparian \\
\hline VEPE & Cup & Insects, Fruit & Foliage & Foliage Gleaner & Swamp/Marsh, Forest Edge \\
\hline DETO & Cup & Insects, Seeds & Foliage, Air & Foliage Gleaner, Hawker & Forest \\
\hline WIPU & Cup & Insects, Fruit & Foliage, Air & Foliage Gleaner, Hover Gleaner & Shrubland, Riparian \\
\hline HEVE & Cup & Insects & Foliage, Bark & Foliage Gleaner, Bark Gleaner & Riparian, Forest \\
\hline DEPE1 & Cup & Insects, Fruit & Foliage, Bark & Foliage Gleaner, Bark Gleaner & Riparian, Shrubland \\
\hline DECO & Cup & Insects, Fruit & Foliage, Air & Foliage Gleaner, Hawker & Forest \\
\hline DEDO & Cup & Insects & Bark, Foliage & Bark Gleaner, Foliage Gleaner & Forest, Swamp/Marsh \\
\hline BOCE & Cup & Fruit, Insects & Foliage, Air & Foliage Gleaner, Hawker & Forest, Forest Edge \\
\hline OEOE & Crevice, Burrow & Insects, Seeds & Ground, Air & Ground Gleaner, Hawker & Tundra, Rocky Slope \\
\hline $\mathrm{NUPH}$ & Scrape & Insects, Fruit & Ground, Water & Ground Gleaner, Prober & Swamp/Marsh, Tundra \\
\hline CAVO & None & Insects & Air & Aerial Foraging & Forest \\
\hline ANAM & Scrape & Greens, Aquatic Inverts & Water & Dabbler & Swamp/Marsh, Freshwater \\
\hline ANPE & Scrape & Greens, Seeds & Water & Dabbler & Coastal, Pelagic \\
\hline CASE & Scrape & Insects, Aquatic Inverts & Water & Prober & Coastal, Pelagic \\
\hline SCMI & Scrape & Insects & Ground, Water & Prober & Swamp/Marsh, Riparian \\
\hline MEFO & Cavity & Insects, Nuts & Bark, Air & Bark Gleaner, Bark Gleaner & Forest \\
\hline PIAR & Cavity & Insects, Seeds & Bark & Bark Gleaner & Forest \\
\hline MELE & Cavity & Insects, Nuts & Air, Ground & Hawker, Ground Gleaner & Forest, Riparian \\
\hline PINU1 & Cavity & Insects, Fruit & Bark, Air & Bark Gleaner, Hawker & Forest, Riparian \\
\hline DRPI & Cavity & Insects, Fruit & Bark & Bark Gleaner & Forest, Urban \\
\hline MECA & Cavity & Insects, Nuts & Bark, Air & Bark Gleaner, Hawker & Forest, Riparian \\
\hline PIBO & Cavity & Insects, Fruit & Bark & Bark Gleaner & Forest \\
\hline MEER & Cavity & Insects, Fruit & Air, Ground & Hawker, Swooper & Forest, Urban \\
\hline PIST & Cavity & Insects, Fruit & Bark, Foliage & Bark Gleaner, Foliage Gleaner & Forest \\
\hline PITR & Cavity & Insects, Tree Sap & Bark & Bark Gleaner & Forest, Riparian \\
\hline PIAL & Cavity & Insects, Seeds & Bark, Ground & Bark Gleaner, Ground Gleaner & Forest \\
\hline THBE & Cavity & Insects & Ground, Foliage & Ground Gleaner, Foliage Gleaner & Forest, Shrubland \\
\hline CABR & Sphere & Insects, Small Verts & Ground, Foliage & Ground Gleaner, Foliage Gleaner & Desert, Shrubland \\
\hline CAME3 & Crevice & Insects, Small Verts & Ground, Foliage & Ground Gleaner, Foliage Gleaner & Rocky Slope, Urban \\
\hline THLU & Cavity & Insects & Ground, Foliage & Ground Gleaner, Foliage Gleaner & Forest, Urban \\
\hline TRAE & Cavity & Insects & Ground, Foliage & Ground Gleaner, Foliage Gleaner & Forest, Urban \\
\hline CIPA & Sphere & Insects, Aquatic Inverts & Ground, Foliage & Ground Gleaner, Foliage Gleaner & Swamp/Marsh \\
\hline CIPL & Sphere & Insects & Ground, Foliage & Ground Gleaner, Foliage Gleaner & Swamp/Marsh \\
\hline TRTR & Cavity & Insects & Ground, Foliage & Ground Gleaner, Foliage Gleaner & Forest, Riparian \\
\hline CHFA & Cup & Insects, Fruit & Foliage, Bark & Foliage Gleaner, Bark Gleaner & Shrubland, Urban \\
\hline
\end{tabular}




\begin{tabular}{llllllll}
\hline COMMON NAME & SCIENTIFIC NAME & LCTA & ALPHA & AOU\# & NEO & NEST LOCATION \\
& & CODE & CODE & & STAT & \\
\hline Yellowlegs, Greater & Tringa melanoleuca & TRME & GRYE & 2540 & R & Ground \\
Yellowlegs, Lesser & Tringa flavipes & TRFL & LEYE & 2550 & R & Ground \\
Yellowthroat, Common & Geothlypis trichas & GETR & COYE & 6810 & A & Shrub, Woody Lower Canopy \\
Yellowthroat, Gray-crowned & Geothlypis poliocephala & GEPO & GCYE & 6821 & R & Ground
\end{tabular}




\begin{tabular}{llllll}
\hline $\begin{array}{l}\text { LCTA } \\
\text { CODE }\end{array}$ & NEST TYPE & FOOD TYPE & FORAGING & FORAGING TECHNIQUE & GENERAL HABITAT \\
\hline TRME & Scrape & Fish, Insects & Water & Prober & Coastal, Freshwater \\
TRFL & Scrape & Insects, Aquatic Inverts & Water, Ground & Prober, Ground Gleaner & Tundra \\
GETR & Cup & Insects, Seeds & Foliage, Air & Foliage Gleaner, Hover Gleaner & Forest Edge, Grassland \\
GEPO & Cup & Insects, Fruit & Foliage, Ground & Foliage Gleaner, Ground Gleaner & Forest Edge, Forest
\end{tabular}




\section{USACERL DISTRIBUTION}

Chief of Engineers

ATTN: CEHEC-IM-LH (2)

ATTN: CEHEC-IM-LP (2)

ATTN: CECC-R

ATTN: CERD-L

Army Headquarters

ATTN: DAIM-ED-N (5)

ATTN: DAMO-TRO

ATTN: ANOP

ATTN: AMSTE-EQ

ATTN: SFIM-AEC-ECN

ATTN: ATIC-CTS

ATTN: FKEN-EN

ATTN: AEAGC-TD-MO

ATTN: EAGC-TD-RMD

ATTN: APOP-TR

ATTN: APEN-EV

US Army Europe

ATTN:AEAEN-ENVR 09114

100th Support Group 09114 ATTN:AETT-EN-DPW

CMTC Hohenfels 09173 ATTN: AETTH-DPW

FORSCOM

Fts Gillem\& McPherson 30330

ATTN: AFOP-TE

ATTN: AFOP-TSR

ATTN: AFPI-ENE

Installations:

Fort Indiantown Gap 17003 ATTN: AFKA-ZQ-DE-E

Fort AP Hill 22427 ATTN: ANAP-PWE ATTN: AFZM-FHE

Fort McPherson 30330

ATTN: AFZK-EH-E

Fort Riley 66442

ATTN: AFZN-DE-V-N

Fort Polk 71459

ATTN: AFZH-DE-EN

Fort Sam Houston 78234

ATTN: AFZG-DE-EM

Fort Lewis 98433

ATTN: AFZH-DE-Q

Fort Carson 80913

ATTN: AFZC-ECM-NR

Fort Bragg 28307

ATTN: AFZA-PW-DW

Fort Campbell 42223

ATTN: AFZB-DPW-E

Fort McCoy 54656

ATTN: AFZR-DE-E

Fort Pickett 23824

ATTN: AFZA-FP-E
Fort Stewart 31314

ATTN: AFZP-DEV

Fort Buchanan 00934 ATTN: AFZK-B-EHE

Fort Devens 01433

ATTN: AFZD-DEM

Fort Drum 13602

ATTN: AFZS-EH-E

Fort Irwin 92310

ATTN: AFZJ-EHE-EN

Fort Hood 76544

ATTN: AFZF-DE-ENV

Fort Hunter Liggett 93928

ATTN: AFZW-HE-DE

Yakima Tng Center 98901-5000

ATTN: AFZH-Y-ENR

Fort Dix 08640

ATTN: ATZD-EH

TRADOC

Fort Monroe 23651 ATTN: ATBO-FE

Installations:

Fort Lee 23801 ATTN: ATZM-PTS-T

Fort Jackson 29207 ATTN: ATZJ-PWN

Fort Gordon 30905 ATTN: ATZH-DIE

Fort Benning 31905 ATTN: ATZB-PWN

Fort McClellan 36205 ATTN: ATZN-EM

Fort Rucker 36362 ATTN: ATZQ-DPW-EN

Fort Leonard Wood 64573 ATTN: ATZT-DPW-EE

Fort Leavenworth 66027 ATTN: ATZL-GCE

Fort Bliss 79916 ATTN: ATZC-DOE

Carlisle Barracks 17013 ATTN: ATZE-DPW-E

Fort Eustis 23604 ATTN: ATZF-PWE

Fort Chaffee 72905 ATTN: ATZR-ZF

Fort Sill 73503 ATTN: ATZR-B

Fort Huachuca 85613 ATTN: ATZS-EHB

Fort Knox 40121 ATTN: ATZK-PWE

USAMC Instal \& Srvc Activity ATTN: AMXEN-M 61299

Rock Island Arsenal

ATTN: AMSMC-EHR

ATTN: SMCRI-PWB
White Sands Missile Range ATTN: STEWS-ES-E

US Army Dugway Proving Ground ATTN: STEDP-EPO-CP

US Army Yuma Proving Ground ATTN: STEYP-ES-E

Aberdeen Proving Ground ATTN: STEAP-SH-ER

National Guard Bureau 20310 ATTN: NGB-ARE ATTN: NGB-ARI ATTN: NGB-ARO-TS

Army National Guard Ft. Richardson, AK 99505-5800 Phoenix, AZ 85008-3495 Sacramento, CA 95826-9101 Boise, ID 83705-8095 Jackson, MS 39209

Camp Shelby, MS 39407-5500

Oklahoma City, OK 73111-4389

Draper, UT 84020-1776

Braggs, OK 74423

Reading, MA 01867-1999 Camp Edwards, MA 02542 5003

Richmond, VA 23219

Columbia, SC 29201

Eastover, SC 29244

St. Augustine, FL 32085

Starke, FL 32091

Indianapolis, IN 4624

Lansing, MI 48913

Little Falls, MN 56345

Jefferson, MO 65101

Camp Roberts, CA 93451

2200

Camp Robinson, AR 72118

US Military Academy 10996 ATTN: MAEN-EV ATTN: DOPS

US Army Research Laboratory ATTN: AMSRL-OP-SDFE

US Govt Printing Office 20401 ATTN: Rec Sec/Deposit Sec

Defense Technical Info Ctr 22304 ATTN: DTIC-FAB 